\title{
Abstracts from 2020 national congress of the Italian Society of Hypertension (SIIA)
}

(C) Italian Society of Hypertension 2020

Metabolic Aspects and Associated Risk Factors

SODIUM-GLUCOSE CO-TRANSPORTER-2 INHIBITORS (SGLT2I) ARE "HYBRID" DIURETICS THAT PROTECT FROM TARGET ORGAN DAMAGE AND CARDIOVASCULAR EVENTS: A NEW “WEAPON" FOR INTERNISTS AND CARDIOLOGISTS

Francesco Spannella ${ }^{1,2}$, Federico Giulietti ${ }^{1,2}$, Chiara Di Pentima ${ }^{1}$, Riccardo Sarzani ${ }^{1,2}$

1) Department of Clinical and Molecular Sciences, University "Politecnica delle Marche”,Ancona, Italy; 2) Internal Medicine and Geriatrics, IRCCS INRCA, Ancona, Italy

INTRODUCTION: Sodium-Glucose Co-Transporter-2 Inhibitors (SGLT2i) act on the proximal renal tubule and have been found to exert relevant cardiovascular benefits beyond the mere glycemic control in patients with diabetes. Their use have been associated to a reduction in both target organ damage and cardiovascular events, mainly heart failure and progression of renal disease.

METHODS: We performed an extensive review of the available literature aiming at describing the main pathophysiological mechanisms of SGLT2i that may have led to such positive findings in the randomized clinical trials performed with this drug class.

RESULTS: These "antidiabetic" drugs act as "hybrid" diuretics. Indeed, they promote both natriuresis and osmotic diuresis due to glycosuria, leading to a reduction in blood volume and blood pressure. This peculiar activity, not found in the other classic diuretics, is mainly derived by the stimulation of the renal tubuleglomerular feedback, leading to the release of adenosine by macula densa cells, resulting in a vasoconstriction of the afferent arteriole and a reduction of renin release. These mechanisms lead to lower intraglomerular pressure, preventing the hyperfiltration-mediated organ damage tipically found in diabetes, hypertension and obesity. In the CREDENCE trial, Canagliflozin led to a $30 \%$ reduction in both the progression of renal disease and cardiovascular deaths in patients with diabetes (but no renal disease) who were also taking renin-angiotensin-system inhibitors. Natriuresis and glycosuria lead to lower circulating blood volume and pressure, resulting in lower cardiac overload. Indeed, SGLT2i have been found to reduce both hospitalizations and cardiovascular deaths in patients with heart failure, regardless the diabetic condition, also improving both symptoms and cardiac remodeling.

CONCLUSIONS: The beneficial effects of SGLT2i, supported by multiple clinical data, suggest a more extensive use of these drugs also by internists, cardiologists and nefrologists.

\section{THE ASSOCIATION BETWEEN HYPERURICEMIA AND ARTERIAL STIFFNESS AND COGNITIVE IMPAIRMENT IS STILL CONFIRMED AT NORMAL-HIGH VALUES}

P. Nazzaro, A. Nardecchia, M. Contini, G. Schirosi, L. De Benedittis, G. Aceto, A.M. Papagni

Dept of Neurosciences, Unit of Hypertension "AM. Pirrelli", "Aldo Moro" University of Bari, Italy

INTRODUCTION: Hyperuricemia (HSUA $>6 \mathrm{mg} / \mathrm{dl}$ ) is associated to increased arterial stiffness and cognitive impairment but recent studies highlighted that normal-high values of serum uric acid (SUA) might be predictive of heart failure.

AIM: To discern if borderline values $(5.34<$ BSUA $<6$ $\mathrm{mg} / \mathrm{dl})$ might be characterized by reduced arterial compliance and cognitive deficit.

METHODS: After medical visit (off), 530 hypertensives $(135 \pm 11 / 85 \pm 5)$, treated by ACEi or ARB, with similar 
age, history and state of hypertension, divided in 292 normouricemic (NSUA: $<5.34 \mathrm{mg} / \mathrm{dl}$ ), 73 BSUA e 165 (HSUA) underwent ABPM, identification of metabolic syndrome factors (MSn), arterial tonometry for stiffness measurement (PWVcf) and assessment of cognitive impairment (CIS) by multiple choice (1-4) 18-item questionnaire investigating different neuropsychological functions.

RESULTS: Patients showed similar hypertensive state $(134 \pm 15 / 80 \pm 9$ vs $136 \pm 14 / 81 \pm 8$ vs $136 \pm 15 /$ $81 \pm 9$;n.s.), confirmed by ABPM, but other significant characteristics. $\left(\mathrm{m} \pm \mathrm{s}: \mathrm{d} .{ }^{*}: \mathrm{p}<.05, * *: \mathrm{p}<.01, * * *: \mathrm{p}<\right.$ .001 vs NSUA; ${ }^{\circ}: \mathrm{p}<.05,{ }^{\circ}: \mathrm{p}<.01,{ }^{\circ}$ : $\mathrm{p}<.001$ vs BSUA).

\begin{tabular}{|l|l|l|l|l|}
\hline pts/var & SUA & MSn & PWVcf & CIS \\
\hline NSUA & $3.9 \pm 0.8$ & $2.54 \pm 1.23$ & $10.1 \pm 4.3$ & $25,5 \pm 7.1$ \\
\hline BSUA & $5.4 \pm 0.2^{* * *}$ & $2.64 \pm 1.26$ & $11.4 \pm 5.1^{*}$ & $27.9 \pm 8.1^{*}$ \\
\hline HSUA & $7.3 \pm 1 * * \circ 00$ & $2.83 \pm 1.11^{* *}$ & $11.7 \pm 3.6^{* * *}$ & $29.2 \pm 10.1^{* * *}$ \\
\hline
\end{tabular}

Pearson test showed the association between SUA and PWV (.309**) and CIS (.143**)

CONCLUSIONS: The findings highlight that the association between hyperuricemia and arterial stiffness and cognitive impairment is still at borderline values of serum uric acid although a more favourable metabolic state.

\section{SUBENDOCARDIAL PERFUSION IMPAIRMENT IN PATIENTS WITH METABOLIC SYNDROME}

Giani A, Gasparini L, Bortolani A, Stefani M, Santuz B, Mazzali G, Zamboni M, Fantin F

Department of Medicine, Section of Geriatrics, University of Verona, AOUI Verona, Italy

INTRODUCTION: Metabolic Syndrome (MS) is strongly associated to vascular damage, increased arterial stiffness and impaired myocardial perfusion. Subendocardial Viability Ratio (SEVR) is a non-invasive estimation of myocardial workload, oxygen supply and perfusion.

AIM: To describe the relation between arterial stiffness, SEVR and cardio-metabolic risk factors.

METHODS: A cohort of 55 patients, aged $59.9 \pm 10.8$ years, formed the study population; 28 subjects $(50.9 \%)$ had MS. All patients underwent a comprehensive clinical evaluation; anthropometric variables and blood pressure were registered and a blood venous sample, to assess glico-lipid profile, was obtained. All subjects underwent applanation tonometry, which provided Pulse Pressure (PP), Augmentation Pressure (AP), Augmentation Index (AI) and SEVR values.
RESULTS: In the overall study population, SEVR showed negative relation to mean $(\mathrm{r}=-0.301 ; \mathrm{p}=0.026)$ and systolic (borderline relation, $\mathrm{r}=-0.257 ; \mathrm{p}=0.058$ ) arterial pressure. No significant association was found between SEVR and age nor metabolic parameters. Patients with MS presented lower level of SEVR $(p=0.012)$, even after adjusting for age, sex and mean arterial pressure (MAP, $\mathrm{p}=0.040$ ). Subdividing the study population by the number of MS components, SEVR values significantly decreased as the number of MS components increased ( $p$ for trend 0.005 ). In a logistic backward regression analysis, both MS and MAP resulted significant predictors of SEVR, accounting for $18 \%$ of variance.

CONCLUSIONS: Impaired subendocardial perfusion is described in patients with MS, and it may represent an important pathophysiological determinant of the increased cardiovascular risk. SEVR estimation has been proved to be a significant tool to assess cardio-metabolic risk.

\section{PROGNOSTIC IMPORTANCE OF LOW VISIT-TO- VISIT SYSTOLIC BLOOD PRESSURE VARIABILITY IN HIGH-RISK HYPERTENSION}

\author{
R. Del Pinto ${ }^{\text {A }}$, D. Pietropaoli ${ }^{\text {A }}$, M. Dobre ${ }^{\text {B }}$, C. Ferri ${ }^{\text {A }}$
}

${ }^{a}$ University of L'Aquila, Department of Life, Health, and Environmental Sciences, San Salvatore Hospital, L'Aquila, Italy; ${ }^{b}$ Department of Medicine, Case Western Reserve University, University Hospitals Cleveland Medical Center, Cleveland, Ohio

INTRODUCTION: Besides high blood pressure variability (BPV), low BPV was associated with adverse cardiovascular (CV) prognosis in selected high-risk patients (chronic heart failure, complicated myocardial infarction). AIM: We explored this issue in high-risk hypertensive individuals from the Systolic Blood Pressure Intervention Trial (SPRINT), using a nonlinear approach with systolic BPV (SBPV) as a continuous variable.

METHODS: Long-term SBPV (coefficient of variation, $\mathrm{CoV} \%$ ) was calculated on quarterly visits from randomization until the occurrence of an outcome, excluding titration period and patients with missing visits. The primary outcome was any fatal/nonfatal CV event; secondary outcomes were all-cause mortality and the composite of $\mathrm{CV}$ events and mortality. We used Cox proportional hazard models with penalized smoothing splines to shape the risk of outcomes against continuous values of SBPV (independent variable). Multivariate (treatment arm, pulse pressure, age $\geq 75$ years, gender, race, $\mathrm{CV}$ disease [CVD], chronic kidney disease [CKD], smoke, number of BP medications) hazard ratios (HR, 95\% CI) were calculated 
using the reference range derived from the nonlinear model. Sensitivity analysis based on propensity score matching (PSM) was performed.

RESULTS: The association of SBPV with fatal/nonfatal $\mathrm{CV}$ events was J-shaped (lowest risk for CoV 5-10\%), while that with all-cause mortality and the composite outcome was linear (lowest risk for $\mathrm{CoV}<7 \%$ and $10 \%$, respectively). After multivariate adjustment, only low SBPV $(\mathrm{CoV}<5 \%)$ was associated with $\mathrm{CV}$ events (HR $1.85,95 \%$ CI $1.24-2.75, \mathrm{p}=0.003)$, especially ischemic heart disease (HR 2.76, 95\% CI 1.55-4.91; p <0.001). High SBPV $(\mathrm{CoV}>10 \%)$ was associated with the risk of the composite outcome (HR: 1.35, 95\% CI 1.02-1.80; $\mathrm{p}=0.037$ ). PSM, matching 566 non-smoker SPRINT participants without baseline CVD or CKD and having SBPV 5-10\% with an equal number of patients having different SBPV, confirmed the inverse association of low SBPV $(<5 \%)$ with CV events (HR 3.33, 95\% CI $1.10-10.18, \mathrm{p}=0.035$ ).

CONCLUSIONS: Non-linear model indicates that low visit-to-visit SBPV has prognostic relevance in high-risk hypertensive individuals from SPRINT. Dedicated trials are needed to test these findings and their potential therapeutic implications.

\section{SEASONAL VARIATION IN ESTIMATED CARDIOVASCULAR RISK IN PATIENTS WITH TYPE 2 DIABETES}

Stefano Ciardullo **, Emanuele Muraca*, Rosa Cannistraci $^{* \ddagger}$, Francesca Zerbini*, Giuseppina Manzoni*, Silvia Perra*, Eleonora Bianconi*, Alice Oltolini*, Guido Grassi ${ }^{\circ}$, Giuseppe Mancia ${ }^{* \ddagger}$, Guido Lattuada*, Gianluca Perseghin ${ }^{* *}$

*Department of Medicine and Rehabilitation, Policlinico di Monza, Monza, Italy; ${ }^{\ddagger}$ School of Medicine and Surgery, University of Milano Bicocca, Milan, Italy; ${ }^{\circ}$ Clinica Medica, University of Milano Bicocca, Milan, Italy

INTRODUCTION: Seasonal variations in several risk factors for cardiovascular events (CVD) were described. AIM: To evaluate the impact of seasonal variations in blood pressure (BP), lipid profile and glycaemic control on estimated CVD risk in patients with type 2 diabetes (T2D). METHODS: Retrospective monocentric study of patients with T2D who were visited at least once in the winter period (20/11-28/2) and once in the summer period (20/510/9), less than 8 months apart, for which data related to systolic (S) BP, diastolic (D) BP, body mass index, glycosylated haemoglobin (HbA1c), total cholesterol, HDL cholesterol and smoking habit were available on both occasions. The 10-year CVD risk was calculated using the UKPDS risk engine and the ASCVD risk estimator.

RESULTS: 411 patients were included in the study (mean age 66 years, 239 (58.2\%) males). Significant within-patient differences between winter and summer were found for the absolute risk of events assessed with both calculators $(\Delta \mathrm{w}$-s UKPDS-CHD: $1.33 \%, \Delta \mathrm{w}$-s UKPDS-Stroke $0.84 \%, \Delta \mathrm{w}-\mathrm{s}$ ASCVD $2.21 \%$ ). The mean change in SBP $(6.7 \mathrm{mmHg})$ was the main responsible for the change in risk estimated with both the UKPDS-Stroke $(\mathrm{r} 2=0.43)$ and the ASCVD $(\mathrm{r} 2=0.50)$ scores, while the change in total cholesterol was the main determinant of the change in risk for the UKPDS-CHD $(\mathrm{r} 2=0.34)$. A significant correlation was identified between changes in temperature and changes in SBP $(\rho=0.130, p=0.008)$, but not in total cholesterol or HbA1c.

CONCLUSIONS: Seasonal variations in the classic CVD risk factors influence the risk estimated using validated calculators. In particular, changes in SBP have great influence on changes in stroke risk and changes in cholesterol on the risk for the composite CVD endpoint.

\section{SERUM URIC ACID AND KIDNEY DISEASE MEASURES INDEPENDENTLY PREDICT CARDIOVASCULAR AND TOTAL MORTALITY: THE URRAH (URIC ACID RIGHT FOR HEART HEALTH) STUDY}

Elisa Russo ${ }^{1}$, Giovanna Leoncini ${ }^{1}$, Francesca Viazzi ${ }^{1}$, Roberto Pontremoli ${ }^{1}$, Carlo M. Barbagallo ${ }^{2}$, Michele Bombelli $^{3}$, Edoardo Casiglia ${ }^{4}$, Arrigo F.G. Cicero ${ }^{5}$, Massimo Cirillo ${ }^{6}$, Pietro Cirillo ${ }^{7}$, Giovambattista Desideri $^{8}$, Claudio Ferri ${ }^{8}$, Ferruccio Galletti ${ }^{9}$, Loreto Gesualdo $^{7}$, Guido Grassi ${ }^{3}$, Cristina Giannattasio ${ }^{3}$, Guido Iaccarino ${ }^{9}$, Francesca Mallamaci ${ }^{10}$, Alessandro Maloberti ${ }^{3}$, Stefano Masi ${ }^{11}$, Alberto Mazza ${ }^{12}$, Maria L. Muiesan ${ }^{13}$, Pietro Nazzaro ${ }^{7}$, Paolo Palatini ${ }^{4}$, Gianfranco Parati ${ }^{3}$, Marcello Rattazzi ${ }^{4}$, Massimo Salvetti ${ }^{13}$, Valérie Tikhonoff ${ }^{4}$, Giuliano Tocci ${ }^{14}$, Andrea Ungar ${ }^{15}$, Giulia Rivasi ${ }^{15}$, Paolo Verdecchia ${ }^{16}$, Agostino Virdis ${ }^{11}$, Massimo Volpe ${ }^{14}$,\& Claudio Borghi $^{5}$ on behalf of the Working Group on Uric Acid and Cardiovascular Risk of the Italian Society of Hypertension

${ }^{1}$ Department of Internal Medicine, University of Genoa and Policlinico San Martino, Genoa; ${ }^{2}$ Biomedical Department of Internal Medicine and Specialistics, University of Palermo, Palermo; ${ }^{3}$ Clinica Medica, Department of Medicine and Surgery, University of Milano-Bicocca, Monza; ${ }^{4}$ Department of Medicine, University of Padua, Padua, Italy; ${ }^{5}$ Biomedical 
Department of Internal Medicine and Specialistics, University of Palermo, Palermo; ${ }^{6}$ Department of Medicine, University of Salerno, Salerno, Italy; 7 Department of Medical Basic Sciences, Neurosciences and Sense Organs, University of Bari Medical School, Bari $^{8}$ Università degli Studi dell'Aquila, L'Aquila, Italy; 9 Department of Public Health, "Federico II" University of Naples, Naples, Italy; ${ }^{10}$ CNR-IFC, Clinical Epidemiology of Renal Diseases and Hypertension, Reggio Cal Unit, Reggio Calabria; ${ }^{11}$ Department of Clinical and Experimental Medicine, University of Pisa, Pisa, Italy; 12 Department of Internal Medicine, Santa Maria della Misericordia General Hospital, AULSS 5 Polesana, Rovigo, Italy; ${ }^{13}$ Department of Clinical and Experimental Sciences, University of Brescia, Brescia; ${ }^{14}$ Department of Clinical and Molecular Medicine, University of Rome Sapienza, Sant'Andrea Hospital, Rome, Italy; 15 Department of Geriatric and Intensive Care Medicine, Careggi Hospital and University of Florence, Florence, Italy; ${ }^{16}$ Hospital S. Maria della Misericordia, Perugia, Italy

INTRODUCTION: Serum uric acid (SUA) may predict the onset and progression of kidney disease (CKD), and the occurrence of cardiovascular (CVM) and total mortality (TM). Nevertheless, it is unclear which is the accurate definition of hyperuricemia (HU) in presence of CKD and whether HU and renal impairment are independent risk factor for mortality.

AIM: To analyse the relationship between SUA and CKD components in causing mortality.

METHODS: We retrospectively investigated a total of 21,963 patients from the URRAH study database for whom data about CVM and TM were available. Hyperuricemia was defined on the basis of the outcome specific cut-off separately identified by ROC curves for patients with and without eGFR $<60 \mathrm{ml} / \mathrm{min}$. The primary endpoints were CVM composite of the occurrence of fatal heart failure and fatal cerebrovascular and cardiac events, and TM. We calculated the hazard ratios (HR) for eGFR, SUA, albuminuria, and their combination.

RESULTS: After a 9.8-year follow-up, 1063 (4.8\%) patients developed non-fatal cardiovascular events, 1582 (7.2\%) cardiovascular and $3130(14.2 \%)$ total mortality. The incidence of CVM and TM increased in parallel with eGFR strata and with SUA quartiles. During 215,618 person-years of follow-up, the incidence rate for CVM, stratified on the basis of eGFR ( $>90$, between 60 and 90 and $<60 \mathrm{ml} / \mathrm{min}$ ) was significantly higher in patients with HU and Albuminuria (3.8, 22.1 and 19.1, respectively) as compared to those with only one risk factor or none $(0.4$, 2.8 and 3.1, respectively). For each SUA increase of $1 \mathrm{mg} /$ $\mathrm{dl}$ the risk of CVM and TM increased by $10 \%$ even after adjustment for potential confounding factors including eGFR and the presence of albuminuria.

CONCLUSIONS: $\mathrm{HU}$ is a risk factor for CVM and TM additively to eGFR strata and albuminuria, in patients at $\mathrm{CV}$ risk

\section{RELATIONSHIPS BETWEEN ARTERIAL STIFFNESS, SARCOPENIA AND SARCOPENIC OBESITY}

\author{
A. Zampieri ${ }^{1}$, A. Varalta $^{1}$, G. Manzato ${ }^{1}$, E. Zoico ${ }^{1}$, \\ M. Facci ${ }^{1}$, A. Falceri ${ }^{1}$, S. Nicolussi Paolaz ${ }^{1}$, \\ M. Zamboni ${ }^{1}$, F. Fantin ${ }^{1}$ \\ ${ }^{1}$ Department of Geriatrics, University of Verona, Verona, \\ Italy
}

INTRODUCTION: Aging and body composition changes are associated with arterial stiffness and increased cardiovascular risk.

AIM: to evaluate the correlation between arterial stiffness indexes, sarcopenia and sarcopenic obesity in a group of patients aged between 66 and 88 years.

METHODS. The population was divided into 4 groups: obese, sarcopenic, sarcopenic obese and normal patients. Anthropometric indexes (weight, BMI, waist and hip circumference), body composition (BIA), physical strength (Hand Grip Strength Test), arterial blood pressure (SBP, DBP, MBP, PP), lipid profile, glucose, creatinine and blood count from fasting blood samples were assessed. Carotidfemoral pulse wave velocity (cfPWV), carotid-radial pulse wave velocity (crPWV) and central arterial blood pressure were measured using transcutaneous tonometry. Arterial stiffness was also evaluated by CAVI.

RESULTS: The population included 72 patients $(57 \%$ women, $n=41$ ) with a mean age of $78.65 \pm 9.96$ years and a mean BMI of $26.37 \pm 6.38 \mathrm{~kg} / \mathrm{m} 2$. There was a direct correlation between CAVI and age and an inverse correlation between CAVI and SMI (ASM/h2) in both sexes $(r=0.37, p=0.002)$. CAVI was significantly higher in sarcopenic than in obese patients $(\mathrm{p}=0.01)$. cfPWV was significantly higher in sarcopenic obese subjects than in the other groups. After adjustment for age, sex, MBP, LDL, triglycerides and creatinine, cfPWV was significantly higher in sarcopenic obese than in normal $(p=0.004)$ and obese patients $(\mathrm{p}=0.02)$.

CONCLUSION: This study shows an association between arterial stiffness indexes, obesity and sarcopenic obesity in a population of elderly people, in particular a correlation between CAVI values and sarcopenia and between cfPWV and sarcopenic obesity in both sexes. Thus, in obese and 
sarcopenic obese older adults higher arterial stiffness could explain increased $\mathrm{CV}$ risk.

\section{EVALUATION OF HABITUAL SALT INTAKE IN SUBJECTS WITH BONE MINERAL METABOLISM DISORDERS}

\author{
Abate V(1), Evangelista M(1), Giaquinto A(1), \\ Cardamone $\mathrm{A}(1)$, Varriale $\mathrm{GA}(1)$, Vollero $\mathrm{M}(1)$, \\ Barone B(2), Rendina D(1), Prezioso D(2), Strazzullo \\ $\mathrm{P}(1)$, Galletti $\mathrm{F}(1)$ \\ 1)Dept. Clinical medicine and surgery AOU Policlinico \\ Federico II Napoli; 2)Dip. Urology AOU Policlinico \\ Federico II Napoli
}

INTRODUCTION: Osteoporosis (OP) and Paget's disease of bone (PDB) are the two most common bone mineral metabolism disorders. Among the therapeutic management of this pathologies, it is commonly recommended a normocalcic and hyposodic diet, even considering the frequent association with cardiovascular diseases. AIM: To evaluate the habitual salt intake of subjects with $\mathrm{OP}$ and PDB.

METHODS: Were evaluated all adult subjects, both sexes, who went to the bone mineral metabolism diseases ambulatory of the Azienda Universitaria Federico II hospital, in the time interval between 1st May 2019 and 1st September 2019 affected by OP and PDB. The habitual salt intake and the consciousness of its use were evaluated using the MINISAL-SIIA questioner.

RESULTS: Were examined 198 subjects with OP or PDB (mean age $64.3 \pm 13.1$ year, BMI: $26.6 \pm 4.5 \mathrm{~kg} / \mathrm{m}^{2}$ ). The average MINISAL-SIIA score is $8.9 \pm 1.6$. The percentage of subjects with MINISAL-SIIA score that identifies high salt consumption (equal or more than 10) was $28.3 \%$. The percentage of subjects who correctly indicates the daily salt intake recommended by World Health Organization is $73.3 \%$.

CONCLUSIONS: Despite hyposodic diet is the pivot of secondary prevention of bone mineral metabolism disorders, our studies demonstrate a reduced adherence to the hyposodic diet, and a reduced knowledge of the correct daily salt intake recommended by World Health Organization in subjects with OP and/or PDB.

\section{EVALUATION OF HABITUAL SALT INTAKE OF IN SUBJECTS WITH RECURRENT NEPHROLITHIASIS}

Evangelista M(1), Abate V(1), Giaquinto A(1), Cardamone A(1), Varriale GA(1), Vollero $M(1)$, Barone B(2), Rendina D(1), Prezioso D(2), Strazzullo $\mathrm{P}(1)$, Galletti F(1)

1) Department of Clinical Medicine and Surgery, $A O U$ Policlinico Federico II Napoli; 2) Department of Urology AOU Policlinico Federico II Napoli, Italy

INTRODUCTION: Nephrolithiasis (NL) is a common multifactorial disease associated with high morbidity if not correctly diagnosed and treated. NL is characterized by an increasing incidence and prevalence in the adult population of industrialized countries, most evident in the females. For the primary and secondary prevention of NL, it is recommended a normocalcic hyposodic diet associated with hydropinic therapy.

AIM: to evaluate the habitual salt intake of subjects with recurrent NL.

METHODS: Were evaluated all adult subjects, both sexes, who went to the extracorporeal lithotripsy (ESWL) ambulatory of the Azienda Universitaria Federico II hospital, in the time interval between 1st May 2019 and 1st September 2019 affected by idiopathic NL. The habitual salt intake and the consciousness of its use were evaluated using the MINISAL-SIIA questioner.

RESULTS: Were examined 69 male subjects treated with ESWL (mean age $50.3 \pm 13.5$ yr, BMI: $27.7 \pm 4.2 \mathrm{~kg} / \mathrm{m}^{2}$ ) and 68 female subjects treated with ESWL (mean age $55.0 \pm 10.8$ yr, BMI: $28.0 \pm 5.9 \mathrm{~kg} / \mathrm{m}^{2}$ ). The average MINISAL-SIIA score is $9.0 \pm 1.2$ in male and $9.0 \pm 1.5$ in female subjects $(\mathrm{p}<0.05)$. The percentage of subjects with MINISAL-SIIA score that identifies high salt consumption (equal or more than 10) is $30.4 \%$ in male and $35.3 \%$ in female subjects $(\mathrm{p}<0.05)$. The percentage of subjects who declare to eat cheese and cured meats 3 or more times a week is significantly higher in males on females $(42 \%$ vs $26 \mathrm{~m} 4 \%, \mathrm{p}=0.03)$. The percentage of subjects who correctly indicates the daily salt intake recommended by World Health Organization is $62.3 \%$ of male and $69.1 \%$ of female subjects.

CONCLUSIONS: Despite hyposodic diet is the pivot of primary and secondary prevention of nephrolithiasis, our studies demonstrate a reduced adherence to the hyposodic diet, and a reduced knowledge of the correct daily salt intake recommended by World Health Organization. 


\section{EFFECT OF CONTINUOUS POSITIVE AIRWAY PRESSURE TREATMENT ON GLYCEMIC AND LIPID PROFILES IN PATIENTS WITH OBSTRUCTIVE SLEEP APNEA: A SYSTEMATIC REVIEW AND META-ANALYSIS}

Filippo Cattazzo ${ }^{1}$, Martino F. Pengo ${ }^{2}$, Alice Giontella ${ }^{1}$, Davide Soranna ${ }^{2}$, Janaka Karalliedde ${ }^{3}$, Luigi Gnudi ${ }^{3}$, Pietro Minuz ${ }^{1}$, Carolina Lombardi ${ }^{2,4}$, Gianfranco Parati $^{2,4}$, Cristiano Fava ${ }^{1}$

\section{Department of Medicine, University of Verona,} Section of General Medicine and Hypertension, Verona, Italy; 2. IRCCS, Istituto Auxologico Italiano; Department of Cardiovascular, Neural and Metabolic Sciences, San Luca Hospital, Milan, Italy; 3. School of Cardiovascular Medicine \& Sciences, Section Vascular Biology and Inflammation, Unit for Metabolic Medicine, King's College London, London, UK; 4 Department of Medicine and Surgery, University of Milano-Bicocca, Milan, Italy

INTRODUCTION: Continuous Positive Airway Pressure (CPAP) is the main therapy for obstructive sleep apnoea (OSA); nevertheless, uncertainty remains about the effectiveness of CPAP not only in controlling OSA-related hypertension, but also in improving the metabolic dysregulation that characterizes OSA patients.

AIM: This meta-analysis of randomized controlled trials (RCTs) aimed to investigate whether CPAP therapy, compared to other control treatments (sham CPAP, oral placebo or standard care), could improve glucose metabolism (fasting plasma glucose [FPG] or glycated haemoglobin or fasting plasma insulin [FPI] or homeostasis model assessment [HOMA] of insulin resistance [IR]) and/ or lipid metabolism (total, HDL, LDL-cholesterol or triglycerides) in OSA patients.

METHODS: Relevant papers were searched in three different databases (MEDLINE, EMBASE and Web of Science) from inception to 31st Dec 2018 through specific search terms and selection criteria.

RESULTS: From 2289 articles, 26 RCTs were included. Regarding glycaemic metabolism, neither FPG nor glycated haemoglobin were reduced by CPAP. However, patients on CPAP therapy showed an improvement in insulin sensitivity, as witnessed by a significant reduction of FPI (Standardized difference in means $[\mathrm{SDM}]=0.205$ [95\% CI 0.07-0.339], $\mathrm{p}=0.003)$ and HOMA IR (SDM = 0.176 [95\% CI 0.056-0.296], $\mathrm{p}=0.004$ ). Sensitivity analysis revealed that the favourable effects of CPAP were maintained in prediabetic/type 2 diabetic patients (considering only FPI) and in those presenting an apnoea-hypopnea index 30 events/h. Regarding lipid metabolism, OSA patients in CPAP intervention groups showed a significant reduction in total cholesterol $(\mathrm{TC} ; \mathrm{SDM}=0.138[95 \% \mathrm{CI}$ $0.034-0.242], p=0.009$ ). In subgroups analysis, greater adherence to CPAP therapy along with severe nocturnal oxygen desaturations at baseline ( $\mathrm{SpO} 2$-nadir $<77 \%$ ) was associated with a significant decrease of TC. Furthermore, dividing the primary studies according to the median TC at baseline $(198 \mathrm{mg} / \mathrm{dL})$, the positive effects of CPAP were maintained only in those that presented a TC above the median. Conversely, CPAP did not modify triglycerides, HDL-, and LDL-cholesterol.

CONCLUSIONS: CPAP treatment significantly improves insulin sensitivity and reduces TC in OSA patients but with a low effect size. The effectiveness of CPAP therapy is higher in patients with greater CPAP usage, and with a greater number of apnoeic events and oxygen desaturations at baseline. Concerning TC, patients presenting higher levels at baseline benefit the most from CPAP.

\section{THE COMPLEX RELATIONSHIP BETWEEN SERUM URIC ACID, ENDOTHELIAL FUNCTION AND SMALL VESSEL REMODELING IN HUMANS}

Stefano Masi 1, 2, 3*, Georgios Georgiopoulos 4, 5*, George Alexopoulos 6, Konstantinos Pateras 6, Javier Rosada 7, Gino Seravalle 8, Carolina De Ciuceis 9, Stefano Taddei 1, Claudio Borghi 10, Guido Grassi 11, Damiano Rizzoni 9, 12*, Agostino Virdis 1* and the Study Groups on the Uric Acid Right for heArt Health (URRAH) $\uparrow$ and Micro- and Macro-circulation of the Italian Society of Hypertension (SIIA)

1. Department of Clinical and Experimental Medicine, University of Pisa, Pisa, Italy; 2. National Centre for Cardiovascular Prevention and Outcomes, Institute of Cardiovascular, Science, University College London, London, UK; 3. Department of Twin Research \& Genetic Epidemiology, King's College London, London, UK; 4. School of Biomedical Engineering and Imaging Sciences, King's College London, London, UK; 5. Department of Clinical Therapeutics, National and Kapodistrian University of Athens, Greece; 6. Department of Biostatistics and Research Support, Julius Center for Health Sciences and Primary Care, University Medical Center Utrecht, Utrecht, The Netherlands; 7. Fourth Unit 
of Internal Medicine, University Hospital of Pisa, Pisa, Italy; 8. Cardiology Unit, Fondazione Istituto Auxologico Italiano, Ospedale S. Luca, IRCCS Istituto Auxologico Italiano, Milan, Italy; 9. Department of Clinical and Experimental Sciences, University of Brescia, Italy; 10. Department of Medical and Surgical Science, Alma Mater Studiorum University of Bologna, Italy; 11. Clinica Medica, Deptartment of Health Science, University Milano-Bicocca, Italy; 12. Division of Medicine, Spedali Civili di Brescia, Montichiari Hospital, Brescia, Italy. $*$ These authors equally contributed to the manuscript; $\dagger$ Name and affiliations of the study group members are reported in the acknowledgements

INTRODUCTION: The relationship between serum uric acid (SUA) and microvascular remodeling in humans remains largely unexplored.

AIM: We assessed whether SUA provides additional information on the severity of microvascular remodeling than that obtained from the European Heart Score (HS), the patterns of microvascular remodeling associated with changes in SUA levels and the mediation by endothelial function and NO availability on this relationship.

METHODS: A total of 162 patients included in the microvascular dataset of the Italian Society of Hypertension with available information on SUA, media-to-lumen $(\mathrm{M} / \mathrm{L})$ ratio, media cross-sectional area (MCSA), endothelial function, $\mathrm{NO}$ availability and $\mathrm{HS}$ were included in the analysis. The top tertile of $\mathrm{M} / \mathrm{L}$ ratio and MCSA were used to define severe microvascular remodeling.

RESULTS: A U-shaped association was observed between SUA and both M/L ratio and MCSA. Adjustment for HS did not affect these associations. SUA was able to reclassify a significant amount of subjects without and with severe $\mathrm{M} / \mathrm{L}$ ratio and MCSA remodeling over the HS alone. The microvascular remodeling associated with SUA levels presented a predominant hypertrophic pattern. SUA was inversely associated with endothelialfunction and $\mathrm{NO}$ availability. Structural equation modeling analysis controlling for the HS suggested that the association of SUA with $\mathrm{M} / \mathrm{L}$ ratio and MCSA was mediated through changes in endothelial function and $\mathrm{NO}$ availability.

CONCLUSIONS: The addition of SUA to the HS improves the identification of subjects with greater microvascular remodeling. The relationship between SUA and microvascular remodeling is mediated by endothelial function and NO availability.
APPLICATION OF THE SAMPSON FORMULA TO ESTIMATE LDL-C IN CHILDREN: COMPARISON WITH DIRECT LDL MEASUREMENT AND FRIEDEWALD FORMULA IN THE BLIP STUDY

Arrigo F.G. Cicero ${ }^{1}$, Federica Fogacci ${ }^{1}$, Daniela Patrono $^{2}$, Rita Mancini ${ }^{2}$, Arianna Rossolini ${ }^{1}$, Claudio Borghi ${ }^{1}$, Sergio D' Addato ${ }^{1}$ on behalf of the BLIP Study group*

${ }^{1}$ Medical and Surgical Sciences Dept., Alma Mater Studiorum University of Bologna, Bologna, Italy; ${ }^{2}$ Unified Metropolitan Laboratory (LUM), AOU S. OrsolaMalpighi, Bologna, Italy

INTRODUCTION: In epidemiological trials and in clinical practice, it is relevant to have cheap and reliable methods to measure the main lipid cardiovascular risk factor, i.e. low-density lipoprotein cholesterol (LDL-C) plasma level.

AIM: In this context, we aimed to compare the reliability of the Friedewald's and Sampson's equations with the LDL-value measured by a validated direct method in a large cohort of children.

METHODS: We considered the lipid values of 145 infants, 278 preschoolers, 810 scholar children, and 1372 adolescents (Total N. 2605, 1291 males, 1314 females), with mean $\mathrm{TC}=169.8 \pm 39.7 \mathrm{mg} / \mathrm{dL}$, HDL$\mathrm{C}=50.8 \pm 12.7 \mathrm{mg} / \mathrm{dL}$, non HDL-C $=118.9 \pm 35.9 \mathrm{mg} /$ $\mathrm{dL}, \quad \mathrm{TG}=90.3 \pm 77.9 \mathrm{mg} / \mathrm{dL}, \quad$ LDL-Cd $=106.2 \pm 29.9$ $\mathrm{mg} / \mathrm{dL}, \quad \mathrm{LDL}-\mathrm{Cf}=100.9 \pm 33.8 \mathrm{mg} / \mathrm{dL}$, and LDL-Cs $=$ $102.2 \pm 33.4 \mathrm{mg} / \mathrm{dL}$.

RESULTS: Comparing the distance to the LDL-Cd, Friedewald's formula mildly but significantly underestimated in infants $(3.4 \pm 5.3 \mathrm{mg} / \mathrm{dL})$, preschoolers $(1.5 \pm 7.1 \mathrm{mg} / \mathrm{dL})$. Children $(1.2 \pm 2.2 \mathrm{mg} / \mathrm{dL})$ and adolescents $(1.1 \pm 5.9 \mathrm{mg} / \mathrm{dL})$ compared to Sampson's formula (all comparisons, $\mathrm{p}<0.001$ ) (see figure136_20200617133601).

CONCLUSIONS: Our analysis, being carried out on a large population sample, shows that Sampson's formula is more reliable than Friedewald's one at each considered age class and even for extreme TG values. 


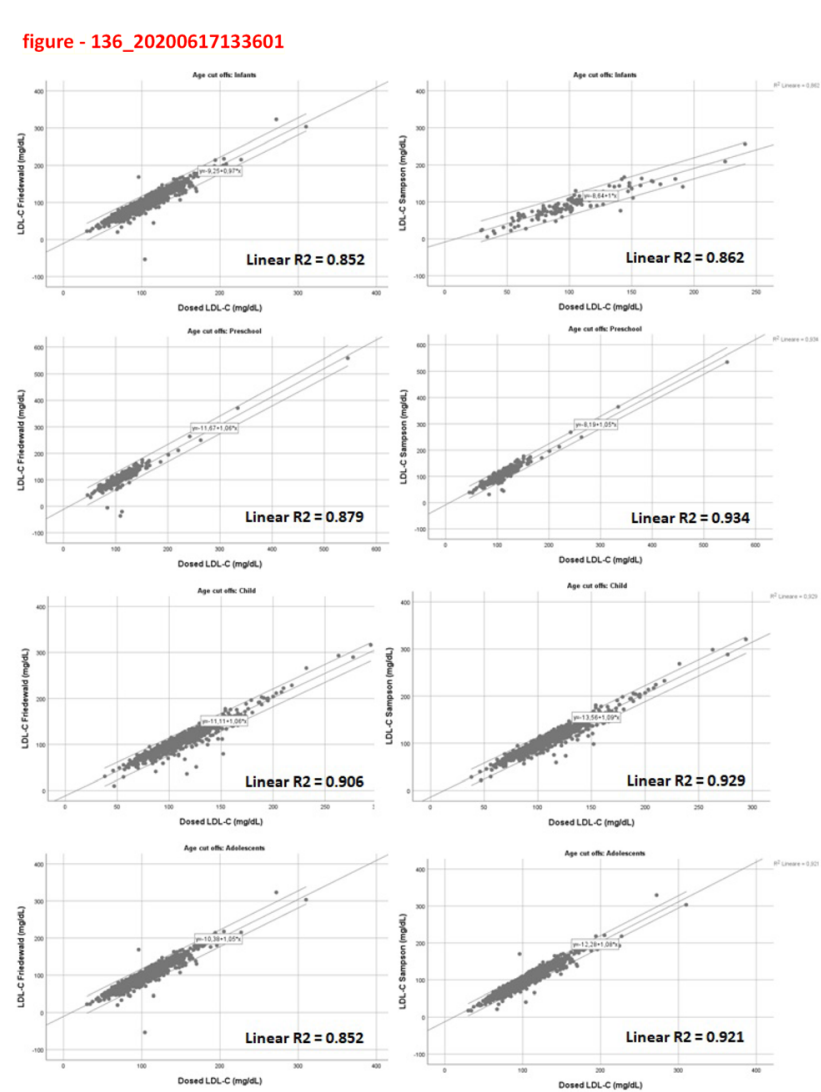

\section{THE IMPORTANCE OF INCLUDING URIC ACID IN THE DEFINITION OF METABOLIC SYNDROME WHEN ASSESSING THE MORTALITY RISK}

Nicola Riccardo Pugliese ${ }^{1 *}$, Stefano Masi ${ }^{1 *}$, Edoardo Casiglia $^{2}$, Valerie Tikhonoff ${ }^{2}$, Arrigo F.G. Cicero ${ }^{3}$, Andrea Ungar ${ }^{4}$, Giulia Rivasi ${ }^{4}$, Massimo Salvetti ${ }^{5}$, Carlo M. Barbagallo ${ }^{6}$, Michele Bombelli ${ }^{7}$, Raffaella Dell'Oro $^{7}$, Berardino Bruno ${ }^{8}$, Luciano Lippa ${ }^{9}$, Lanfranco D'Elia ${ }^{10}$ Agostino Virdis ${ }^{1}$ and Claudio Borghi $^{3}$, from the Working Group on Uric Acid and Cardiovascular Risk of the Italian Society of Hypertension

${ }^{I}$ Department of Clinical and Experimental Medicine, University of Pisa, Pisa, Italy; ${ }^{2}$ Department of Medicine, University of Padua, Padua, Italy; ${ }^{3}$ Department of Medical and Surgical Science, Alma Mater Studiorum University of Bologna, Italy; ${ }^{4}$ Department of Geriatric and Intensive Care Medicine, Careggi Hospital and University of Florence, Italy; ${ }^{5}$ Department of Clinical and Experimental Sciences, University of Brescia, Italy; ${ }^{6}$ Biomedical Department of Internal Medicine and Specialistics, University of Palermo, Italy; ${ }^{7}$ Clinica Medica, Department of Medicine and Surgery, University of Milano-Bicocca, Monza, Italy; ${ }^{8}$ Department of Life, Health and Environmental Sciences, University of L'Aquila, Italy; ${ }^{9}$ Italian Society of General Medicine, Avezzano, L'Aquila, Italy; ${ }^{10}$ Department of Clinical Medicine and Surgery, Federico II University of Naples Medical School, Italy

INTRODUCTION: Serum uric acid (SUA) can stratify patients with different cardiovascular risk and has been depicted as a contributory causal factor in metabolic syndrome (MS), which in turn portends an unfavourable prognosis.

AIM: We assessed the independent prognostic role of SUA in patients with MS.

METHODS: We used data from the multicentre, retrospective, observational URRAH (Uric Acid Right for Heart Health), which recruited subjects from hypertension clinics. The outcome we considered was cardiovascular mortality (CVM), defined as death due to fatal myocardial infarction, stroke, sudden cardiac death, or heart failure.

RESULTS: A total of 9589 subjects (median age 58.5 years old, $45 \%$ males) were included in the analysis, and $5100(53 \%)$ patients had a final diagnosis of MS. After a median follow-up of 142 months, we observed 558 events. Using a previously validated cardiovascular SUA cut-off to predict CVM $(>5.1 \mathrm{mg} / \mathrm{dL}$ in women and $5.6 \mathrm{mg} / \mathrm{dL}$ in men), elevated SUA levels were significantly associated to a worse outcome in patients with and without MS (all $\mathrm{p}<0.0001$ ) and provided a significant net reclassification improvement of $5.1 \%$ over the diagnosis of MS for CVM $(p=0.004)$. Multivariate Cox regression analyses identified an independent association between SUA and CVM (Hazard Ratio: 1.79 [95\% CI 1.15-2.79]; p < 0.001) after the adjustment for MS, its single components and other common $\mathrm{CV}$ risk factors.

CONCLUSIONS: Increasing SUA levels are associated with a higher risk of CVM irrespective of the presence of the MS. A cardiovascular SUA threshold may improve cardiovascular risk stratification in patients with MS. 


\section{GENDER RELATED DIFFERENCES IN TWO TYPES OF WEIGHT LOSS INTERVENTION}

\author{
M. D'Abbondanza ${ }^{1}$, S. Ministrini ${ }^{1}$, G. Mondovecchio ${ }^{1}$, \\ E. E. Martorelli ${ }^{1}$, M. A. Ricci ${ }^{1}$, S. De Vuono ${ }^{1}$, E. Nulli \\ Migliola $^{1}$, A. R. Roscini ${ }^{1}$, D. Siepi ${ }^{1}$, M. T. Paganelli ${ }^{2}$, \\ G. Lupattelli ${ }^{1}$ \\ ${ }^{1}$ Internal Medicine, Department of Medicine, "Santa \\ Maria della Misericordia” Hospital, University \\ of Perugia-Italy, ${ }^{2}$ Unit of General Surgery, "Santa Maria \\ della Misericordia" Hospital, Perugia -Italy
}

INTRODUCTION: historically, women have been underrepresented in clinical trials: particularly, only a few studies described gender effect on surgical and non-surgical interventions, with discordant results.

AIM: to investigate whether and how gender influences weight loss (WL) in two different populations, after sleeve gastrectomy and Ketogenic diet.

METHODS: 125 patients with morbid obesity (BMI 40 $\mathrm{kg} / \mathrm{m}^{2}$ or $35 \mathrm{~kg} / \mathrm{m}^{2}+$ comorbidity) eligible to sleeve gastrectomy (group1) and a group of 40 patients following a ketogenic diet (group2). We evaluated anthropometric parameters, peripheral $\mathrm{BP}$, biochemical and serum analysis at the enrollment and at twelve months after interventions. RESULTS: in the whole population there was a female prevalence (70\% in group1 and $60 \%$ in group2). Males presented increased adiposity (measured as VFA and fat mass) in both groups and a worse glyco-metabolic profile in group1. Only after ketogenic diet we observed a significant difference between sexes in total body WL (7.4\% in males vs $5.9 \%$ in females, $\mathrm{p}<0.05$ ) and excess of body WL $(15.7 \%$ in males vs $12.1 \%$ in females, $\mathrm{p}<0.05)$ in favour of male population. However, only in group1, we observed a larger and significant improvement in adiposity (VFA, fat mass) and glyco-metabolic (HOMA-IR, HbA1c) parameters in males rather than in females.

CONCLUSIONS: male subjects, beyond a higher weight and BMI, had a worse glyco-metabolic pattern. No significant difference between sexes was found in WL in group 1 patients; on the contrary, male patients seemed to have a major benefit in WL from ketogenic diet in comparison to female population. In both groups, male sex was an independent predictor of EBWL. Gender differences in WL intervention could be determined by several factors: hormonal profiles, different body composition or higher initial weight in males. Further studies are necessary to investigate the determinants of these differences.

\section{FUNCTIONAL CAPACITY AND COMORBIDITIES IN PATIENTS WITH MODERATE AND SEVERE OBESITY}

Francesca Battista ${ }^{1}$, Neunhäuserer Daniel ${ }^{1}$, Quinto Giulia $^{1}$, Frigo Anna Chiara ${ }^{2}$, Bianchi Elia ${ }^{1}$, Gobbo Stefano $^{1}$, Ortolan Sara ${ }^{1}$, Gasperetti Andrea ${ }^{1}$, Ermolao Andrea $^{1}$

1-Department of Medicine, Sports and Exercise Medicine Division, University of Padova; Regional Center for Exercise Prescription in Chronic Diseases, Veneto Region, Italy; 2-Department of Cardiac, Thoracic and Vascular Sciences, Biostatistics, Epidemiology and Public Health Unit, University of Padova, Padova, Italy

INTRODUCTION: Cardiorespiratory fitness is a strong predictor of morbidity and mortality also in people with obesity. However, no reference values of aerobic capacity are currently available for subjects with severe obesity and comorbidities.

AIM: This study describe the distribution of aerobic capacity in a group of patients with severe obesity and its relationship with comorbidities.

METHODS: observational study on 542 patients $(69 \%$ Females) with $\mathrm{BMI} \geq 30 \mathrm{~kg} / \mathrm{m}^{2}$ that consecutively performed a Cardiopulmonary Exercise Test (CPET) by using incremental ramp treadmill test (modified Bruce protocol) or bicycle ergometer test $(+15 \mathrm{~W} /$ minute). Anthropometric and clinical data were recorded simultaneously.

RESULTS: The median (IQR) age is 47.0(62) years, mean BMI is $41.7 \pm 6.7 \mathrm{~kg} / \mathrm{m}^{2}$. Normal values curves have been developed for relative VO2peak (VO2peak/Kg) that shows mean value of $20.9 \pm 4.8 \mathrm{ml} / \mathrm{min} / \mathrm{Kg}$ (median $/ \mathrm{IQR}=20.3 /$ $37.6 \mathrm{ml} / \mathrm{min} / \mathrm{Kg}$ ). Upper limit of the lower quartile of VO2peak $/ \mathrm{kg}$ is $17.87 \mathrm{ml} / \mathrm{min} / \mathrm{Kg}$. Mean absolute VO2peak is $2.410 \pm 6.7 \mathrm{~L} / \mathrm{min}$. Analysis of covariance (ANCOVA) displays that VO2peak/kg inversely correlates with age ( $\mathrm{p}$ $0.0001)$ and BMI (p 0.0001) and also a significant effect of the interaction term age*BMI (p 0.0001). ANCOVA of absolute VO2peak shows direct correlation with BMI ( $\mathrm{p}$ 0.0001 ), inverse correlation with age ( $p$ 0.0001) and significant effect of the interaction term age*gender $(\mathrm{p}$ 0.0006). Both relative and absolute VO2peak are lower in females than in males (p 0.0001). A multivariate logistic regression model points out that the odds of belonging to the lower quartile of $\mathrm{VO} 2$ peak $/ \mathrm{kg}$ is independently determined by age (age 47-54 years OR 2.549 IC 95\% $1.205-5.392 \mathrm{p}<0.0001)$ and BMI $\left(\mathrm{BMI}>45 \mathrm{~kg} / \mathrm{m}^{2}\right.$ OR 5.864 IC $95 \% 2.920-11.778 \mathrm{p}<0.0001)$, but not by number of comorbidities. 
CONCLUSIONS: VO2peak/Kg decreases with increasing age and BMI both in males and females, but at highest BMI $\left(>45 \mathrm{~kg} / \mathrm{m}^{2}\right)$ the age effect is lower. Age and very high BMI, but not comorbidities, are independent determinants of low VO2peak/kg

\section{DEVELOPMENT OF SYSTOLIC DYSFUNCTION IN HYPERTENSION}

Dario Tino Bertolone, Eva Gerdts, Richard B.

Devereux, Cristina De Colle, Nicola De Luca, Giovanni de Simone, Costantino Mancusi

\section{Hypertension Research Center, Federico II University Hospital, Naples, ITALY}

INTRODUCTION: While it is commonly thought that left ventricular (LV) systolic function may insidiously deteriorate in hypertensive patients, few prospective data are available to support this notion.

METHODS: We evaluated 680 hypertensive patients (66 \pm 7 years; $45 \%$ women) with ECG-LV hypertrophy enrolled in the LIFE echo-substudy. Only patients free of prevalent cardiovascular disease and with baseline ejection fraction $(\mathrm{EF}) \geq 55 \%$ were included. Echocardiographic exams were performed annually for 5 years during antihypertensive treatment. Development of reduced systolic function was defined as incident $\mathrm{EF}<50 \%$.

RESULTS: During a mean follow up of $4.8 \pm 1$ years, 37 patients developed reduced EF without an intercurrent myocardial infarction (5.4\%). Patients who developed reduced EF were more often men $(\mathrm{p}<0.05)$. In analysis of covariance, patients who developed reduced EF had greater baseline LV diameter and LV mass, lower mean EF (all $\mathrm{p}<0.05)$, and similar diastolic function indices. At last available exam before $\mathrm{EF}$ reduction, independently of covariates, patients with reduced $\mathrm{EF}$ showed a significant increase in LA size, LV diameter, end-systolic stress and mitral E/A ratio, as compared to those who did not develop reduced $\mathrm{EF}$ (all $\mathrm{p}<0.05$ ). In time-varying Cox regression analysis, also controlling for baseline EF, predictors of developing reduced EF were higher in-treatment LV diameter $(\mathrm{HR}=5.19$ per $\mathrm{cm} ; 95 \%$ CI 2.58-10.41) and higher in-treatment mitral $\mathrm{E} / \mathrm{A}$ ratio $(\mathrm{HR}=2.37 ; 95 \% \mathrm{CI}$ $1.58-3.56$; both $\mathrm{p}<0.0001)$.
CONCLUSIONS: In treated hypertensive patients, incident reduced EF is associated with the development of dilated LV chamber and signs of increased LV filling pressure. Higher in-treatment LV diameter and mitral E/A ratio are the strongest echocardiographic predictors of reduced $\mathrm{EF}$

\section{AWARENESS OF MODIFIABLE CARDIOVASCULAR RISKS FACTORS}

Cristina De Colle, Francesco Rozza, Raffaele Izzo, Nicola De Luca, Dario Tino Bertolone, Ilaria Fucile, Giovanni de Simone, Costantino Mancusi

Hypertension Research Center, Federico II University Hospital, Naples, ITALY

INTRODUCTION: Cardiovascular risks factors are specific conditions that are associated with increased risk of cardiovascular disease. Awareness of modifiable cardiovascular risks factors is of paramount importance in secondary $\mathrm{CV}$ prevention.

AIM: to assess awareness of modifiable cardiovascular risks factors among patients with recent acute myocardial infarction (AMI).

METHODS: We interviewed fifty patients (38 men; 12 women) with AMI (50\% STEMI; 50\% NSTEMI), admitted to Cardiology Rehabilitation Unit of the Federico II university hospital, Naples, from June 2019 to December 2019. We submitted them a questionnaire concerning awareness of life style cardiovascular risks factors.

RESULTS: In our study's population the whole sample (100\% of patients) identified like CV risk factors hypertension, hypercholesterolemia and obesity; $20 \%$ of patients do not recognize conditions like diabetes mellitus and smoke as CV risk factors; moreover, most patients did not recognize daily physical activity and in Mediterranean diet protective factors for cardiovascular disease (respectively $37 \%$ and $44 \%$ of patients). Patients with AMI-NSTEMI had more awareness of modified cardiovascular risks factors compared to patients with AMI-STEMI.

CONCLUSIONS: Among patients admitted to Cardiac Rehabilitation Unit for acute AMI awareness of modifiable $\mathrm{CV}$ risk factors is suboptimal. In particular physical activities and Mediterranean diet are not recognize as important life style modification for secondary $\mathrm{CV}$ 
prevention. Therefore the important aim for patient care purposes is information and education about life style advices.

\section{Atherosclerosis and Inflammation}

\section{GINGIVAL BLEEDING IS ASSOCIATED WITH HIGH BLOOD PRESSURE}

Rita DEL PINTO ${ }^{\text {a }}$, Davide PIETROPAOLI ${ }^{\text {a }}$ *, Annalisa MONACO ${ }^{\text {a }}$, Francesco D’AIUTO ${ }^{\text {b }}$, Eva MUÑOZ AGUILERA ${ }^{\mathrm{b}}$, Eleonora ORTU ${ }^{\mathrm{a}}$, Mario

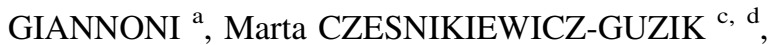
Tomasz J. GUZIK ${ }^{\mathrm{c}, \mathrm{d}}$, Claudio FERRI ${ }^{\mathrm{a}}$

${ }^{a}$ University of L'Aquila, L'Aquila (Italy); ${ }^{b}$ University College London, London (UK); ${ }^{c}$ University of Glasgow, Glasgow (UK); ${ }^{d}$ Jagiellonian University, Collegium Medicum, Krakow (PL)

INTRODUCTION: Cardiovascular disease (CVD) and related risk factors, including hypertension, are characterized by systemic inflammation. Periodontitis, a chronic inflammatory disease of the supporting tissues of the tooth, can impact the systemic inflammatory burden and has recently been associated with hypertension. However, it is not clear whether the risk of hypertension can vary throughout the natural history of periodontal diseases, that is, if it is modified by gingival bleeding, an easily investigable indicator of local pathology.

METHODS: Survey-based propensity score matching (PSM) incorporating major confounders shared between hypertension and periodontal diseases (age, gender, ethnicity, poverty, BMI, hypertension diagnosis and treatment, smoking habit, diabetic status, and aspirin use) was applied to cross-sectional NHANES III data from adults $\geq 30$ years who underwent BP measurement and periodontal examination, identifying two matched groups with and without gingival bleeding. The association of bleeding gums with systolic blood pressure (BP, $\mathrm{mmHg}$ ) and high/ uncontrolled BP according to the European Society of Hypertension (ESH) was then assessed with generalized additive models incorporating inflammatory markers (CRP, white blood cells, ferritin). Stratification by periodontal health status (healthy; gingivitis; stable periodontitis; unstable periodontitis) was performed.

RESULTS: Gingival bleeding (gingivitis; unstable periodontitis) was independently associated with $+2.6 \mathrm{mmHg}$ ( $p<0.001)$ systolic BP compared with no bleeding (healthy periodontium; stable periodontitis), and with greater odds (OR 1.42; 95\% CI 1.19-1.68; p < 0.001) of high/uncontrolled BP. Participants with unstable periodontitis had higher systolic BP than those with stable periodontitis $(+2.1 \mathrm{mmHg} ; \quad \mathrm{p}<0.001) \quad$ or gingivitis $(+5.3 \mathrm{mmHg} ; \mathrm{p}<0.001)$. Unstable periodontitis and gingivitis, but not stable periodontitis, were associated with increased risk of high/uncontrolled BP (OR 1.65, 95\% CI 2.14-1.82; OR $1.49,95 \%$ CI 1.22-1.82, respectively).

CONCLUSIONS: Gingival bleeding contributes to shaping the relationship between periodontal diseases and hypertension. Investigating gingival bleeding status could be of importance in hypertensive patients, especially if poorly controlled by therapy.

\section{IN TYPE 1 DIABETES MELLITUS, CAROTID INTIMA-MEDIA THICKNESS SHOWS DIFFERENCES BETWEEN GENDERS EARLY AFTER PUBERTY}

Bortolotti Stefano $^{1}$, Tagetti Angela ${ }^{1}$, Giontella Alice ${ }^{1}$, Branz Lorella ${ }^{1}$, Marcon Denise ${ }^{1}$, Minuz Pietro ${ }^{1}$, Antoniazzi Franco ${ }^{2}$, Maffeis Claudio ${ }^{2}$, Fava Cristiano ${ }^{1}$

${ }^{1}$ Department of Medicine, University of Verona, Italy; ${ }^{2}$ Department of Surgery, Dentistry, Paediatrics and Gynaecology, University of Verona, Italy

INTRODUCTION: Type 1 diabetes mellitus (T1DM) is characterized by the early development of macrovascular damages in both genders but could manifest earlier in males. Traditional risk factors, along with T1DM per se, are associated with this process in T1DM. If specific differences in early vascular damage are viewable even during childhood in T1DM is unknown.

METHODS: Arterial structural changes (carotid intimamedia thickness [cIMT]) and vascular elasticity (carotid coefficient of distensibility [cDC] and Pulse Wave Velocity [PWV]) were measured in 180 children and adolescents with T1DM by carotid ultrasonography and the SphygmoCor XCel device. Hemodynamic and metabolic risk factors (peripheral and central systolic/diastolic blood pressure [pSBP/pDBP; cSBP], body mass index (BMI), glycated haemoglobin, triglycerides, total cholesterol, nonHDL/HDL-cholesterol ratio were also assessed. The sample was divided according to Tunner's stages (pre-pubertal-pubertal vs. post-pubertal) and gender: seventy-four subjects were classified as pre-pubertal-pubertal (44 male, mean age \pm SD $12 \pm 2$ years: 30 females, $12 \pm 1.5$ years) and 106 as post-pubertal (43 males, $16 \pm 1$ years; 63 females; age: $15.5 \pm 1.5$ years). Sex-specific linear regression models, adjusted for the pubertal state, BMI, duration of diabetes, glycated haemoglobin, nonHDL/ HDL-cholesterol ratio, were constructed to assess the 
relation between risk factors and the markers of early vascular damage.

RESULTS No differences in cIMT, cDC, PWV and the respective z-score were found between males and females in the pre-pubertal-prepubertal sub-group. In the post-pubertal group, males had higher cIMT $(0.05 \pm 0.01 \mathrm{~mm}$; $p$ value: 0.001$)$ and $Z$-score cIMT $(0.62 \pm 0.02 ; p$ value: 0.04 ) but no differences in cDC and PWV were found. In linear regression models, the only risk factors independently associated with z-cIMT, were the nonHDL/HDLcholesterol ratio ( $\beta \pm$ SEM: $0.267 \pm 0.30$; $p$ value: 0.03 ) in males and z-cSBP in females ( $\beta \pm$ SEM: $0.286 \pm 0.13$; $\mathrm{p}$ value: 0.01$)$. $\mathrm{z}$-PWV and $\mathrm{z}$-cDC showed associations with different types of BP values both in males and females.

CONCLUSIONS: Gender-specific differences in cIMT are present early after puberty in adolescents with T1DM but seem associated with different risk factors in males and females. Different preventive strategies could be beneficial in males and females with T1DM, starting during adolescence.

\section{PERIPHERAL HYPERTENSIVE STIMULI ACTIVATE SFO AND PVN NEURONS BY STIMULATING THE PRIMING OF ADAPTIVE IMMUNITY IN THE SPLEEN THROUGH INCREASED ACTIVITY OF THE SPLENIC SYMPATHETIC NERVE}

\author{
${ }^{1}$ Perrotta M, ${ }^{2}$ Pallante F, ${ }^{2}$ Carnevale L, ${ }^{2}$ Iodice D, \\ ${ }^{2}$ Fardella V, ${ }^{1,2}$ Lembo G, ${ }^{1,2}$ Carnevale D \\ ${ }^{1}$ Department of Molecular Medicine, Sapienza University \\ of Rome, Italy; ${ }^{2}$ Department of Angiocardioneurology \\ and Translational Medicine, IRCCS Neuromed, Pozzilli, \\ Italy
}

INTRODUCTION: Angiotensin II (AngII) is an important modulator of the sympathetic nervous system, acting through its neuronal AT1 (AT1R) receptors in the main cardiovascular control centers. The subfornical organ (SFO), located in a ventricular region where the blood brain barrier is permeable to circulating peptides as AngII, and the paraventricular nucleus of the hypothalamus (PVN), to which SFO is closely connected, are densely enriched of AT1R. In the past, we have described a vagussplenic nerve pathway responsible for priming $\mathrm{T}$ cells in hypertension, but brain areas have not yet been well identified.

METHODS: To study the role of SFO we injected, through stereotaxis apparatus, a recombinant adenovirus, encoding a Cre-recombinase and a GFP (AdCreGFP), or
GFP alone as control in SFO of AT1 ${ }^{\text {flox }}$ mice (mice with loxP sites upstream and downstream of exon 3 of AT gene type 1a). To study the role of PVN we crossed AT1 a $\mathrm{a}^{\text {flox }}$ mice with Sim1Cre mice, since the Sim1 neurons of PVN express AT1aR and Cre-recombinase on the Sim1 promoter.

RESULTS: After evaluating the gene deletion by PCR on SFO and PVN samples obtained by laser microdissection, we treated mice of the two genotypes chronically with AngII and vehicle. Boh AT1aR ${ }^{\text {flox }}$; AdGFPCre mice and AT1aR ${ }^{\text {flox }}$; Sim1Cre + mice showed a significant reduction of blood pressure levels, as compared to WT control mice after AngII. Interestingly, SSNA in both KO mice for AT1aR was significantly reduced when compared to WT mice as well as the expression of the placental growth factor (PIGF) in the splenic marginal zone and the activation of $\mathrm{T}$ cells from the spleen. To investigate renal organ damage, we observed protection from infiltrating CD4/ CD8 cells in both KO mice for AT1aR after 28 days of AngII.

CONCLUSIONS: These data identify a nervous control axis of immunity in hypertension to block splenic sympathetic activation and adaptive immunity.

\section{CELIAC VAGUS NERVE STIMULATION RECAPITULATES THE ACTIVATION OF SPLENIC NORADRENERGIC PATHWAY INDUCED BY ANGIOTENSIN-II, DRIVING CD8 EFFECTOR CELLS EGRESSION}

Carnevale L, Pallante F, Perrotta M, Iodice D, Perrotta S, Fardella S, Mastroiacovo F, Carnevale D, Lembo G

IRCC Neuromed, Pozzilli (IS), Italy

INTRODUCTION: We previously described how Angiotensin-II (AngII) recruits T-cells through the spleen, homing them to the cardiovascular system and inducing elevated blood pressure (BP). AngII action is mediated by the central nervous system to enhance the sympathetic tone, in this work we aim at characterize the connection between spleen and brain analysing the activity of cholinergic inflammatory pathway (CIP).

METHODS: we recorded splenic and celiac vagus nerve activity, showing how mice infuse with AngII by subcutaneous osmotic pumps show an incremented celiac vagus nerve activity, evidenced by a nervous burst analysis tool. After this, we set up a technique to stimulate the celiac vagus nerve while recording the splenic nerve activity.

RESULTS: Mice subjected to vagus nerve stimulation present an incremented splenic nerve activity, with a consequent splenic noradrenergic pathway recruitment shown 
by an increased noradrenaline and tyroxine hydroxylase expression in the spleen with a subsequent T-cells egression shown with a reduced $\mathrm{CD} 3+$ positive staining in immunofluorescence analysis. Flow cytometry analysis shown a specific reduction in CD8 + effector cells.

CONCLUSIONS: Our experiments shown also that, while not involved in nervous signal transduction to the spleen, the Placental Growth Factor (PIGF) plays a key role in the transduction of the neural signal to an immune response.

\section{EFFECTS OF PCSK-9 INHIBITORS ON CAROTID ATHEROSCLEROSIS}

Elena De Angelis, Rosanna Matturro, Pompea

Bottiglieri, Antonio Pipolo, Claretta Del Prete, Alessandra Milone, Carmine Vecchione, Gennaro Galasso, Michele Ciccarelli

\section{Department of Medicine and Surgery, Division of Cardiology- University of Salerno}

INTRODUCTION: Proprotein convertase subtilisin/ Kexin type 9 (PCSK9) inhibitors reduce LDL-C in statinezetimibe treated patients and improve outcomes of myocardial infarction and stroke. The GLAGOV trial demonstrated that Evolocumab in patients treated with statin therapy had a favourable effect on the progression of coronary atherosclerosis as measured by IVUS. However, the effects of PCSK9 inhibitors on carotid plaques have not yet been evaluated.

METHODS: We enrolled 46 patients aged more than 18 years, with CIMT $>1.1 \mathrm{~mm}$, affected by Heterozygous Familial Hypercholesterolemia (HeFH) with LDL-cholesterol values $>70 \mathrm{mg} / \mathrm{dL}$ in secondary prevention or $>130$ $\mathrm{mg} / \mathrm{dL}$ in primary prevention or affected by non-Familiar Dyslipidaemia or Mixed Dyslipidaemia in secondary prevention with LDL-cholesterol levels $>100 \mathrm{mg} / \mathrm{dL}$. All patients were on maximum tolerated statin dosage and ezetimibe and treated by evolocumab or alirocumab. Supra-aortic trunks echo-colour-Doppler was performed before starting the therapy (time 0 ), after 6 months and then after 12 months of therapy. For each carotid CIMT, PSV (Peak Systolic Velocity), EDV (End Diastolic Velocity) and ICA/CCA PSV ratio were measured.

RESULTS: We observed after 6 months a reduction of right mean CIMT of -0.09 ( $p=0.000$ vs. baseline) and of -0.14 of left CIMT ( $p=0.008$ vs. baseline); the reduction was for right CIMT after 12 months of -0.16 ( $p=0.006$ vs 6 months) and for left CIMT of -0.15 ( $p=0.005$ vs 6 months). Between 0 time and 12 months of treatment we observed a reduction of $-0.16(p=0.05)$ for right CIMT and of $-0.18(\mathrm{p}=0.004)$ for left CIMT. LDL-
$\mathrm{C}$ levels were reduced from $134 \pm 35.73 \mathrm{mg} / \mathrm{dL}$ at baseline to $65.61 \pm 41.43 \mathrm{mg} / \mathrm{dL}$ after 6 months $(\mathrm{p}=0.000)$ and to $51.50 \pm 18.67 \mathrm{mg} / \mathrm{dL}$ after 12 months $(\mathrm{p}=0.000)$.Moreover, we observed a progressive "remodeling" of the plaques, modifying from a soft/dense composition to a predominantly fibro-calcific composition ( $\mathrm{p}=0.000$ for 0 vs. 12 months).

CONCLUSIONS: This is the first study showing that the inhibitors of PCSK9, can decrease or even reverse the progression of carotid atherosclerosis, reducing the intimamedia thickness, the lipid content of atheromatous plaques leading to a reduction of clinical adverse events.

Heart

THE ROLE OF SACUBITRIL-VALSARTAN IN THE
TREATMENT OF CHRONIC HEART FAILURE
WITH REDUCED EJECTION FRACTION IN
HYPERTENSIVE PATIENTS WITH
COMORBIDITIES: FROM CLINICAL TRIALS TO
REAL-WORLD SETTINGS

Gioia Torin ${ }^{1,2}$, Laura Schiavon ${ }^{1,2}$, Cuppini Stefano ${ }^{2}$, Pietro Minuz ${ }^{3}$, Alberto Mazza ${ }^{1,2}$

1.ESH Excellence Hypertension Centre, Internal Medicine Unit, S. Maria della Misericordia General Hospital, AULSS 5 Polesana, Rovigo, Italy.; 2.Unit of Internal Medicine, S. Maria della Misericordia Hospital, AULSS 5 Polesana, Rovigo, Italy.; 3. Unit of Internal Medicine C, Department of Medicine, University of Verona, Verona, Italy

INTRODUCTION: the effectiveness and safety of Sacubitril-Valsartan has demonstrated to be effective in heart failure with reduced ejection fraction (HFrEF)

AIM: to evaluate the effectiveness and safety of SacubitrilValsartan (SV) in elderly hypertensive patients with comorbidities and HFrEF.

METHODS: In this prospective open-label study 54 subjects (mean age $78.6 \pm 8.2$ years, $75.0 \%$ male), with $\mathrm{HFrEF}$ $(29.8 \pm 4.3 \%)$ and with a New York Heart Association (NYHA) class II-III symptoms were assigned to receive SV. These patients were gender and age-matched with a control arm of patients with HFrEF receiving the optimal standard therapy for HF before the introduction in the market of SV. The clinic blood pressure (BP), N-terminal pro-B-type natriuretic peptide (NT-proBNP), estimated glomerular filtration rate (eGFR), blood glucose and glycated haemoglobin (HbA1c), uric acid (UA), left ventricular ejection fraction (LVEF) and NYHA class were evaluated at a mean follow-up of 12 months. 
RESULTS: NYHA class significantly improved in the SV compared to the control group (24.9 vs. $6.4 \%$, shifting from class III to II, and 55.4 vs. $25.2 \%$, from class II to I, $\mathrm{p}<0.05$ for all). A significant improvement in LVEF and eGFR levels was found in the SV compared to the control group (42.4 vs. $34.2 \%, 73.8$ vs. $61.2 \mathrm{ml} / \mathrm{min}$, respectively; $\mathrm{p}<0.001$ for all). NT-proBNP, clinic systolic and diastolic $\mathrm{BP}$, blood glucose, HbA1c and UA values were reduced in both treatment arms, but they were lower in the SV compared to the control group (3107 vs. $4552 \mathrm{pg} / \mathrm{mL}, 112.2$ vs. 120.4 and 68.8 vs. $75.6 \mathrm{mmHg}, 108.4$ vs. $112.6 \mathrm{mg} / \mathrm{dL}, 5.4$ vs. $5.9 \%$ and 5.9 vs. $6.4 \mathrm{mg} / \mathrm{dL}$, respectively, p $<0.05$ ). Mortality and re-hospitalization for HF was lower in the SV group (20.1 vs. $33.6 \%$ and 27.7 vs. $46.3 \%$ respectively; $\mathrm{p}<0.05$ for all). No side effects were observed.

CONCLUSIONS: In elderly patients with HFrEF and comorbidities, the treatment with SV seems effective and safe. The improvement in LVEF, BP, eGFR, and metabolic profile could be the mechanisms by which SV play their beneficial role on clinical outcomes.

\section{MYOCARDIAL WORK IN PATIENTS WITH ARTERIAL HYPERTENSION AND DIABETES}

Marijana Tadic ${ }^{1}$, Cesare Cuspidi ${ }^{2}$, Biljana Pencic ${ }^{1}$, Anka Majstorovic ${ }^{1}$, Irena Irena Ilic ${ }^{1}$, Guido Grassi ${ }^{2}$, Vera Celic ${ }^{1}$

${ }^{1}$ University Hospital, Department of Cardiology, Heroja Milana Tepica, Belgrade, Serbia, ${ }^{2}$ Department of Medicine and Surgery, University of Milano-Bicocca, Milan, Italy

AIM: We aimed to investigate myocardial performance using pressure-strain loops in hypertensive patients with and without type 2 diabetes mellitus (DM).

METHODS: This cross-sectional study included 135 subjects (45 controls, 50 hypertensive patients without DM and 40 hypertensive with DM patients) who underwent complete two-dimensional echocardiographic examination (2DE) including two-dimensional speckle-tracking echocardiography. Using pressure-strain curve was used to determine global myocardial work, constructive work, wasted work, and work efficiency in all study participants. RESULTS: Left ventricular (LV) longitudinal and circumferential strains gradually reduced from controls throughout hypertensive subjects to patients with DM and hypertension. Radial strain was lower in patients with concomitant hypertension and DM than in healthy controls. Global myocardial work gradually decreased from controls, throughout hypertensive patients to subjects with hypertension and DM $(2118 \pm 262$ vs. $2021 \pm 228$ vs.
$1913 \pm 255 \mathrm{mmHg} \%, \mathrm{p}=0.001)$. Constructive work also deteriorated in the same direction $(2388 \pm 386$ vs. $2197 \pm 326$ vs. $2081 \pm 347 \mathrm{mmHg} \%, \mathrm{p}<0.001)$. Work efficiency was significantly lower in patients with hypertension and DM in comparison with controls, but there was no difference in comparison to hypertensive patients without DM (97 \pm 3 vs. $92 \pm 3$ vs. $90 \pm 3 \%$, p < 0.001 ). Wasted work did not differ between three observed groups $(95 \pm 53$ vs. $102 \pm 62$ vs. $108 \pm 58 \mathrm{mmHg} \%$, $\mathrm{p}=0.586)$. Glycosylated hemoglobin and systolic blood pressure were associated with LV longitudinal strain and global myocardial work independently of age, body mass index, LV mass and LV ejection fraction in all study participants.

CONCLUSION: Pressure-strain curve showed that myocardial work was significantly affected by hypertension and particularly when hypertension and diabetes were concomitant diseases. Diabetes demonstrated an additional negative effect on myocardial work in hypertensive patients. Glycosylated hemoglobin level and systolic blood pressure were related with myocardial work independently of LV structure and systolic function.

\section{FUNCTIONAL CAPACITY AND LEFT VENTRICULAR DIASTOLIC FUNCTION IN PATIENTS WITH TYPE 2 DIABETES}

\author{
Marijana Tadic ${ }^{1}$, JELENA Suzic-Lazic ${ }^{1}$, VLADAN \\ Vukomanovic ${ }^{1}$, CESARE Cuspidi ${ }^{2}$, SANJA Ilic ${ }^{1}$, \\ VERA Celic ${ }^{1}$
}

${ }^{1}$ University Hospital Department of Cardiology, Heroja Milana Tepica, Belgrade, Serbia, ${ }^{2}$ Department of Medicine and Surgery, University of Milano-Bicocca, Milano, Italy, Milan, Italy

AIM: The study sought to evaluate cardiorespiratory fitness in patients with type 2 diabetes mellitus (DM) with different levels of left ventricular (LV) diastolic dysfunction (LVDD).

Methods: This investigation included 55 controls and 85 uncomplicated diabetic patients, who underwent laboratory analysis, echocardiographic evaluation and cardiopulmonary exercise testing. All DM subjects were separated into 3 groups using the level of LV diastolic function as main criterion: normal, intermediate and LVDD.

RESULTS: Echocardiographic parameters of LV hypertrophy were significantly higher in DM subjects, particularly those with intermediate LV diastolic function and LVDD comparing with controls. The same is valid for parameters of LV diastolic function (E/e', left atrial volume index, and tricuspid regurgitation velocity). Peak 
oxygen uptake was lower, whereas ventilation/carbon dioxide slope was higher, in DM subjects with intermediate LV diastolic function and LVDD in comparison to controls. In the whole study population $\mathrm{HbA} 1 \mathrm{c}, \mathrm{LV}$ mass index and mitral E/e' were independently related with peak oxygen uptake and ventilation/carbon dioxide slope.

CONCLUSION: LVDD significantly impacted functional capacity in DM patients. Glycemic control, LV mass index and LVDD were independently related with peak oxygen consumption and ventilation/carbon dioxide slope in the study population. These results show that timely diagnosis of LVDD and more intensive antidiabetic treatment could prevent target organ damage in DM patients.

\section{CARDIOPULMONARY EXERCISE TESTING AND EXERCISE STRESS ECHOCARDIOGRAPHY: AN INTEGRATED ANALYSIS OF CARDIAC FUNCTION AND EXERCISE CAPACITY IN DYSPNOEIC PATIENTS WITH OR WITHOUT HEART FAILURE}

\author{
Nicolò De Biase ${ }^{1}$, Nicola Riccardo Pugliese ${ }^{1}$, Matteo \\ Mazzola $^{1}$, Luna Gargani ${ }^{2}$, Javier Rosada ${ }^{3}$, Lorenzo \\ Masi $^{3}$, Stefano Taddei ${ }^{1}$, Stefano Masi ${ }^{1}$ \\ ${ }^{1}$ Dipartimento di Medicina Clinica e Sperimentale, \\ Università di Pisa, Pisa; ${ }^{2}$ Istituto di Fisiologia Clinica, \\ CNR, Pisa; ${ }^{3}$ UO Medicina IV, Azienda Ospedaliero- \\ Universitaria Pisana, Pisa
}

INTRODUCTION: Impaired peak oxygen consumption $\left(\mathrm{VO}_{2}\right)$ during exercise is a typical feature of heart failure (HF). This impairment is usually ascribed to alterations in the components of the Fick principle: $\mathrm{VO}_{2}=$ cardiac output (stroke volume $[\mathrm{SV}] \times$ heart rate $[\mathrm{HR}]) \times$ arterio-venous oxygen difference. However, these parameters may not allow for a sufficient characterization of the derangement of cardiopulmonary function in HF.

AIM: We investigated whether echocardiographic indices of cardiac function may relate to peak $\mathrm{VO}_{2}$ independently of the components of the Fick principle.

METHODS: We enrolled 30 healthy controls and 357 dyspnoeic patients: 113 with dyspnoea of unknown origin (DUO: $92 / 113,81 \%$ with arterial hypertension and 72/113, $64 \%$ with diabetes mellitus), 143 with HF with reduced ejection fraction (HFrEF) and 101 with preserved ejection fraction $(\mathrm{HFpEF})$. All subjects underwent an integrated cardiopulmonary exercise testing-exercise stress echocardiography (CPET-ESE). Echocardiographic image acquisition was performed at rest, at anaerobic threshold (AT, i.e. after reaching a stable respiratory exchange ratio $\geq 1.00)$ and at peak effort.
RESULTS: The study population consisted of 387 subjects (mean age: $67.9 \pm 11.1$ years; $68 \%$ males). AT and peak $\mathrm{VO}_{2}$ were significantly impaired in DUO, HFpEF and HFrEF patients. Peak HR was significantly lower in HF patients; rest and peak SV were significantly reduced in $\mathrm{HFrEF}$ patients. In addition to $\mathrm{HR}$ and $\mathrm{SV}$, peak $\mathrm{VO}_{2}$ was significantly and independently related to measures of left ventricular systolic function (Tissue-Doppler Imagingderived S') and left atrial function (LA reservoir strain/E/ e') and to right-sided ventricular-arterial coupling (tricuspid annular plane systolic excursion/systolic pulmonary arterial pressure [TAPSE/sPAP] ratio).

CONCLUSIONS: CPET-ESE revealed multiple abnormalities in cardiac function could co-exist in dyspnoeic patients with and without a definite diagnosis of HF. A thorough evaluation of the pathophysiological background behind dyspnoea may be useful to identify subjects at higher risk of developing HF and develop a tailored medical treatment.

\section{POSSIBLE ADVANTAGES OF THE NEW CLASSIFICATION OF HYPERTENSIVE DISORDERS IN PREGNANCY: TWO CLINICAL CASES}

Chiara Tognola ${ }^{1}$, Giovanni Tavecchia ${ }^{1}$, Milani Martina ${ }^{1}$, Cartella Iside ${ }^{1}$, Sun Jinwey ${ }^{1}$, Grasso Enzo ${ }^{1}$, Alessandro Maloberti ${ }^{1}$, Cristina Giannattasio ${ }^{2}$

${ }^{1}$ Asst Grande Ospedale Metropolitano Niguarda, Milano, Italy, ${ }^{2}$ Universita' Degli Studi Di Milano-Bicocca, Milano, Italy

INTRODUCTION: Hypertensive pregnancy disorders (HDP) complicate $10 \%$ of gestations worldwide. Recently, it has been proposed to reclassify preeclampsia no longer on the basis of gestational age (GA) at which the pressure rise and proteinuria occur, but on the basis of specific maternal hemodynamic parameters in combination with evidence or not of intrauterine growth restriction of the fetus (IUGR).

AIM: We compared two clinical cases of preeclampsia late onset (hypertension + proteinuria $>34 \mathrm{GA}$ ), with and without IUGR.

CASE REPORT: (1) HDP-IUGR 39 years, CVRF: obesity, familiarity with $\mathrm{CHD}$ and $\mathrm{AH}$, first trimester screening: high risk preeclampsia, prophylaxis with ASA. 34 GA: preeclampsia, IUGR, bilateral notches of the uterine arteries. Recovery, treatment with labetalol $100 \mathrm{mg} \times 2$. $36+1$ GA: urgent cesarean section because of CTG's alterations. During the post-partum oliguria and desaturation: thoracic ecoscopy (increased size and thickness of 
$\mathrm{LV}$, systolic function preserved); chest CT diagnostic for APO. Furosemide $20 \mathrm{mg}$ x 7 days + Labetalol $100 \mathrm{mg}$ x $3 \times 10$ weeks. Newborn F, 2080 gr $(<2,5$ pct $)$.

(2) HDP-NIUGR 34 years, no CVRF, no first trimester screening. 34 GA: normal fetal biometry and uterine arteries doppler. 35 GA: preeclampsia, normal fetal growth. Recovery, no therapy. $35+4$ GA: urgent cesarean section because of two incoming eclamptic crisis. Brain $\mathrm{CT}+\mathrm{MRI}$ diagnostics for PRES, ecoscopy (normal size and thickness of LV, EF 63\%). Nifedipine $20 \mathrm{mg} \times 2$ weeks. Newborn F, 2200 gr (25 pct).

It almost seems these two patients suffer from two different pathologies:

(1) HDP-IUGR: maternal cardiovascular disease (same RFs, cardiac dysfunction precedes clinical presentation) with secondary placental hypoxia. Until now, there's evidence of altered LV geometry and myocardial activity, sub-optimal plasmatic expansion (lower SV, CO and HR and higher TVR compared to physiological pregnancy).

(2) HDP-NIUGR: placental disease. Current hemodynamics results are inconclusive ( $\mathrm{CO}$ and TVR similar to physiological pregnancy).

CONCLUSIONS: Our experience confirms these observations. The HDP-IUGR/NIUGR classification provides the basis for a more in-depth pathophysiological knowledge of these disorders, with important clinical consequences in terms of obstetrical-neonatal outcome and lifetime cardiovascular risk.

\section{LEFT VENTRICULAR HYPERTROPHY REGRESSION FOLLOWING RENAL ARTERY REVASCULARIZATION: A META-ANALYSIS}

Cesare Cuspidi ${ }^{\text {ab }}$ MD, Marijana Tadic ${ }^{c}$ Carla Sala ${ }^{\mathrm{d}}$ MD, MD, Fosca Quarti-Trevan ${ }^{\mathrm{a}}$, Elisa Gherbesi ${ }^{\mathrm{d}} \mathrm{MD}$, Giuseppe Mancia ${ }^{\mathrm{a}}$ MD and Guido Grassi ${ }^{\mathrm{a}} \mathrm{MD}$

${ }^{a}$ Department of Medicine and Suurgery, University of Milano-Bicocca, Milano, Italy; ${ }^{b}$ Istituto Auxologico Italiano, Milano, Italy; ${ }^{c}$ University Hospital "Dr. Dragisa Misovic - Dedinje" Department of Cardiology, Belgrade, Serbia., ${ }^{d}$ Department of Clinical Sciences and Community Health, University of Milano and Fondazione Ospedale Maggiore IRCCS Policlinico di Milano, Italy

INTRODUCTION: Few echocardiographic studies have focused on regression of left ventricular hypertrophy (LVH) in patients with renal artery stenosis after revascularization, with inconsistent results.

AIM: We performed a systematic meta-analysis of these studies in order to offer a comprehensive information on this topic.
METHODS: The PubMed, OVID-MEDLINE, and Cochrane library databases were analyzed to search English-language articles published from January 1st 1990 up to March 31st 2020. Studies were identified by crossing the following terms "renal artery stenosis" "reno-vascular hypertension" "fibromuscular dysplasia" "renal artery stenting" "renal artery surgery" with "cardiac damage", "hypertensive heart disease" "left ventricular mass", "left ventricular hypertrophy", "echocardiography" .

RESULTS: A total of 726 hypertensive patients with renal artery stenosis (mean age 61 years, $64 \%$ men, $98 \%$ treated, $10 \%$ with fibromuscular dysplasia) were included in 13 studies. Baseline and post-intervention pooled mean LV mass versus baseline values were $203 \pm 19 \mathrm{~g}$ and $220 \pm 15 \mathrm{~g}$, respectively (SMD $-0.24 \pm 0.06$, CI $-0.37 /$ $0.21, \mathrm{p}<0.0001$ ); corresponding values for LV mass index were $115 \pm 7 \mathrm{~g} / \mathrm{m} 2$ and $129.0 \pm 6 \mathrm{~g} / \mathrm{m} 2$, respectively (SMD $-0.28+0.04$, CI $-0.36-0.21, \mathrm{p}<0.0001$ ). Renal revascularization was associated with a $40 \%$ lower risk of LVH. This trend was accompanied by a reduction in the number of antihypertensive drugs (SMD $-0.27+0.04$, CI 0.37-0.17, $\mathrm{p}<0.0001$ ).

CONCLUSIONS: The present meta-analysis suggests that renal artery revascularization added to anti-hypertensive therapy promotes a favourable effect on LV structure, as reflected by a significant decrease in absolute and indexed LV mass index as well by a lower risk of LVH. Limitations include :I) high prevalence of modest renal artery stenosis (> 50\%); II) small sample of fibromuscular dysplasia; III) lack of randomized design of most studies.

\section{CARFILZOMIB THERAPY IMPAIRS LEFT VENTRICULAR SYSTOLIC AND DIASTOLIC FUNCTION IN MULTIPLE MYELOMA PATIENTS}

Mingrone $\mathrm{G}^{1}$, Astarita $\mathrm{A}^{1}$, Maffei $\mathrm{I}^{1}$, Leone $\mathrm{D}^{1}$, Catarinella $\mathrm{C}^{1}$, Cesareo $\mathrm{M}^{1}$, Airale $\mathrm{L}^{1}$, Salvini $\mathrm{M}^{2}$, Gay $\mathrm{F}^{2}$, Bringhen $\mathrm{S}^{2}$, Vallelonga $\mathrm{F}^{1}$, Veglio $\mathrm{F}^{1}$, Milan $\mathrm{A}^{1}$

${ }^{1}$ Hypertension Unit, Department of Medical Sciences, University of Turin, "Città della Salute e della Scienza" Hospital, Turin, Italy; ${ }^{2}$ Myeloma Unit, Division of Haematology, University of Turin, "Città della Salute e della Scienza” Hospital, Turin, Italy

INTRODUCTION: Carfilzomib improves the prognosis of multiple myeloma (MM) patients, but significantly increases cardiovascular toxicity. The timing and effect of Carfilzomib therapy on left ventricular function is still under investigation.

AIM: We sought to assess the echocardiographic systodiastolic changes, including global longitudinal strain 
(GLS), in patients treated with Carfilzomib and to identify predictors of increased risk of cardiovascular adverse events (CVAEs) during therapy.

METHODS: 88 patients with MM performed a baseline cardiovascular evaluation comprehensive of transthoracic echocardiogram (TTE) before the start of Carfilzomib therapy and after about 6 months. All patients were followed-up to early identify the occurrence of CVAEs during therapy.

RESULTS: After Carfilzomib treatment, mean GLS slightly decreased $(-22.2 \% \pm 2.6$ vs $-21.3 \% \pm 2.5$; $\mathrm{p}<0.001) .58 \%$ of patients experienced CVAEs during therapy: $71 \%$ of them had uncontrolled arterial hypertension, 29\% had major CVAEs or CV events not related to arterial hypertension. GLS variation during therapy was not related to an increased risk of CVAEs; however, patients with baseline GLS $\geq-21 \%$ and/or left ventricular ejection fraction $\leq 60 \%$ had an increased risk of major CVAEs ( $\mathrm{p}=0.004, \mathrm{OR}=6.2 ; \mathrm{p}=0.04$, OR $=3.7$; respectively). Carfilzomib led to an increased risk of diastolic dysfunction $(5.6 \%$ vs $13.4 \% \mathrm{p}=0.04)$ and to a rise in $\mathrm{E} / \mathrm{e}$ ' $(8.9 \pm 2.7$ vs $9.7 \pm 3.7 ; \mathrm{p}=0.006)$.

CONCLUSIONS: Carfilzomib leads to LV function impairment early demonstrated by GLS changes and diastolic dysfunction. Baseline echocardiographic evaluation, through the estimation of GLS and LVEF, might improve cardiovascular risk stratification before treatment.

\section{ROLE OF MACROPHAGES IN CARDIAC DAMAGE INDUCED BY DOXORUBICIN}

\author{
Jessica Gambardella ${ }^{1}$, Antonella Fiordelisi ${ }^{1}$, Nella \\ Prevete $^{2}$, Fabrizio Dal Piaz ${ }^{3}$, Michele Ciccarelli $^{3}$, \\ Guido Iaccarino $^{1}$, Daniela Sorriento ${ }^{1}$
}

${ }^{1}$ Dipartimento di Scienze Biomediche Avanzate, Università Federico II di Napoli; ${ }^{2}$ Dipartimento di Scienze Mediche Traslazionali, Università Federico II di Napoli; ${ }^{3}$

Dipartimento di Medicina, Chirurgia e Odontoiatria

"Scuola Medica Salernitana"/DIPMED, Università degli Studi di Salerno

INTRODUCTION: Anthracyclines, such as Doxorubicin (DOX), are effective anti-cancer drugs whose use is limited by cumulative dose-dependent cardiotoxicity. Even if cardiomyocytes are the most common target of research studies on cardiotoxicity, the functional contribution of non-myocyte cardiac cells, such as inflammatory cells, is recently becoming of growing interest.

AIM: to evaluate the role of inflammation in DOX-dependent cardiac damage.
METHODS: C57/B16 mice were treated with DOX (10 $\mathrm{mg} / \mathrm{Kg}$ ) by intraperitoneal injection and hearts were collected after 5 days to perform histological analysis. In hearts treated with DOX, the histological analysis showed a significant macrophages infiltration. In order to evaluate whether a crosstalk could exist between macrophages and cardiac cells that could affect cardiac outcomes in response to DOX, we compared the effects of two different treatments in cardiomyoblasts (H9C2): incubation with culture medium from macrophages (RAW 264.7) treated with DOX for $24 \mathrm{~h}$ (M-DOX) and direct treatment with DOX (D-DOX).

RESULTS: Both conditions induced an increase of Cleaved caspase-3 levels that was higher in response to M-DOX. Since apoptosis in response to DOX is mediated by p53, we evaluated p53 levels by western blot. In response to $\mathrm{M}-\mathrm{DOX}$, p53 is accumulated in mitochondria where it induced the release of cytochrome c, suggesting that p53-dependent mitochondrial damage occurs in response to M-DOX. Accordingly, mitochondrial morphology, evaluated by fluorescence microscopy analysis of Mitotracker staining, was altered. This was associated with an impaired autophagic flux, as demonstrated by levels of LC3-II and accumulation of autophagosomes.

CONCLUSIONS: All these data clearly indicate that in vivo doxorubicin induces macrophage infiltration that could potentially contribute to cardiac cell damage. In vitro, the conditioned medium from DOX-activated macrophages amplifies cardiomyoblasts damage by inhibiting autophagy and enhancing p53 mediated mitochondrial apoptosis. It is likely that macrophages release cytokines which activate p53 enhancing DOXO dependent cardiac damage.

\section{CARDIAC REMODELING INDUCED BY PRESSURE OVERLOAD ACTIVATES} A NEUROIMMUNE MECHANISM IN THE SPLEEN WHICH THROUGH P1GF INDUCES THE RECRUITMENT OF ADAPTIVE MACROPHAGES INTO THE LEFT CARDIAC VENTRICLE

${ }^{1}$ Perrotta Sara, ${ }^{2}$ Carnevale Raimondo, ${ }^{2}$ Pallante Fabio, ${ }^{2}$ Mastroiacovo Francesco, ${ }^{2}$ Cifelli Giuseppe, ${ }^{1,2}$ Lembo Giuseppe, ${ }^{1,2}$ Carnevale Daniela

${ }^{1}$ Department of Molecular Medicine, Sapienza University of Rome, Italy; ${ }^{2}$ Department of Angiocardioneurology and Translational Medicine, IRCCS Neuromed, Pozzilli, Italy

INTRODUCTION: Cardiac remodeling induced by pressure overload is the process established by the left ventricle 
(LV) which involves immune cells. We previously found that placenta-derived growth factor (P1GF), a growth factor belonging to the VEGF family, is necessary for adaptive remodeling to pressure overload.

AIM: we investigate the immuno-modulator role of PIGF in response to pressure overload induced by coarctation of the aorta (TAC) in mice.

METHODS: We analyzed cardiac monocyte/macrophage infiltration by flow cytometry to discriminate the resident response from the recruited one using CD11b/CD64/ Timd4/Ly6C markers, in mice PIGF KO and WT at 4 days from $\mathrm{TAC} / \mathrm{sham}$ procedure.

RESULTS: WT mice, but not PlGF KO, showed a significant infiltration of non-resident macrophages after TAC, suggesting that PIGF may influence their recruitment. Given that the expression of PIGF was increased after TAC in the heart and spleen, we generated chimeric mice through spleen transplantation between WT and PIGF KO. PlGF KO mice with WT spleen showed a typical adaptive remodeling to TAC, as evidenced by echocardiographic analysis. In contrast, WT mice with PIGF KO spleen showed an early heart failure (HFrEF) response after TAC. To investigate the potential role of the spleen as a reserve of myeloid cells with adaptive/reparative functions, we splenectomized mice observing an early HFrEF and a reduced monocyte/macrophage recruitment in LV. To study the involvement of a neuroimmune mechanism, we performed a selective denervation of splanchnic district through the surgical removal of the celiac ganglion (CGX). Mice subjected to CGX and TAC showed a HFrEF and a reduced recruitment of monocytes/macrophages in LV similarly to splenectomized and PIGF KO mice.

CONCLUSIONS: These data demonstrate that pressure overload activates a neuroimmune mechanism in the spleen that induces the PIGF release as an effective immunomodulator to recruit adaptive/reparative macrophages in the heart promoting LV adaptive remodeling to pressure overload.

\section{ATRIAL VOLUME INDEXED BY HEIGHT AS A NEW MARKER FOR HYPERTENSION- MEDIATED ORGAN DAMAGE IN FEMALE POPULATION}

Lorenzo Airale 1, Anna Paini 2, Eugenia Iannello 3, Costantino Mancusi 4, Antonella Moreo 5, Gaetano Vaudo 6, Eleonora Avenatti 1, Massimo Salvetti 2, Stefano Bacchelli 3, Raffaele Izzo 4, Paola Sormani 5, Alessio Arrivi 6, Maria Lorenza Muiesan 2, Daniela Degli Esposti 3, Cristina Giannattasio 5, Giacomo Pucci 6, Nicola De Luca 4, Alberto Milan 1
1 Dpt of Medical Sciences, Hypertension Center, University of Torino, Torino, ITALY, 2 Department of Internal Medicine, ASST Spedali Civili di Brescia, Brescia, ITALY, 3 Cardiothoracic and Vascular Department, S. Orsola Malpighi University Hospital, Bologna, ITALY, 4 Hypertension Research Center, Federico II University Hospital, Napoli, ITALY, 5 Echo Lab, Cardiologia IV, De Gasperis Dep, ASST Niguarda Ca granda e Dip Medicina e Chirurgia, Università Milano Bicocca, Milano, ITALY, 6 f SC Medicina Interna, Azienda Ospedaliero Universitaria di Terni, Terni, ITALY

INTRODUCTION: Left Atrial enlargement (LAe) is a subclinical marker of hypertensive mediated organ damage, whose identification is important in cardiovascular risk stratification. Recently, LA indexing for height has been suggested as more accurate marker to define LAe.

AIM: to test the difference in LAe prevalence using Body surface area (BSA) and height ${ }^{2}$ definitions in an essential hypertensive population.

METHODS: A total of 441 essential hypertensive patients underwent complete clinical and echocardiographic evaluation. Left Atrial Volume (LAV), left ventricular morphology and sisto-diastolic function were evaluated.

RESULTS: LAe was twice as prevalent when defined using height ${ }^{2}\left(\mathrm{LAe}_{\mathrm{h} 2}\right)$ indexation rather than BSA $\left(\mathrm{LAe}_{\mathrm{BSA}}\right) \quad(51 \% \mathrm{vs} .23 \%, \mathrm{p}<0.001) . \mathrm{LAe}_{\mathrm{h} 2}$, but no $\mathrm{LAe}_{\mathrm{BSA}}$, was more prevalent in females $(\mathrm{p}<0.001)$. Male and female differed also for Left Ventricular Hypertrophy $(p=0.046)$ and Left Ventricular Diastolic Disfunction (LVDD) indexes (septal Em/Etdi: $\mathrm{p}=0.009$; lateral $\mathrm{Em} /$ Etdi: $p=0.003$; mean Em/Etdi: $p<0.002$ ). All patients presenting $\mathrm{LAe}_{\mathrm{BSA}}$ also met criteria for $\mathrm{LAe}_{\mathrm{h} 2}$. According to presence-absence of LAe, we created 3 groups $\left(\right.$ Norm $=$ BSA- $/ \mathrm{h}^{2}-; \quad$ DilH $=\mathrm{BSA}-/ \mathrm{h}^{2}+; \quad$ DilHB $=$ BSA $+/ h^{2}+$ ). Female sex prevalence in DilH group was higher than in both others (Norm: $p<0.001$; DilHB: $\mathrm{p}=0.036)$. LVH and mean and septal Em/Etdi grew from Norm to DilH group and from DilH to DilHB group ( $p<0.05$ for all comparisons).

CONCLUSIONS: $\mathrm{LAe}_{\mathrm{h} 2}$ identified twice as many patients as having LAe compared to $\mathrm{LAe}_{\mathrm{BSA}}$. Both $\mathrm{LAe}_{\mathrm{h} 2}$ and $\mathrm{LAe}_{\mathrm{BSA}}$ definitions are associated with LVH and LVDD. In female patients, $\mathrm{LAe}_{\mathrm{h} 2}$ definition and its sex-specific threshold seems to be more sensible than $\mathrm{LAe}_{\mathrm{BSA}}$ in identifying a chamber enlargement. 


\section{CARFILZOMIB THERAPY IN MULTIPLE MYELOMA: CARDIOVASCULAR ADVERSE EVENTS AND CARDIOVASCULAR RISK ASSESSMENT}

Anna ASTARITA ${ }^{\mathbf{a}}$, Giulia MINGRONE ${ }^{\mathbf{a}}$, Lorenzo AIRALE $^{\mathrm{a}}$, Fabrizio VALLELONGA ${ }^{\mathrm{a}}$, Cinzia CATARINELLA $^{\text {a }}$, Marco CESAREO ${ }^{\mathbf{a}}$, Dario LEONE $^{\mathbf{a}}$, Giulia BRUNO ${ }^{\mathbf{a}}$, Ilaria MAFFEI ${ }^{\mathbf{a}}$, Franco VEGLIO $^{\mathrm{a}}$, Alberto MILAN ${ }^{\mathrm{a}}$

${ }^{a}$ Department of Medical Sciences, Division of Internal Medicine, Hypertension Unit, AO "Città della Salute e della Scienza" University Hospital, Turin, Italy

INTRODUCTION: Cardiovascular adverse events (CVAEs) are closely related to Carfilzomib (CFZ) therapy in multiple myeloma (MM), but their prevention is challenging due to lack of validated predictors of cardiovascular events. Moreover, the nature and prevalence of each CVAEs is debated, due to the lack of 'real-life' perspective trials.

AIM: We sought to identify predictors for CVAEs in patients ongoing Carfilzomib therapy. For the first time, the nature and the incidence of each CVAEs in a 'real-life' study were assessed.

METHODS: MM patients with indication to CFZ therapy were prospectively enrolled. A comprehensive baseline evaluation, previously CFZ beginning, were performed: cardiovascular anamnesis, office blood pressure (BP), ABPM, ECG, trans-thoracic Echocardiography, pulse wave velocity (PWV). After starting the CFZ treatment, the incidence of CVAEs was detected.

RESULTS: 116 patients were enrolled, between 2015 and 2020. At basal evaluation, the proportion of target organ damage and uncontrolled hypertension $(51.7 \%)$ were substantial. Main predictors of CVAEs were: systolic hypertension office $(\beta$ 4.09; $\mathrm{p}=0.001)$ and day $(\beta$ 2.27; $\mathrm{p}=0.044)$, blood pressure variability $\geq 10 \quad(\beta$ 5.07; $\mathrm{p}=0.002)$, left ventricular hypertrophy $(\beta$ 3.06; $\mathrm{p}=0.021), \quad \mathrm{PWV} \geq 8.75 \mathrm{~m} / \mathrm{s}(\beta$ 5.13; $\mathrm{p}=0.000)$ and global longitudinal strain $(\beta 1.19 ; \mathrm{p}=0.003)$. During a follow-up of $13.56 \pm 9.63$ months after CFZ starting, the rate of all-grade CVAEs was $44.8 \%$, distinct in hypertensive events $(30.2 \%)$ and major (14.7\%). The chronic rise/ new onset of arterial hypertension $(31.9 \%)$ and uncontrolled hypertension pre-CFZ infusion $(22.4 \%)$ were the most frequent hypertensive events, arrhythmias (41.2\%) and acute coronary syndromes $(23.5 \%)$ the most frequent within the major CVAEs; one patient died of sudden cardiac arrest.

CONCLUSIONS: CFZ therapy is linked to high rate of CVAEs, potentially life-threatening. A comprehensive cardiovascular evaluation is effective in CVAEs prediction and need to allow risk mitigation strategies.

\section{ARTERIAL HYPERTENSION IN SYSTEMIC SCLEROSIS: EFFECTS ON DIASTOLIC DYSFUNCTION}

Matteo Mazzola1-2, Nicola Riccardo Pugliese2, Nicolò

De Biase2, Gennaro D'Angelo1, Cosimo Bruni3, Marco Matucci-Cerinic3, Stefano Masi2, Stefano Taddei2, Luna Gargani1

${ }^{1}$ Institute of Clinical Physiology-C.N.R., Pisa, Italy; ${ }^{2}$ Department of Clinical and Experimental Medicine, University of Pisa, Pisa, Italy;: ${ }^{3}$ Department of Experimental and Clinical Medicine, Division of Internal Medicine and Rheumatology, Azienda Ospedaliera Universitaria Careggi, Florence, Italy

INTRODUCTION: Both arterial hypertension (HT) and Systemic Sclerosis (SSc) are responsible for a progressive myocardial involvement leading to cardiac remodeling and diastolic dysfunction. Nevertheless, little is known about how these conditions interact.

METHODS: We enrolled 277 SSc patients with (94 pts, SSc/HT) and without (183 pts, SSc) HT. Patients with pulmonary artery hypertension (PAH) were excluded from the study. All patients underwent a complete transthoracic echocardiography comprehensive of diastolic evaluation according to European Recommendations.

RESULTS: Patients from the two groups were age-matched and displayed a similar global systolic function in terms of ejection fraction and systolic Tissue Doppler Imaging (TDI). Compared to SSc group all the main diastolic parameters were worse in SSc/HT except for systolic pulmonary arterial pressure which is likely to be elevated in these patients mainly as a result of the increase in pulmonary vascular resistance. Interventricular septum and left ventricular posterior wall thickness of SSc/HT patients were also higher than SSc. Furthermore, patients with SSc/HT were more commonly treated with dihydropiridinic calcium channel blockers (CCB) [CCB SSc: $26(14.2 \%)$ vs CCB SSc/HT: 24 (25.5\%) vs, p = 0.031], renin-angiotensin-aldosterone system inhibitors (RAASI) 
[RAASI SSc: 7 (3.8\%) vs RAASI SSc/HT: 50 (53.2\%), $\mathrm{p}<0.0001]$ and beta-blockers (BB) [BB SSc: 3 (1.6) vs BB SSc/HT: $10(10,6 \%), p=0.001]$.

\begin{tabular}{|l|l|l|l|}
\hline & $\begin{array}{l}\text { SSc } \\
\text { (n=183) }\end{array}$ & $\begin{array}{l}\text { SSc/HT } \\
(\mathbf{n}=94)\end{array}$ & $p$ \\
\hline Age (years) & $61.3 \pm 9.0$ & $62.2 \pm 11.1$ & 0.48 \\
\hline LVEDD (mm) & $43.8 \pm 4.3$ & $44.1 \pm 4.5$ & 0.59 \\
\hline Ejection Fraction (\%) & $64.0 \pm 6.1$ & $64.0 \pm 5.9$ & 0.98 \\
\hline TDI S' & $8.6 \pm 1.3$ & $8.7 \pm 2.1$ & 0.76 \\
\hline $\begin{array}{l}\text { Interventricular } \\
\text { Spetum (mm) }\end{array}$ & $9.4 \pm 1.5$ & $10.3 \pm 1.7$ & $<0.0001$ \\
\hline $\begin{array}{l}\text { Posterior } \\
\text { Wall (mm) }\end{array}$ & $8.6 \pm 1.3$ & $9.3 \pm 1.4$ & $<0.001$ \\
\hline $\begin{array}{l}\text { Left Atrial } \\
\text { Volume (ml/mq) }\end{array}$ & $44 \pm 14$ & $49 \pm 15$ & $\mathbf{0 . 0 4}$ \\
\hline E/e' & $8.1 \pm 2.5$ & $9.0 \pm 3.2$ & $\mathbf{0 . 0 3}$ \\
\hline $\begin{array}{l}\text { Pulmonary Artery Systolic Pressure (mmHg) } \\
\text { (mm }\end{array}$ & $29 \pm 7$ & $29 \pm 8$ & 0.60 \\
\hline
\end{tabular}

CONCLUSIONS: Both SSc and HT can affect left ventricular diastolic function. Patients with SSc and HT display worse echocardiographic parameters of diastolic dysfunction compared to patients with isolated SSc, despite the more frequent treatment with vasoactive drugs. Being a further source of diastolic pattern impairment, the presence of HT should be considered in the echocardiographic evaluation of SSc patients.

\section{URIC ACID IN ACUTE CORONARY SYNDROMES: CORRELATION WITH IN-HOSPITAL MORTALITY AND COMPLICATIONS}

Martina Milani ${ }^{1}$, Alessandro Maloberti ${ }^{1}$, Iside Maria Cartella $^{1}$, Chiara Tognola ${ }^{1}$, Enzo Grasso ${ }^{1}$, Giovanni Amedeo Tavecchia ${ }^{1}$, Marco Biolcati ${ }^{1}$, Valentina Giani $^{1}$, Sofia Bianchi ${ }^{1}$, Paola Rebora ${ }^{2}$, Nuccia Morici ${ }^{3}$, Alice Sacco ${ }^{3}$, Giovanna Viola ${ }^{3}$, Francesca Lattuada ${ }^{1}$, Valentina Molinari ${ }^{1}$, Lucia Occhi ${ }^{1}$, Ilaria Bassi ${ }^{1}$, Andrea Portoghese ${ }^{1}$, Matteo Fortuna ${ }^{1}$, Fabrizio Oliva ${ }^{3}$, Cristina Giannattasio ${ }^{1,4}$

1- School of Medicine and Surgery, University of MilanoBicocca, Monza, Italy; 2- Center of Biostatistics for Clinical Epidemiology, School of Medicine and Surgery, University of Milano-Bicocca, Milan, Italy; 3- Cardiology I, "A.De Gasperis” Department, ASST GOM Niguarda Ca' Granda, Milan, Italy; 4-Cardiology IV, “A.De Gasperis" Department, ASST GOM Niguarda Ca' Granda, Milan, Italy

INTRODUCTION: Uric acid (UA) has been related to inhospital mortality in patients with acute coronary syndromes (ACS), to early relapse, after ACS, of non-fatal cardiovascular events and to negative intermediate outcomes such as use of intra-aortic balloon pump, non-invasive ventilation, longer inward stay, bleeding, clinical presentation with Atrial Fibrillation (AF) or Heart Failure (HF).

AIM: to evaluate the role of UA as a possible determinant of primary (in-hospital mortality) and secondary outcomes defined as variables of relapsing ischemia (myocardial reinfarction, in-stent thrombosis, bleeding, stroke), worse clinical presentation (with $\mathrm{HF}$ or AF, admission EF, trivasal coronary artery disease (CAD) at the coronary angiography), in-hospital complications (use of inotropes, intra-aortic balloon pump and non-invasive ventilation during hospital stay) and worse recovery (discharge EF).

METHODS: 563 patients, admitted for ACS to Cardiological Intensive Care Unit of Niguarda Ca' Granda Hospital, were enrolled for this retrospective cohort study. Cox regression analysis was performed to evaluate the association between UA and primary and secondary outcomes, adjusting for the following covariates: age, gender, previous myocardial infarction, arterial hypertension, Charlson Comorbidity Index and creatinine.

RESULTS: mean age was $66.5 \pm 12.3$ years, $79.2 \%$ of the patients were males and $49.9 \%$ were ACS-STEMI. Hyperuricemic subjects were older, with more prominent cardiovascular risk factors and history of previous myocardial infarction. They more frequently died during hospital stay, had HF and AF as clinical presentation, more commonly had trivasal CAD and needed intra-aortic balloon pump and non-invasive ventilation. Also EF at admission and discharge were lower in hyperuricemic patients. At multivariate analysis, UA was a significant determinant of primary and secondary outcomes (except for trivasal CAD, bleeding, stroke, re-infaction and in-stent thrombosis).

CONCLUSIONS: UA is an independent determinant of in-hospital mortality and a variable suggestive for worse clinical presentation, in-hospital complications and worse recovery. 
PREDICTING HEART FAILURE TRANSITION AND PROGRESSION: A WEIGHTED RISK SCORE FROM BIO-HUMORAL, CARDIOPULMONARY AND ECHOCARDIOGRAPHIC STRESS TESTING

Nicola Riccardo Pugliese ${ }^{1}$, Nicolò De Biase ${ }^{1}$, Lorenzo Conte $^{2}$, Luna Gargani ${ }^{3}$, Matteo Mazzola ${ }^{1}$, Iacopo Fabiani $^{4}$, Andrea Natali ${ }^{1}$, Frank L. Dini ${ }^{1}$, Paolo Frumento $^{5}$, Javier Rosada ${ }^{6}$, Stefano Taddei ${ }^{1}$, Barry A. Borlaug $^{7}$, Stefano Masi ${ }^{1}$

${ }^{1}$ Department of Clinical and Experimental Medicine, University of Pisa, Pisa, Italy; ${ }^{2}$ Cardiology Unit, Ospedale Castelnuovo Garfagnana, Italy; ${ }^{3}$ Institute of Clinical Physiology-C.N.R., Pisa, Italy; ${ }^{4}$ Fondazione Toscana G. Monasterio, Pisa, Italy; ${ }^{5}$ Department of Political Sciences, University of Pisa, Pisa, Italy; ${ }^{6}$ Fourth Unit of Internal Medicine, University Hospital of Pisa, Pisa, Italy;

${ }^{7}$ Division of Cardiovascular Diseases, Department of Medicine, Mayo Clinic, Rochester, Minnesota

AIM: We tested the prognostic role of a risk score including bio-humoral evaluation, cardiopulmonaryechocardiographic stress (CPET-ESE) and lung ultrasound, in patients with heart failure (HF) with reduced and preserved ejection fraction (HFrEF and HFpEF), and subjects at risk of developing HF (American College of Cardiology/ American Heart Association Stages A and B).

METHODS: We evaluated 318 subjects: 94 in Stages A-B, 194 in Stage C (85 HFpEF and 109 HFrEF), and 30 age and sex-matched controls (Stage 0). During a median follow-up of 18.5 months, we reported 40 urgent HF visits, $31 \mathrm{HF}$ hospitalisations and 10 cardiovascular deaths. Cox proportional-hazards regression for predicting adverse events identified five independent predictors and each was assigned a number of points proportional to its regression coefficient: $\Delta$ stress-rest B-lines $>10$ (3 points), peak oxygen consumption $<16 \mathrm{~mL} / \mathrm{kg} / \mathrm{min}$ ( 2 points), minute ventilation/carbon dioxide production slope $\geq 36 \quad(2$ points), peak systolic pulmonary artery pressure $\geq 50$ $\mathrm{mmHg}$ (1 point) and resting N-terminal pro-brain natriuretic peptide (NT-proBNP) $>900 \mathrm{pg} / \mathrm{mL}$ (1 point). We defined three risk categories: low-risk $(<3$ points $)$, intermediate-risk (3-6 points), and high-risk ( $>6$ points).

RESULTS: The event-free survival probability for these three groups were 93\%, 52\% and 20\%, respectively. Hazard Ratio was 4.55 for each risk category upgrade $(95 \%$ confidence interval [CI] 3.44-5.93). The area-under-curve for the scoring system to predict events was 0.92 (95\% CI 0.88-0.96).

CONCLUSIONS: A multiparametric risk score including indices of exercise-induced pulmonary congestion, markers of cardiopulmonary dysfunction and NT-proBNP identifies patients at increased risk for $\mathrm{HF}$ events across the HF spectrum.

\section{ATRIAL FIBRILLATION AND CARDIOVASCULAR DEATH IN GENERAL POPULATIONS: SYSTEMATIC REVIEW AND META-ANALYSIS}

Francesca Coccina ${ }^{1}$, Anna M. Pierdomenico ${ }^{1}$, Angelo

Di Censo ${ }^{1}$, Jacopo Pizzicannella ${ }^{1}$, Rosalinda

Madonna $^{2}$, Maria T. Guagnano ${ }^{1}$, Oriana Trubiani ${ }^{1}$, Francesco Cipollone $^{1}$, Sante D. Pierdomenico ${ }^{1}$

${ }^{1}$ Università degli Studi Gabriele d'Annunzio, ChietiPescara, Chieti ${ }^{2}$ Università degli Studi di Pisa, Pisa

INTRODUCTION: The association between atrial fibrillation and cardiovascular death is not yet clear. Moreover, recent meta-analyses on the association between atrial fibrillation and cardiovascular death assessed selected populations and did not exclude stroke death.

AIM: to perform a systematic review and meta-analysis on the association between atrial fibrillation and cardiovascular death, including and excluding stroke death, in general populations.

METHODS: We identified 11 eligible studies. Separate data for men and women or type of event were reported in some studies, so we could obtain 17 subgroups. Studies included 1408290 subjects experiencing more than 8000 events. Data were pooled together by random effects model.

RESULTS: The overall adjusted hazard ratio and 95\% confidence interval for cardiovascular death was 2.85 (2.33-3.49) in patients with versus those without atrial fibrillation. Subgroup meta-analysis showed that hazard ratio and 95\% confidence interval was $2.37(1.63-3.47)$ in men and 3.73 (2.59-5.37) in women, 3.18 (2.47-4.10) for cardiovascular death including stroke death and 2.55 (1.94-3.37) for cardiac death (coronary disease, heart failure and sudden cardiac death). Risk was similar by follow-up length, ethnicity and study design.

CONCLUSIONS: In general populations, patients with versus those without atrial fibrillation had approximately 3 times higher risk of cardiovascular death and 2.5 times 
higher risk of cardiac death. Risk tended to be higher in women than in men.

\section{CLINICAL AND ECHOCARDIOGRAPHIC FEATURES OF PATIENTS AFFECTED BY HEART FAILURE WITH MID-RANGE EJECTION FRACTION COMPARED TO REDUCED AND PRESERVED}

Marco Biolcati ${ }^{1}$, Alessandro Maloberti ${ }^{1,2}$, Claudia Pellegrinelli ${ }^{1}$, Umberto Ceratti ${ }^{1,2}$, Oriana Belli ${ }^{1,2}$, Francesca Casadei ${ }^{1,2}$, Francesco Musca ${ }^{1,2}$, Paola Sormani $^{1,2}$, Gloria Santambrogio ${ }^{1,2}$, Francesca Spanò $^{1,2}$, Benedetta De Chiara ${ }^{1,2}$, Antonella Moreo ${ }^{1,2}$, Cristina Giannattasio ${ }^{1,2}$

1- School of Medicine and Surgery, Milano-Bicocca University, Milan, Italy; 2 - Cardiology IV, "A. De Gasperis" Department, ASST Grande Ospedale Metropolitano Niguarda Ca' Granda, Milan, Italy

INTRODUCTION: The 2016 ESC guidelines classify Heart Failure (HF) according to levels of Ejection Fraction (EF) in $\mathrm{HF}$ with reduced $\mathrm{EF}$ ( $\mathrm{HFrEF}, \mathrm{EF}<40 \%$ ), preserved ( $\mathrm{HFpEF}, \mathrm{EF} \geq 50 \%$ ) and the newborn mid-range (HFmrEF, EF 40-49\%).

AIM: to analyse clinical and echocardiographic features of patients hospitalized for HF when divided accordingly to their admission EF, focusing on HFmrEF.

METHODS: We enrolled 192 patients hospitalized in the Internal Medicine of our hospital between January and September 2017. We collected data on clinical history, P.E., laboratory tests, pharmacological treatment and echocardiography; follow-up for subsequent fatal and nonfatal events ended in May 2018.

RESULTS: Prevalence of HFpEF (55.21\%) is higher than HFrEF (23.60\%) and HFmrEF (17.19\%). Mean age is $80.9 \pm 8.3$ years, and does not differ among groups. HFpEF are more commonly female, show higher SBP at the presentation $(\mathrm{p}<0.001)$ and have more non-cardiac comorbidities, such as renal dysfunction $(\mathrm{p}<0.001)$, anemia $(p=0.05)$, COPD $(p=0.036)$ and also AF $(p=0.04)$; in this group hypertensive aetiology is significantly prevailing $(\mathrm{p}=0.002)$. On the contrary previous myocardial ischaemia and higher NT-proBNP levels on the admission $(p=0.038)$ are typical in HFrEF. Diastolic and systolic dysfunctions co-exist in patients and many candidates show marks of inverse remodelling. HFmrEF carry intermediate attributes (prevalence of CAD and systemic multimorbidities) and exhibit a 'hybrid' US hypertrophy pattern (high RWT, medium LVMi); compared to the other groups, they include the lowest trend on NYHA classification ( $\mathrm{I}-\mathrm{II}, \mathrm{p}=0.027$ ), a less aggressive treatment with the shortest number of drugs and dosages $(p=0.04)$ and show decreased mortality and rehospitalization rates. During the follow-up, patients with HFmrEF experience more longitudinal transition among groups (33\% becomes $\mathrm{HFpEF}$ and 23\% HFrEF).

CONCLUSIONS: HFmrEF share features of both HFrEF and HFpEF, with a high prevalence of CAD and non-cardiac comorbidities, and a large longitudinal transition to the other two classes.

\section{GRK2 EXPRESSION IN IMMUNE CELLS PREDICTS CARDIAC SYSTOLIC FUNCTION AFTER STEMI}

Elena De Angelis, Mariarosaria Rusciano, Maria Grazia Petti, Carmine Vecchione, Gennaro Galasso, Michele Ciccarelli

\section{Department of Medicine and Surgery-Division} of Cardiology- University of Salerno

INTRODUCTION: Cardiac remodeling after an acute myocardial infarct is characterized by molecular, cellular, and interstitial changes leading to changes in size, shape, and function of the heart. Inflammation and immune responses play a crucial role in infarct healing and subsequent LV remodeling. In the setting of heart failure (HF) the observed increased level and activity of GRK2 is correlated to harmful effects on cardiac function and a worse prognosis. GRK2 levels and activity in peripheral blood mononuclear cells (PBMC) are increased in patients with acute myocardial ischemia and associated with a poor prognosis. The possible role of GRK2 in the regulation of the immune system during AMI and involvement in the following cardiac remodeling is not known.

METHODS: We enrolled 49 patients with the diagnosis of STEMI. Levels of GRK2 were evaluated in peripheral blood mononuclear cells (lymphocytes, monocytes, and granulocytes), by FACS analysis at hospital admission (time 0 ) and 24 and $96 \mathrm{~h}$ post-admission.

RESULTS: At the admission, all patients showed a depressed cardiac systolic function as estimated by an $\mathrm{EF}<40 \%$. After five days post-admission $53.85(\mathrm{n}=31)$ displayed a recovering of cardiac systolic function (EF $>$ $45 \%)$ while $46,15 \%(n=18)$ did not show a significant recover of systolic function $(\mathrm{EF}<45 \%)$. At time 0 , white blood cells (monocytes, lymphocytes, and granulocytes) from patients with $\mathrm{EF}<45 \%$ (Fig 1), showed an increased level of GRK2 expression compared to the EF $>45 \%$ group (lymphocytes: $73.4 \%$ vs $11.3 \%$, monocytes: $73.5 \%$ vs $3 \%$, granulocytes $9.4 \%$ vs $2.3 \%$ ) (Fig 2). The levels of 
GRK2 in EF $<45 \%$ group were persistently higher also at 48 and $96 \mathrm{~h}$ post-admission. Interestingly, in the group with $\mathrm{EF}>45 \%$, the level of GRK2 in lymphocytes and monocytes increased at $96 \mathrm{~h}$ post-admission respect to 0 and $48 \mathrm{~h}$ post-admission.

CONCLUSIONS: Our data demonstrate that the different patterns of expression of GRK2 in white blood cells can predict the outcome of the systolic cardiac function in a short time.

\section{RELATIONSHIP BETWEEN}

\section{ECHOCARDIOGRAPHIC AND FUNCTIONAL} PARAMETERS IN PATIENTS WITH HEART FAILURE UNDERGOING CARDIOPULMONARY EXERCISE TEST

Dominioni $\mathrm{I}^{1}$, Alessio $\mathrm{S}^{1}$, Russo $\mathrm{A}^{2}$, Cerasari $\mathrm{A}^{1}$, Sanesi $\mathrm{L}^{1}$, Filippucci $\mathrm{L}^{2}$, Vaudo $\mathrm{G}^{1}$, Pucci $\mathrm{G}^{1}$

1. Department of Medicine, University of Perugia, Unit of Internal Medicine, Terni University Hospital, Terni, Italy; 2. Unit of Rehabilitative and Preventive Cardiology, Grocco Center, Usl Umbria 1, Perugia, Italy

INTRODUCTION: HF patients typically show effort intolerance due to a reduction in peak exercise oxygen (peak VO2) consumption, which is related to inability to adapt systolic function to increased demand. Left ventricular ejection fraction (EF) is a surrogate marker of cardiac contractility and a powerful predictor of adverse prognosis in chronic heart failure (HF).

AIM: to explore the relationship between EF and other echocardiographic findings with peak VO2 in a population of $\mathrm{HF}$ individuals undergoing cardiopulmonary exercise testing (CPX).

METHODS: We evaluated 101 patients (61\% hypertensives, $74 \%$ with documented coronary artery disease) undergoing both resting echocardiography and symptomlimited CPX (COSMED Bike, personalized ramp protocol). The following parameters were evaluated: peak VO2, slope VE/VCO2, oxygen pulse, slope VO2/WR.

RESULTS: Mean age was $58 \pm 13$ years, $83 \%$ were males. Mean EF was $55 \pm 12 \% ; 20 \%$ of the patients showed $\mathrm{EF}<40 \%$. Mean test duration was $9.4 \pm 2.2 \mathrm{~min}$. Average peak VO2 was $21 \pm 6 \mathrm{~mL} / \mathrm{kg} / \mathrm{min}$. Peak VO2 showed a robust positive correlation with $\mathrm{EF}(\mathrm{R}=0.42$, $\mathrm{P}<0.001)$. Other independent predictors of peak VO2 were age, male sex, height and tricuspidal anular plane systolic excursion (TAPSE), this latter reflecting right ventricular dysfunction. When subjects were dichotomized according to predicted peak VO2 values, those with higherthan-predicted peak VO2 showed significantly lower VE/ $\mathrm{VCO} 2$ slope, and higher values of both oxygen pulse and VO2/WR slope.

CONCLUSIONS: EF and TAPSE are associated with peak VO2 in HF patients independently from age, sex and height. The evaluation of potentially relevant mechanisms affecting exercise capacity in HF patients could have therapeutic implications.

\section{THE ROLE OF INFERIOR VENA CAVA EVALUATION IN THE DIAGNOSIS OF ACUTE HEART FAILURE AMONG DYSPNOEIC PATIENTS}

Ilaria Fucile ${ }^{1}$, Alfonso Sforza ${ }^{2}$, Maria Viviana Carlino ${ }^{2}$, Mario Guarino ${ }^{2}$, Giovanni de Simone ${ }^{1}$, Nicola De Luca $^{1}$ and Costantino Mancusi ${ }^{1}$

Hypertension Research Center \&amp; Department of Advanced Biomedical Science, Federico II University Hospital, Naples, Italy; 2 Emergency Department, CTO Hospital, Naples, Italy

INTRODUCTION: Acute dyspnoea is one of the main reason for admission to the Emergency Department (ED). A rapid and accurate diagnosis can be lifesaving for these patients. Particularly, it is important to differentiate between dyspnoea of cardiac origin and dyspnoea of pulmonary origin.

AIM: to evaluate the real accuracy of the evaluation of diameter and collapsibility of IVC for the diagnosis of AHF among dyspnoeic patients.

METHODS: We analyzed 155 patients admitted for acute dyspnoea to the ED of "Maurizio Bufalini" hospital in Cesena (Italy) and "Antonio Cardarelli" hospital in Naples (Italy) from November 2014 to April 2017. All patients underwent ultrasound (lung-cardiac-inferior vena cava) examination with a hand-held device in addition to the traditional pathway. Patients were classified into AHF group or non-AHF group according to the current guidelines.

RESULTS: The final diagnosis was acute dyspnoea of cardiac origin in 64 patients and dyspnoea of non-cardiac origin in 91 patients. The diameter of IVC and the collapsibility of IVC showed low sensitivity $(70.3 \%$ and $76.6 \%$ respectively) and low specificity $(75.8 \%$ and $69.2 \%$ 
respectively) for the diagnosis of HF. AUC was 0.729 (0.647-0.811) for IVC collapse and 0.731(0.648-0.813) for IVC dilatation.

CONCLUSION: Our study demonstrated that the sonographic assessment of IVC diameter and/or collapsibility is not really accurate for differentiating acute dyspnoea due to AHF or other causes in the emergency setting.

\section{Epidemiology and Clinical Aspects}

\section{IMPORTANCE OF WORLD HYPERTENSION DAY IN THE SCREENING OF HYPERTENSION IN A SMALL TOWN IN LOMBARDY}

Boari GEM ${ }^{1}$, Bianco $\mathrm{G}^{1,2}$, Bonetti $\mathrm{S}^{1,2}$, Tagliani $\mathrm{L}^{1}$, Faustini $\mathrm{C}^{1,2}$, Braglia Orlandini $\mathrm{F}^{1,2}$, Turini $\mathrm{D}^{1}$, Guarinoni $\mathrm{V}^{1}$, Saottini $\mathrm{M}^{1}$, Rizzoni $\mathrm{D}^{1,2}$

\section{1- Department of General Medicine, Hospital} of Montichiari, ASST Spedali Civili Brescia, Montichiari (BS), Italy; 2-Department of Clinical and Experimental Science, University of Brescia, Brescia, Italy

INTRODUCTION: Cardiovascular disease is the leading cause of death in the developed world and hypertension represents one of the most relevant risk factor. World Hypertension Day is-since 2005-a worldwide initiative to raise people's awareness about hypertension-related disease and death. We have been performing this initiative in Scandolara Ravara (a small town nearby Cremona) since 2014.

METHODS: Random oxygen saturation, glycemia and total cholesterol as well as arterial blood pressure (three times) were measured in every person accessing to our stand.

RESULTS: A group of 202 subject were included (112 females and 89 males). Mean age was 68 years, mean weight $72.4 \mathrm{~kg}$ (females: $67.4 \mathrm{~kg}$.; men $78.7 \mathrm{~kg}$ ); mean BMI was 26.3 (females 25.9; men 26.8). Data are summarized in table 1 and 2. Among patients treated for any cause, mean number of pills was 3.3; among hypertensives, mean number of anti-hypertensive drugs was 1.8. Arterial blood pressure $(p<0.01)$ and heart rate $(p=0.002)$ were significantly higher during first measure compared to second and third check, confirming once more the importance of national and international societies indication on blood pressure measurement. During World Hypertension Day we identified 13 newly diagnosed hypertensives, 14 patients with high cholesterol level and one new diabetes.
Patients were addressed to their General Practitioner to undergo proper evaluation.

CONCLUSIONS: Our data confirm that adequate blood pressure control is still far from being achieved in a general population and demonstrate once more the importance of World Hypertension Day in screening and in raising awareness of the importance of risk factors in general population

Table 1 - Risk factors.
\begin{tabular}{|l|l|l|l|l|l|l|l|l|l|}
\hline & $\begin{array}{l}\text { Familiari } \\
\text { ty CV } \\
\text { disease }\end{array}$ & $\begin{array}{l}\text { CV } \\
\text { disease }\end{array}$ & $\begin{array}{l}\text { Hypertensi } \\
\text { on }\end{array}$ & $\begin{array}{l}\text { Diabete } \\
\text { s }\end{array}$ & $\begin{array}{l}\text { High } \\
\text { cholester } \\
\text { ol level }\end{array}$ & $\begin{array}{l}\text { Smok } \\
\text { e }\end{array}$ & $\begin{array}{l}\text { Low } \\
\text { Sodiu } \\
\text { m diet }\end{array}$ & $\begin{array}{l}\text { Exercis } \\
\text { e }\end{array}$ & $\begin{array}{l}\text { Alcoh } \\
\text { ol }\end{array}$ \\
\hline YES & 71 & 32 & 108 & 24 & 83 & 20 & 75 & 109 & 62 \\
\hline NO & 103 & 127 & 92 & 174 & 92 & 171 & 97 & 80 & 135 \\
\hline n.a. & 28 & 43 & 2 & 4 & 27 & 11 & 30 & 13 & 5 \\
\hline $\begin{array}{l}\text { YES } \\
\%\end{array}$ & $35 \%$ & $16 \%$ & $53 \%$ & $12 \%$ & $41 \%$ & $10 \%$ & $37 \%$ & $54 \%$ & $31 \%$ \\
\hline $\begin{array}{l}\text { NO } \\
\%\end{array}$ & $51 \%$ & $63 \%$ & $46 \%$ & $86 \%$ & $46 \%$ & $85 \%$ & $48 \%$ & $40 \%$ & $67 \%$ \\
\hline Na\% & $14 \%$ & $21 \%$ & $1 \%$ & $2 \%$ & $13 \%$ & $5 \%$ & $15 \%$ & $6 \%$ & $2 \%$ \\
\hline
\end{tabular}

Table 2 - Mean of measured parameters
\begin{tabular}{|l|l|l|l|}
\hline & Mis. 1 & Mis. 2 & Mis. 3 \\
\hline SBP (mmHg) & 149 & 145 & 144 \\
\hline DBP (mmHg) & 84 & 83 & 82 \\
\hline MBP (mmHg) & 105 & 103 & 101 \\
\hline FC (bpm) & 71 & 70 & 68 \\
\hline Oxygen Sat.\% & & $97,1 \%$ & \\
\hline Cholesterol (mg/dl) & 199,5 & \\
\hline Glycemia (mg/dl) & 104,5 \\
\hline
\end{tabular}

\section{PROGNOSTIC SIGNIFICANCE OF RADIOLOGIC "BRIXIA SCORE" IN PATIENTS AFFECTED BY SARS-COV-2 PNEUMONIA}

Boari GEM ${ }^{1}$, Malerba $\mathrm{P}^{1,2}$, Bonetti $\mathrm{S}^{1,2}$, Chiarini $\mathrm{G}^{1,2}$, Faustini $\mathrm{C}^{1,2}$, Braglia Orlandini $\mathrm{F}^{1,2}$, Bianco $\mathrm{G}^{1,2}$, Turini $\mathrm{D}^{1}$, Saottini $\mathrm{M}^{1}$, Guarinoni $\mathrm{V}^{1}$, Mascadri $\mathrm{C}^{1}$, Ferrari-Toninelli $\mathrm{G}^{1}$, Zanotti $\mathrm{E}^{1}$, Rizzoni $\mathrm{D}^{1,2}$

1-Department of General Medicine, Hospital of Montichiari, ASST Spedali Civili Brescia, Montichiari (BS), Italy; 2-Department of Clinical and Experimental Science, University of Brescia, Brescia, Italy

INTRODUCTION: Brixia score is a radiologic score proposed by Borghesi A and Maroldi R (Radiol Med 2020 May 1: 1-5) to assess severity of SARS-CoV-2 pneumonia. Aim of the present study was to assess le prognostic value of this score in a population of 78 patients admitted from 
29 February to 31 March 2020 to General Medicine ward of the Hospital of Montichiari (Bs).

METHODS: A group of 78 patients (54 males and 24 females, aged $69 \pm 13$ years) was included in the present study. In 75 patients, high-flow oxygen was required (Venturi mask), 6 patients needed orotracheal intubation and 10 were given continuous positive pressure oxygen therapy (CPAP). At the time of admission, a chest x-ray was performed, and the score was calculated. Till June 10th, 17 deaths had been observed. Survival curves were calculated according to Kaplan-Meier method. Comparison between groups was performed with Breslow and Mantel-Cox test.

RESULTS: A significant increase in mortality was observed in patients with a score higher than or equal to 8 (median value of the population- $\mathrm{p}=0.035$; Figure 1). Results were confirmed and even more consistent when using a more conservative cut-point $(10 ; \mathrm{p}=0.006)$. A significant correlation $(\mathrm{r}=-0.41, \mathrm{p}=0.006)$ between Brixia score and partial oxygen pressure and flow ratio $\left(\mathrm{pO}_{2} / \mathrm{FiO}_{2}\right)$ - a clinical hallmark of respiratory distress severity-was found.

CONCLUSIONS: Brixia score shows a relevant prognostic significance, allowing to detect patients with SARSCoV-2 at higher risk of poor outcome. A score higher or equal to 8 is related to a greater mortality in COVID-19 patients. The correlation becomes even stronger when choosing 10 as a cutoff.

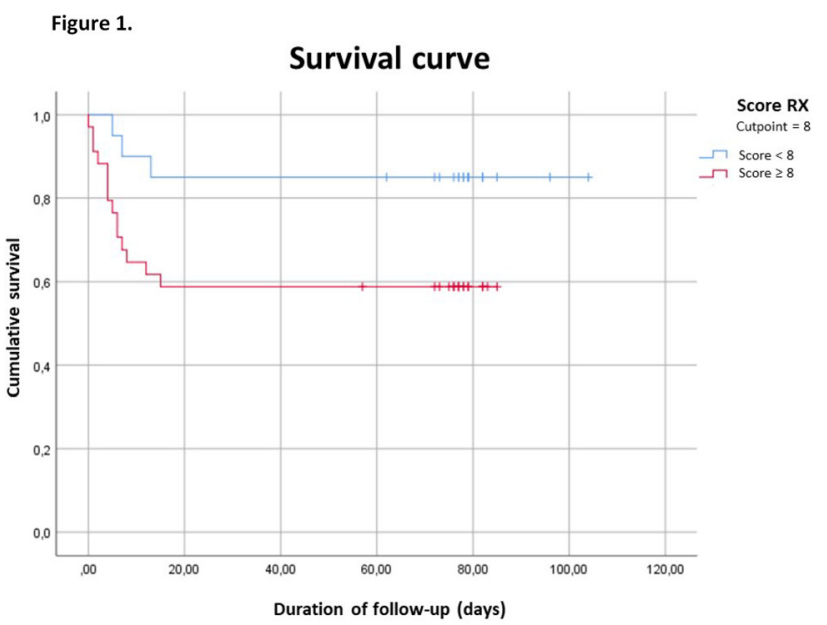

COGNITIVE SCREENING IN OLDER ADULTS WITH HYPERTENSION: A PILOT STUDY

Maria Flora D'Andria; Giulia Rivasi; Daniele Falzone; Giada Turrin; Virginia Tortu'; Evelina Giuliani; Antonella Giordano; Andrea Ungar

Hypertension Centre, Head Division of Geriatrics and Intensive Care Unit, University of Florence, Careggi Hospital, Florence, Italy

BACKGROUND: Hypertension is a recognized risk factor for cognitive impairment. Therefore, in older hypertensive adults cognitive screening tests should be included in the assessment of hypertension-related organ damage, as the 2018 ESH/ESC guidelines recommend. Numerous cognitive screening tests are available in the Literature, but their diagnostic accuracy in adults with hypertension has been scarcely investigated to date. The present study was carried out in a sample of older hypertensive adults without dementia and aimed at analyzing and comparing the diagnostic accuracy of the most widely used cognitive screening tests, including the MiniCog Test, the Montreal Cognitive Assessment (MoCA), the Mini Mental State Examination (MMSE) and the Clock Drawing Test (CDT). METHODS: The study was carried out in the Referral Centre for Hypertension Management in Older Adults, Careggi Hospital, Florence, Italy, between February 2017 and May 2019. Subjects aged 65 or older without a prior diagnosis of cognitive impairment were enrolled. Participants underwent a cognitive screening including the MMSE, the MoCa, the Mini-Cog and the CDT, followed by an extensive neuropsychological evaluation. Depressive symptoms and functional status were also assessed according to the Geriatric Depression Scale and the Basic and Instrumental Activities of Daily Life, respectively. Sensitivity and specificity of each screening test were assessed, using the ROC curves for the MMSE and the MoCA.

RESULTS: Among 94 participants, 35 (37.2\%) had a diagnosis of mild cognitive impairment or dementia at the neuropsychological evaluation. Among these, 7 (7.4\%) had a multiple domain cognitive impairment. According to the ROC curves, the MoCA and the MMSE achieved the best detection of cognitive impairment with a cut-off score of 24 (AUC 0.746) and 27.5 (AUC 0.689), respectively. The MoCA had the highest diagnostic accuracy, providing a $80 \%$ sensitivity and a $59 \%$ specificity. The MiniCog and the CDT had the lowest sensitivity $(48.6 \%$ and $51.4 \%$, respectively). A cognitive screening including both the Mini-Cog and the MMSE provided a higher sensitivity (74\%) and specificity (51\%) than the MMSE alone (sensitivity $69 \%$, specificity $52 \%$ ). 
CONCLUSIONS: Cognitive impairment has a high prevalence $(37.2 \%)$ in older hypertensive adults with no prior diagnosis of dementia. Among the available screening tests, the MoCA with a cut-off of 24 seems to have a good diagnostic accuracy in this population and could be included in the assessment of hypertension-related organ damage, to screen for cognitive impairment.

\section{PREVALENCE AND PREDICTORS OF HYPOTENSION IN NURSING HOME RESIDENTS UNDERGOING AMBULATORY BLOOD PRESSURE MONITORING}

Giulia Rivasi, Virginia Tortù, Maria Flora D'Andria, Giada Turrin, Evelina Giuliani, Martina Rafanelli, Enrico Mossello, Andrea Ungar

Referral Centre for Hypertension Management in Older Adults, Division of Geriatric and Intensive Care Medicine, Careggi Hospital and University of Florence, Italy

INTRODUCTION: In nursing home (NH) residents, hypotension may predispose to adverse events including falls, fractures and mortality. Yet, evidence on hypotension detected by ambulatory blood pressure monitoring (ABPM) is scarce in this setting.

AIM: to determine the prevalence and the clinical predictors of ambulatory hypotension in $\mathrm{NH}$ residents and to analyze its related ABPM profile.

METHODS: We retrospectively analysed data from a sample of $100 \mathrm{NH}$ residents undergoing a comprehensive geriatric assessment and ABPM. Ambulatory hypotension was defined as a systolic blood pressure (SBP) drop $\geq 20$ $\mathrm{mmHg}$ between two consecutive measurements to a minimum SBP $<100 \mathrm{mmHg}$. Participants with nocturnal hypotension were excluded from the analysis. Predictors of ambulatory hypotension were investigated using multivariate logistic regression adjusted for comorbidities, functional status and hypotensive medications.

RESULTS: Among 91 residents (mean age 83, 56\% female), 50 showed ambulatory hypotension. They had a higher prevalence of heart failure $(p=0.02)$ and atrial fibrillation $(\mathrm{p}=0.007)$ and more commonly received digoxin $(p=0.001)$ and benzodiazepines $(p=0.015)$. At ABPM they had lower $24 \mathrm{~h}$, daytime and night-time SBP $(\mathrm{p}<0.001)$ and more frequently showed a white coat effect $(\mathrm{p}<0.001)$ and a reverse-dipping profile $(\mathrm{p}=0.013)$. At multivariate analysis, benzodiazepines (OR 4.040), digoxin (OR 19.987) and creatinine $<1 \mathrm{mg} / \mathrm{dl}$ (OR 3.939) were independently associated with ambulatory hypotension.
CONCLUSIONS: Hypotension is common in NH residents undergoing ABPM and more frequently occurs in subjects with lower ambulatory SBP, a reverse dipping profile and a white coat effect. Benzodiazepines, digoxin and low creatinine are predictors of ambulatory hypotension in $\mathrm{NH}$ residents.

\section{COST-EFFECTIVENESS OF CARDIOVASCULAR RISK CLASSIFICATION BASED ON ANKLE- BRACHIAL INDEX TEST IN ASSOCIATION TO FRAMINGHAM RISK SCORE}

Maloberti $\mathrm{A}^{1,2}$, Cortesi $\mathrm{PA}^{3}$, Micale $\mathrm{M}^{3}$, Mazzaglia $\mathrm{G}^{3}$, Occhi $\mathrm{L}^{1,2}$, Palazzini $\mathrm{M}^{1,2}$, Peretti $\mathrm{A}^{1,2}$, Pansera $\mathrm{F}^{1,2}$, De Chiara $\mathrm{B}^{1,2}$, Vallerio $\mathrm{P}^{2}$, Mantovani $\mathrm{LG}^{3}$, Gianattasio $\mathrm{C}^{1,2}$

1. School of Medicine and Surgery, University of MilanoBicocca, Milan, Italy; 2. Cardiology 4, "A. De Gasperis" Department, Niguarda Ca Granda Hospital, Piazza Ospedale Maggiore 3, 20159, Milan, Italy; 3. Research Centre on Public Health (CESP), University of MilanBicocca, Monza, Italy

AIM: to assess the cost-effectiveness of ABI combine with FSR in primary prevention.

METHODS: A decisional analytical model was developed to simulate CV risk of asymptomatic female subjects, stratified in $3 \mathrm{CV}$ risk categories. The model compares the primary prevention intervention (treatment of subjects at intermediate and high risk) based on the classification with FRS alone or in association with ABI $<0.9$ (FRS-ABI). The cost-effectiveness model used 10 years time-horizon and the point of view of the Italian National Health System. Results were presented as incremental cost-effectiveness ratios (ICERs) per QALY gained. Cost and outcomes were discounted at $3 \%$.

RESULTS: FRS-ABI determined a reclassification to higher risks in $7 \%$ of the female population, with an increase in costs per patient ( $€ 3,373$ vs $€ 3,198)$ but also greater efficacy $(8,683$ vs 8,689 QALYs) over a 10-year time horizon, with an ICER of $€ 47.065$ per QALY gained. While using an ABI $<1.10$ cut-off we obtained an ICER of $€ 28.688$ per QALY gained over 10 years simulation. In the probabilistic sensitivity analysis, the FRS-ABI reported a $50 \%$ probability to be cost-effective with $\mathrm{ABI}<0.9$ cutoff and $80 \%$ probability with $\mathrm{ABI}<1.1$ at a willingness to pay threshold of $€ 50,000$ per QALY.

CONCLUSIONS: Our study showed that FRS and ABI combination is a potentially cost-effective CV risk classification approach in female population. Our results can 
help the healthcare decision makers in implementing better strategies for primary CV diseases prevention in Italy.

\section{RAISED BLOOD PRESSURE IN THE ITALIAN GENERAL ADULT POPULATION: PRELIMINARY RESULTS OF THE CUORE PROJECT SURVEY 2018-2019}

Chiara Donfrancesco, Anna Di Lonardo, Daniela Minutoli, Cinzia Lo Noce, Brigitta Buttari, Elisabetta Profumo, Paolo Bellisario ${ }^{\text {a }}$, Serena Vannucchi, Simona Giampaoli, Ferruccio Galletti ${ }^{\mathrm{b}}$, Pasquale Strazzullo ${ }^{\mathrm{b}}$, and Luigi Palmieri

${ }^{a}$ Istituto Superiore di Sanità, Rome, Italy; ${ }^{a}$ Italian Ministry of Health, Rome, Italy; ${ }^{b}$ Federico II University of Naples Medical School, Naples, Italy

INTRODUCTION: The WHO Global Action Plan for the Prevention of Non-Communicable Disease (WHO-NCD) recommends a $25 \%$ relative reduction in the prevalence of raised blood pressure (RBP) by 2025 .

AIM: In the perspective of achieving this target, prevalence of RBP and mean blood pressure were measured in the Italian general adult population in the framework of the 2018-2019 CUORE Project-Health Examination Survey.

METHODS: Within the health examination survey conducted in 2018-2019, representative random samples of resident population, aged 35-74 years, stratified by age and sex were examined in 10 Italian Regions from Northern, Central and Southern Italy. Blood pressure was measured at the right arm, in sitting position, by automated oscillometric device using standardized procedures and methods; the average of two measurements performed with $1 \mathrm{~min}$ interval was used for the analysis. RBP is defined as systolic and/or diastolic blood pressure equal or greater than $140 / 90 \mathrm{mmHg}$ or being under specific drug treatment. The survey is funded by the Italian Ministry of Health-CCM.

RESULTS: Preliminary analysis regarding 9 regions (930 men e 968 women) shows that prevalence of RBP is $43 \%$ (95\% Confidence Interval-CI 40-46\%) in men and 31\% (28-34\%) in women. In men and women prevalence of RBP is significantly higher in the Southern Regions. Mean value of systolic blood pressure is $131 \mathrm{mmHg}(127-135$ $\mathrm{mmHg}$ ) in men and $122 \mathrm{mmHg}(118-126 \mathrm{mmHg})$ in women. Mean value of diastolic blood pressure is 77 $\mathrm{mmHg}(76-78 \mathrm{mmHg}$ ) in men and $73 \mathrm{mmHg}(72-74$ $\mathrm{mmHg}$ ) in women.

CONCLUSIONS: These preliminary data underline that RBP is still high in the Italian adult population. Further analyses using the complete data will allow to make comparisons with similar previous surveys and to evaluate if Italy can achieve the WHO objective.

\section{ISOLATED SYSTSOLIC HYPERTENSION: CLINICAL CHARACTERISTICS AND HAEMODYNAMIC TIME-COURSE IN THE YOUNG}

\author{
F. Saladini, L. Mos, O. Vriz, A. Mazzer, C. Fania, \\ M Rattazzi, P. Palatini
}

INTRODUCTION: The prognosis of different hypertension subtypes, in the young is still debated, in particular in case of isolated systolic hypertension (ISH).

AIM: We examined baseline clinical characteristics and blood pressure (BP) time -course in young subjects with ISH comparing them with other hypertension subtypes.

METHODS: 1206 subjects (age 18-45 years) from the HARVEST study were involved: 81 normotensives (NT), 146 ISH, 281 isolated diastolic hypertensives (IDH) and 698 systolic-diastolic hypertensives (SDH). We examined baseline clinical and haemodynamic characteristics, BP and heart rate (HR) variations during 7 years of follow-up. RESULTS: ISH were younger $(25.6 \pm 6.6$ years, $\mathrm{p}<0.001)$ and more frequently males compared to the other subtypes, thinner compared to SDH $(24.6 \pm 2.6$ vs $\left.25.8 \pm 3.6 \mathrm{~kg} / \mathrm{m}^{2}, \mathrm{p}=0.028\right)$. HR was higher in ISH $(75.7 \pm 9.4 \mathrm{bpm})$ and SDH $(75.8 \pm 9.7 \mathrm{bpm})$ compared to the others $(\mathrm{p}<0.001)$. ISH were more active in sports $(55.5 \%, \mathrm{p}<0.001)$, while metabolic profile did not differ significantly among the groups. Systolic white-coat effect was higher among ISH $(17.6 \pm 12.4 \mathrm{mmHg})$ compared to the others $(\mathrm{p}<0.001)$. Cardiac output was higher among ISH $(6.3 \pm 1.2 \mathrm{ml} / \mathrm{min})$ compared to NT e IDH $(\mathrm{p}<0.001)$, while peripheral resistances were similar to those of NT and lower than ISH and SDH $(\mathrm{p}<0.001)$. During follow-up SBP decreased $(-7.6 \pm 14.4 \mathrm{mmHg})$ among ISH, while increased among NT and IDH $(\mathrm{p}<0.001)$. HR decreased in all categories but to a higher extent among ISH $(-3.8 \pm 10.9 \mathrm{bpm})$ and SDH $(-3.4 \pm 10.8 \mathrm{bpm}, \mathrm{p}=0.002$ versus NT). The percent of patients who started pharmacological treatment during follow-up was: $70.6 \%$ among SDH, $54.1 \%$ among IDH, $41.1 \%$ among ISH, and $39.5 \%$ among NT $(\mathrm{p}<0.001)$.

CONCLUSIONS: Young ISH showed higher white-coat effect, hyperkinetic state and lower peripheral resistances compared to the other hypertensive subtypes. Moreover, subjects with ISH showed a favourable time-course of BP and HR with a lower percent of subjects needing antihypertensive treatment, suggesting a lower risk profile. 


\section{DIAGNOSIS, MANAGEMENT AND TREATMENT OF HYPERTENSION EMERGENCY AND URGENCY ACCORDING TO DIFFERENT SPECIALTY OF PHYSICIANS: THE ITALIAN REAL WORLD}

Francesca Saladini ${ }^{1}$, Alessandro Maloberti ${ }^{2}$, Costantino Mancusi ${ }^{3}$, Fabio Bertacchini ${ }^{4}$, Francesco Spannella ${ }^{5}$, Alessandra Giavarini ${ }^{6}$, Martina Rosticci ${ }^{7}$, Rosa Maria Bruno ${ }^{8}$, Giacomo Pucci ${ }^{9}$, Martino Pengo ${ }^{10}$, Maria Lorenza Muiesan ${ }^{4}$

${ }^{1}$ Cardiologia, Ospedale di Cittadella, Padova, ITALY, ${ }^{2}$ Cardiology 4, ASST Niguarda Hospital, Milan, Milano, ITALY, ${ }^{3}$ Hypertension Research Center, Department of Advanced Biomedical Science, Federico II University of Naples, Napoli, ITALY, ${ }^{4}$ Internal Medicine, University of Brescia-ASST Spedali Civili di Brescia, Brescia, ITALY, ${ }^{5}$ Internal Medicine and Geriatrics, IRCCS-INRCA, Ancona, Ancona, ITALY, ${ }^{6}$ Cardiology Unit, Luigi Sacco Hospital, ASST Fatebenefratelli Sacco, Milano, Milano, ITALY, ${ }^{7}$ Medicine and Surgery Sciences Department, Alma Mater Studiorum University of Bologna, Bologna, ITALY, ${ }^{8}$ rtment of Clinical and Experimental Medicine, University of Pisa, Pisa, ITALY, ${ }^{9}$ Internal Medicine, Department of Medicine, University of Perugia, Perugia, ITALY, ${ }^{10}$ Department of Cardiovascular, Neural and Metabolic Sciences, IRCCS Istituto Auxologico Italiano, Milano, Milano, ITALY

INTRODUCTION: The management of hypertension emergency (HE) and urgency (HU) may vary across the Italian territory.

AIM: to investigate differences for management and treatment of HE and HU among emergency physician and doctors working in other departments.

METHODS: The young investigator research group of the Italian Society of Hypertension developed a 23-item questionnaire spread by e-mail invitation to the members of Italian Scientific societies involved in the field of emergency medicine and hypertension.

RESULTS: 665 questionnaires were collected (rate of response 55.42\%): $59.7 \%$ from emergency departments (EDs), 22\% from Emergency and Urgency Medicine wards, $8.7 \%$ from Cardiology Units, $5.7 \%$ from Internal Medicines and 3.9\% from Intensive care or Stroke units. Emergency physicians more frequently defined chest pain as possible presentation of $\mathrm{HE}$ than other physicians $(91.2 \%$ vs $85.8 \% \mathrm{p}<0.05)$, while tinnitus and conjunctival haemorrhages were less frequently considered as possible presenting symptoms ( $35 \%$ vs $42.9 \%, \mathrm{p}<0.05$ for tinnitus; $38.5 \%$ vs $45.9 \% \mathrm{p}=0.05$ for conjunctival haemorrhages). We did not observe any significant differences in most of laboratory and instrumental examinations, except for lung ultrasound that was most frequently performed by ED physicians compared the other specialists $(43.8 \%$ vs $32.1 \%$ $\mathrm{p}<0.005)$. They also investigated cocaine or amphetamine abuse more frequently than the others $(98.5 \%$ vs 95.9\% $\mathrm{p}<0.05)$. The therapeutic approach for blood pressure (BP) reduction showed some differences: a larger use of urapidil $(67.8 \%$ vs $54.9 \% \mathrm{p}=0.003)$ and captopril $(59.6 \%$ vs $39.2 \% \mathrm{p}=0.002)$ and lower of furosemide $(54.4 \%$ vs $66.8 \% \mathrm{p}<0.05)$ was reported by ED physicians compared to other groups. Hospitalization was more frequently required in the presence of acute organ damage $(45.3 \%$ vs $30 \% \mathrm{p}<0.05)$ by emergency physicians than other specialists, on the opposite side they suggested less frequently hypertension specialist evaluation (63\% vs $74 \%$, $\mathrm{p}<0.05)$ and ambulatory BP monitoring (65\% vs $79 \%$ $\mathrm{p}=0.001)$ or home self BP monitoring $(39.4 \%$ vs $56.1 \%$, $\mathrm{p}=0.003)$ compared to the other specialists, after patients discharge from ED.

CONCLUSIONS: Management and treatment of HE and HU may be different according to doctor's specialty. Educational initiatives, should be done to standardized treatment protocols for these clinical conditions.

\section{ADDING HOME AND/OR AMBULATORY BLOOD PRESSURE TO OFFICE BLOOD PRESSURE FOR CARDIOVASCULAR RISK PREDICTION}

Giuseppe Mancia ${ }^{1}$, Rita Facchetti ${ }^{2}$, Raffaella Dell'Oro $^{3}$,Cesare Cuspidi ${ }^{3}$, Giovanni Corrao ${ }^{2}$, Guido Grassi $^{3}$

${ }^{1}$ Policlinico di Monza and University Milano-Bicocca, Monza-Milano; ${ }^{2}$ National Centre for Healthcare Research and Pharmacoepidemiology, Department of Statistics and Quantitative Methods, University Milano-Bicocca, Monza-Milano; ${ }^{3}$ Clinica Medica, Department of Medicine and Surgery, University of Milano-Bicocca, MonzaMilano, Italy

INTRODUCTION: Home and $24 \mathrm{~h}(\mathrm{~h})$ blood pressure (BP)are believed to improve the prognostic value of office $\mathrm{BP}$ alone but the evidence has limitations such as that (1) these three BPs are characterized by multicollinearity and (2) the prognostic advantage of out-of-office BP is inferred by imperfectly accounting for the role of office BP via statistical adjustment procedures.

METHODS: We studied 1833 individuals belonging to the Pressioni Arteriose Monitorate e Loro Associazioni (PAMELA) population who had all three BP measurements and were followed for an average of 16.5 years. Prediction of cardiovascular (CV) and all-cause mortality was 
determined via the goodness of fit of individual data to the proportional hazard Cox model, the area underlying the receiving operator curves and the net reclassification improvement of mortality risk.

RESULTS: With each method, calculations were made for Office BP alone and after addition of home BP, $24 \mathrm{~h}$ BP or both, limited to their residual portion which was found to be unexplained by, and thus independent on, office BP. With all methods addition of residual out-of-office systolic(S) or diastolic (D) BP to office BP values significantly improved prediction of $\mathrm{CV}$ and, to a less consistent extent, all-cause mortality, although the improvement was always quantitatively modest. The improvement was consistent when home rather than $24 \mathrm{~h}$ BP was added to office BP and, compared to office plus home BP, no better prediction was found when addition extended to $24 \mathrm{~h} \mathrm{BP}$ as well.

CONCLUSIONS: Thus, addition of out-of-office to office $\mathrm{BP}$ measurements improves quantification of $\mathrm{CV}$ risk, even when data analysis avoids previous limitations. The improvement seems to be, quantitatively, limited, however.

\section{ADRENERGIC ACTIVATION IN TYPE 1 AND TYPE 2 DIABETES MELLITUS: META-ANALYSIS OF PUBLISHED STUDIES}

Seravalle $\mathrm{G}^{1}$, Dell'Oro $\mathrm{R}^{2}$, Quarti Trevano $\mathrm{F}^{2}$, Biffi $\mathrm{A}^{3}$, Corrao $\mathrm{G}^{3}$, Grassi $\mathrm{G}^{2}$, Mancia $\mathrm{G}^{2}$

${ }^{1}$ Cardiology Department, IRCCS S. Luca Hpt, Istituto Auxologico Italiano, Milano; ${ }^{2}$ Clinica Medica, Department of Medicine and Surgery, University of Milano-Bicocca, ${ }^{3}$ Department of Statistics and Quantitative Methods, Division of Biostatistics, Epidemiology and Public Health, University Milano-Bicocca, Milan, Italy

INTRODUCTION: Muscle sympathetic nerve traffic (MSNA) recordings have shown that sympathetic activation may characterize diabetes mellitus (DM). However, relevant questions related to the confounding role of clinical conditions frequently associated with DM, the sympathetic behaviour in type 1 and 2 DM as well as the relationships with metabolic variables remain undisclosed. METHODS: The present meta-analysis evaluated 11 microneurographic studies including 314 DM patients and healthy controls, the main variable of interest being MSNA. Assessment was extended to the relationships of MSNA with indirect adrenergic markers, hemodynamic, anthropometric and metabolic variables.

RESULTS: DM displayed MSNA values (means \pm SEM) significantly greater than controls (mean difference amounting to 8.1 , CI 95\% 1.21-15.08, $\mathrm{P}<0.05)$. This difference, which was detected even when confounders were excluded, was mainly ascribed to type $2 \mathrm{DM}$, since type 1 DM patients displayed MSNA values superimposable to those detected in healthy non-DM controls. In DM MSNA bursts corrected for heart rate was directly related to age $(\mathrm{r}=0.83, \beta=0.82, \mathrm{P}<0.04)$ and plasma insulin $(\mathrm{r}=1.00, \quad \beta=2.25, \quad \mathrm{P}<0.01)$ but not with plasma triglycerides, plasma glucose, clinic blood pressure and body mass index.

CONCLUSIONS: The DM related sympathetic activation is (1) detectable even when obesity, hypertension and metabolic syndrome is excluded, (2) restricted to type 2 DM, (3) unrelated to heart rate, anthropometric and hemodynamic values and (4) closely related to plasma insulin levels.

\section{ASSOCIATION BETWEEN THE ESC/ESH HEART RATE THRESHOLDS FOR CARDIOVASCULAR RISK AND NEUROADRENERGIC MARKERS}

Fosca Quarti-Trevano ${ }^{1}$, Gino Seravalle ${ }^{2}$, Raffaella Dell'Oro ${ }^{1}$, Rita Facchetti ${ }^{1}$, Giuseppe Mancia ${ }^{3}$, Guido Grassi $^{1}$

${ }^{1}$ Clinica Medica, Department of Medicine and Surgery, University of Milano-Bicocca; ${ }^{2}$ Cardiologia, Istituto Auxologico Italiano, IRCCS Osp. San Luca, Milano; ${ }^{3}$ University Milano-Bicocca and Policlinico di Monza, Monza-Milan, Italy

INTRODUCTION: The recent ESC/ESH hypertension guidelines identify resting heart rate (HR) values $>80$ beats/minute as predictors of cardiovascular risk, with the unproven assumption that this might reflect the presence of a sympathetic overdrive.

AIM: In the present study we tested this hypothesis throughout the use of direct and indirect sympathetic markers.

METHODS: In 193 untreated moderate essential hypertensives aged $50.4 \pm 0.6$ years (mean \pm SEM), we measured clinic and ambulatory blood pressure (BP) and corresponding HR, venous plasma norepinephrine (NE, HPLC) and muscle sympathetic nerve traffic (MSNA, microneurography). We then subdivided the study population in 2 groups according to $\mathrm{HR}<$ or $>80$ beats/ minute.

RESULTS: Eighty-four patients displayed resting HR > 80 beats/minute which was this cutoff value in the remaining 109 patients, the 2 groups showing superimposable age and gender distribution. Clinic and ambulatory BP were similar in the 2 groups while LVMI was significantly greater in the group with HR $>80$ beats/minute. MSNA values were also significantly greater in this latter 
group $(72.77 \pm 0.9$ vs vs $36.83 \pm 1.3$ bursts/minute, $\mathrm{P}<0.0001)$, this being the case also for NE $(293.0 \pm 8.7$ vs $254.1 \pm 8.9 \mathrm{pg} / \mathrm{ml}, \mathrm{P}<0.002)$. In the whole population there was a significant direct relationship between MSNA, NE, LVMI and HR values. Similar results were obtained when 24-hour HR values were analyzed.

CONCLUSIONS: Thus hypertensive patients displaying HR $>80$ beats/minute are characterized by a marked sympathetic overdrive, particularly when direct adrenergic markers are employed. This finding suggests that cardiac and peripheral sympathetic activation are involved in the increased cardiovascular risk detected in this group of patients.

\section{OFFICE AND OUT-OF-OFFICE BLOOD PRESSURE CHANGES OVER A QUARTER OF A CENTURY: FINDINGS FROM THE PAMELA STUDY}

Cesare Cuspidi ${ }^{\text {ab }}$, Rita Facchetti ${ }^{\text {a }}$, Raffaella, Dell'Oro ${ }^{a}$, Fosca Quarti-Trevano ${ }^{\mathrm{a}}$, Marijana Tadic ${ }^{\mathrm{c}}$, Giuseppe Mancia $^{\mathrm{d}}$ and Guido Grassi ${ }^{\mathrm{a}}$

${ }^{a}$ Department of Medicine and Surgery, University of Milano-Bicocca, Milano, Italy; ${ }^{b}$ Istituto Auxologico Italiano IRCCS, Milano, Italy; ${ }^{c}$ University Hospital "Dr. Dragisa Misovic_Dedinje” Department of Cardiology, Heroja Milana Tepica 1, 11000 Belgrade, Serbia, ${ }^{d}$ University of Milano-Bicocca and Policlinico di Monza, Italy

INTRODUCTION: Findings regarding long-terms variations in blood pressure (BP) taken in different setting (i.e. office, home and ambulatory BP) in the community are scanty. We sought to assess this issue in members of the general population enrolled in the Pressioni Monitorate E Loro Associazioni (PAMELA) study.

METHODS: The study included 562 participants who attended the second and third survey of the PAMELA study performed after 10 and 25 years from the initial evaluation. Data collection. included medical history, anthropometric parameters, office, home, ambulatory BP, and standard blood examinations

RESULTS: Office, home and 24-h systolic BP (SBP) over the 25-year interval between the first and third survey increased in a parallel way (i.e. $12 \%, 10 \%$ and $15.5 \%$ ). The increments in office, home and 24-h diastolic BP (DBP) were lower than the SBP ones (i.e. 3.3\%,5.6\% and 6.1\%). Thus, the combined changes in SBP and DBP from the first to the third data collection resulted in a marked increase in PP (i.e. 29\%, 19\% and 30\%). The prevalence of hypertension assessed at office visits and out-of-office either by self-BP measurements at home and ABPM increased approximately 3-4 times (3.1 office, 3.3 home, 3.9 ABPM, respectively). This trend was associated with adiposity indexes and worsening of the glucose profile.

CONCLUSIONS: This community-based longitudinal study suggests that the progressive and marked increase in hypertension with age, consistently documented with different BP measurement methods, represents an epochal challenge for the prevention of cardiovascular diseases, due to the rapid growth the elderly population worldwide

\section{HYPERTENSIVES MANAGEMENT IN COVID-19 POST EMERGENCY PHASE}

Erica Zaninelli ${ }^{1}$, Enzo Contursi ${ }^{2}$, Giuseppe Maso ${ }^{3}$, Irma Scarafino ${ }^{2}$, Giovambattista Desideri ${ }^{4}$, Claudio Ferri $^{4}$, Augusto Zaninelli ${ }^{5}$

${ }^{1}$ Corso Triennale per Medico di Medicina Generale, Polo di Bergamo; ${ }^{2}$ Medico di Medicina Generale, Bari, ${ }^{3}$ Medico di Medicina Generale, Venezia; ${ }^{4}$ Università degli Studi, L'Aquila; ${ }^{5}$ The System Academy, Firenze

The hypertensives People/Patient management, for almost all cases, oversees outpatient medicine, both primary and specialist care. The COVID-19 pandemic has produced a dramatic change in the clinical approach to patient care, hypertensives included. The post-emergency period needs mandatory relational changes for viral contagion prevention, even if continue medical assistance for risk factors control and prevention of cardio-cerebrovascular diseases is required. For this, teleconsulting, telemonitoring, digital health, patient's awareness, home equipment of tools for self-measurement of blood pressure values and other findings useful for remote management, are needful for a new doctor/patient relationship and an innovative kind of clinical governance. It's essential to strengthen the IT systems to create a network involving Patients/Caregivers, Specialist Doctors and General Practitioners to develop better control values and achieve clinical targets, particularly improving patient's adherence and doctor's clinical inertia.

\section{IN AN ITALIAN GENERAL POPULATION, CHRONIC CAFFEINE CONSUMPTION IS ASSOCIATED TO LOWER BASAL HEART RATE AND INDEPENDENTLY TO LOWER INCIDENCE OF CARDIOVASCULAR EVENTS}

Tikhonoff $\mathrm{V}^{1}$, Casiglia $\mathrm{E}^{2}$, Albertini $\mathrm{F}$, Toniazzo $\mathrm{S}^{1}$, Meraglia $\mathrm{F}^{1}$, Bindolo $\mathrm{MC}^{1}$, Ciaghi $\mathrm{G}^{1}$, Spinella $\mathrm{P}^{1}$ 
${ }^{1}$ Department of Medicine, University of Padova,Italy;

${ }^{2}$ Studium Patavinum, Department of Medicine, University of Padova, Italy; ${ }^{3}$ Azienda Ospedaliera-Università di Padova, Italy

AIM: we investigated the role of caffeine intake in the relationship between basal heart rate (HR) and incidence of cardiovascular events.

METHODS: In 1668 unselected subjects of Italian general population (751 men, mean age $59.6 \pm 17.1$ years) followed-up for $7.9 \pm 2.6$ years, we first performed multiple regression adjusted for confounders (age, sex, body mass index, diabetes, LDL-cholesterol and arterial hypertension) to identify the possible association between daily caffeine intake from all origin (coffee, tea, chocolate, cola beverage) the basal heart rate. Then, we applied mean of Cox analyses.

RESULTS: The lower the basal HR, the higher the daily caffeine consumption with an odds ratio of 0.996 (95\%CI 0.994-0.998, $\mathrm{p}=0.002$ ). In the four quartile of caffeine (i.e. $57 \pm 32,101 \pm 23,227 \pm 63$ e $344 \pm 80 \mathrm{mg} / \mathrm{die}$ ), basal HR was $70.1 \pm 10.8,70.0 \pm 10.5,68.4 \pm 10.5^{*}$ and $67.4 \pm 10.2 \mathrm{bpm}$, respectively (*p $<0.01$ vs. first quartile; $\mathrm{p}$ for trend 0.005). In longitudinal analysis, total fatal and non-fatal cardiovascular events were inversely associated to caffeine intake with a hazard ratio of 0.994 (95\% CI $0.009-0.998, p=0.009)$. In the four quartile of caffeine, incidence of total fatal and non-fatal cardiovascular events were $47.2 \%, 43.6 \% * *, 34.8 \% * *$ and $25.0 \%$, respectively $(* * \mathrm{p}<0.01$ vs. first quartile; $\mathrm{p}$ for trend 0.004$)$. However, strong caffeine users had the better cardiovascular outcome profile, independently of basal HR reduction (interaction term HR*affeine consumption non-significant in the Cox model). By mean of ROC curves, we identified the cut-off value of $>280 \mathrm{mg} /$ day of caffeine intake as the value associated with the lower cardiovascular risk (AUC 0.598, $\mathrm{p}<0.0001$; sensitivity 78.3 , specificity 40.5 ; Youden index 0.189 , 95\%CI $0.142-0.227$ ).

CONCLUSIONS: in general population, the stronger caffeine users have the lower basal HR and above 280 $\mathrm{mg} /$ day (corresponding to $3.5 \mathrm{cups} /$ day of Italian espresso coffee) they appears protected from cardiovascular events.

\section{URIC ACID AND ITS RELATIONSHIP WITH KIDNEY DISEASE MEASURES: THE URRAH STUDY}

Elisa Russo ${ }^{1}$, Giovanna Leoncini ${ }^{1}$, Francesca Viazzi ${ }^{1}$, Roberto Pontremoli ${ }^{1}$, Carlo M. Barbagallo ${ }^{2}$, Michele Bombelli $^{3}$, Edoardo Casiglia ${ }^{4}$, Arrigo F.G. Cicero ${ }^{5}$, Massimo Cirillo ${ }^{6}$, Pietro Cirillo ${ }^{7}$, Giovambattista Desideri $^{8}$, Claudio Ferri ${ }^{8}$, Ferruccio Galletti ${ }^{9}$, Loreto
Gesualdo $^{7}$, Guido Grassi ${ }^{3}$, Cristina Giannattasio ${ }^{3}$, Guido Iaccarino ${ }^{9}$, Francesca Mallamaci ${ }^{10}$, Alessandro Maloberti ${ }^{3}$, Stefano Masi ${ }^{11}$, Alberto Mazza ${ }^{12}$, Maria L. Muiesan $^{13}$, Pietro Nazzaro ${ }^{7}$, Paolo Palatini ${ }^{4}$, Gianfranco Parati ${ }^{3}$, Marcello Rattazzi ${ }^{4}$, Massimo Salvetti ${ }^{13}$, Valérie Tikhonoff ${ }^{4}$, Giuliano Tocci ${ }^{14}$, Andrea Ungar ${ }^{15}$, Giulia Rivasi ${ }^{15}$, Paolo Verdecchia ${ }^{16}$, Agostino Virdis ${ }^{11}$, Massimo Volpe ${ }^{14}, \&$ Claudio Borghi $^{5}$ on behalf of the Working Group on UricAcid and Cardiovascular Risk of the Italian Society of Hypertension

${ }^{1}$ Department of Internal Medicine, University of Genoa and Policlinico San Martino, Genoa; ${ }^{2}$ Biomedical Department of Internal Medicine and Specialistics, University of Palermo, Palermo; ${ }^{3}$ Clinica Medica, Department of Medicine and Surgery, University of Milano-Bicocca, Monza; ${ }^{4}$ Department of Medicine, University of Padua, Padua, Italy; ${ }^{5}$ Biomedical Department of Internal Medicine and Specialistics, University of Palermo, Palermo; ${ }^{6}$ Department of Medicine, University of Salerno, Salerno, Italy; ${ }^{7}$ Department of Medical Basic Sciences, Neurosciences and Sense Organs, University of Bari Medical School, Bari $^{8}$ Università degli Studi dell'Aquila, L'Aquila, Italy; ${ }^{9}$ Department of Public Health, "Federico II" University of Naples, Naples, Italy; ${ }^{10}$ CNR-IFC, Clinical Epidemiology of Renal Diseases and Hypertension, Reggio Cal Unit, Reggio Calabria; ${ }^{11}$ Department of Clinical and Experimental Medicine, University of Pisa, Pisa, Italy; 12 Department of Internal Medicine, Santa Maria della Misericordia General Hospital, AULSS 5 Polesana, Rovigo, Italy; ${ }^{13}$ Department of Clinical and Experimental Sciences, University of Brescia, Brescia; ${ }^{14}$ Department of Clinical and Molecular Medicine, University of Rome Sapienza, Sant'Andrea Hospital, Rome, Italy; ${ }^{15}$

Department of Geriatric and Intensive Care Medicine, Careggi Hospital and University of Florence, Florence, Italy; ${ }^{16}$ Hospital S. Maria della Misericordia, Perugia, Italy

INTRODUCTION: Hyperuricemia is commonly observed in patients with chronic kidney disease. However, a better understanding of the distribution of uric acid values across stages of GFR might be useful for risk stratification.

AIM: to study the relationships between serum uric acid and kidney disease measures in a large cohort of patients at cardiovascular risk from the URRAH study database.

METHODS: Clinical data of 26,971 patients were analyzed. Increased urinary albumin excretion was classified as micro or macroalbuminuria as appropriate. Factors associated with the presence of hyperuricemia defined on the basis of previously determined URRAH cut-offs for 
cardiovascular and total mortality were evaluated through multivariate analysis.

RESULTS: Mean age of study patients was $58 \pm 15$ years (51\% males, $62 \%$ with hypertension and $12 \%$ with diabetes), mean eGFR was $81 \mathrm{ml} / \mathrm{min}$ per $1.73 \mathrm{~m}^{2}$ with a prevalence of eGFR $<60$ and micro- or macro-albuminuria of 16, 15 and 4\%, respectively. Serum uric acid levels showed a trend towards higher values along with decreasing renal function. Both the prevalence of gout and the frequency of allopurinol use increased significantly with the reduction of eGFR and the increase in albuminuria. Factors independently related to the presence of hyperuricemia included male gender, eGFR strata, and signs of insulin resistance such as BMI and triglycerides.

CONCLUSIONS: The presence of low eGFR (i.e. $<60$ $\mathrm{ml}$ ) and or albuminuria entail a 50 to $70 \%$ greater likelihood of hyperuricemia. Chronic kidney disease measures are among the most relevant determinants of serum uric acid levels.

\section{CARDIOVASCULAR DISEASE AND HYPERTENSION: THE VERONA STUDY}

Giulio Rigon ${ }^{1}$, Martina Albrigi ${ }^{1}$, Giacomo Tebaldi ${ }^{1}$, Alice Giontella2, Cristiano Fava2, Pietro Minuz2

General Practitioner, Verona, Italy $^{1}$; Department of Medicine, University of Verona, Italy ${ }^{2}$

AIM: We performed an observational study aimed at defining prevalence of diabetes mellitus, heart failure, chronic kidney disease, atrial fibrillation previous myocardial infarction.

METHODS: We selected patients with diabetes mellitus, heart failure, chronic kidney disease, atrial fibrillation previous myocardial infarction among 43526 hypertensive patients living in the province of Verona (approximately one-third of the estimated total number). Information concerning antihypertensive treatment (the number of different drugs concomitantly prescribed over a period of 12 months among 13 drug categories), age, sex, blood pressure (categorical variable, cutoff $140 / 90 \mathrm{mmHg}$ ) was collected from their electronic records (database MySQL, Centro Studi FIMMG) by the 150 General Practitioners taking part in the study. Chi square test was used in the subgroup analysis. Logistic regression analysis was performed to define which variables were independent predictors of blood pressure control.

RESULTS: The total number of recorded hypertensives was 43526 (females 52.7\%), 19.3\% of them also had diabetes mellitus, $9.4 \%$ atrial fibrillation, chronic kidney disease, $2.9 \%$ heart failure and $2.2 \%$ a previous myocardial infarction. Data concerning blood pressure control in the previous 12 months was available for 26949 patients (females $51 . \%$ ). By logistic regression analysis, predictors of inadequate blood pressure control were, not being treated $(\mathrm{n}=2013)$, female sex, heart failure and chronic kidney disease $(\mathrm{P}<0.0001)$. When only patient that could ben defined as resistant to medication were considered (uncontrolled blood pressure control with 3 drugs or treated with 4 drugs or more, $n=6472$ ), the prevalence of diabetes mellitus $(32.8 \%)$, atrial fibrillation $(20.1 \%)$, heart failure $(9.1 \%)$, chronic kidney disease $(7.8 \%)$ myocardial infarction $(4.4 \%)$ was higher than in patients treated $(\mathrm{P}<0.0001)$.

CONCLUSIONS: In a large proportion of the population of hypertensives blood pressure control is not achieved. Overt cardiovascular diseases or diabetes mellitus in hypertensive patients are associated with poor blood pressure control, independently of the number of prescribed drugs, suggesting a vicious circle that could result in worsening of organ damage.

\section{EFFECTS OF CLINICALLY DIAGNOSED INSOMNIA AND RESTLESS LEGS SYNDROME ON NOCTURNAL ARTERIAL BLOOD PRESSURE: A SYSTEMATIC REVIEW AND META-ANALYSIS}

Giuseppe Maiolino $^{1 *} \mathrm{MD}, \mathrm{PhD}$, Valeria Bisogni ${ }^{2} * \mathrm{MD}$, Davide Soranna ${ }^{3} \mathrm{PhD}$, Martino F. Pengo ${ }^{3} \mathrm{MD}, \mathrm{PhD}$, Giacomo Pucci ${ }^{2}$ MD, PhD Cristiano Fava ${ }^{4}$ MD, Gian Luca Colussi ${ }^{5} \mathrm{MD}$, Carolina Lombardi ${ }^{3,6} \mathrm{MD}, \mathrm{PhD}$, Gianfranco Parati ${ }^{3,6}$ MD, Gian Paolo Rossi ${ }^{1}$ MD, Alessandro Silvani ${ }^{7} \mathrm{MD}, \mathrm{PhD}$, On behalf of the Sleep Disorders Working Group of the Italian Society of Hypertension

1 Hypertension Clinic, Department of Medicine - DIMED; University of Padova, Italy; 2 Unit of Internal Medicine, Terni University Hospital, Terni, Italy; 3 IRCCS Istituto Auxologico Italiano, Department of Cardiovascular, Neural and Metabolic Sciences, Milan, Italy; 4 Section of General Medicine and Hypertension, Dept of Medicine, University of Verona, Verona, Italy; 5 Internal Medicine, University of Udine, Udine, Italy; 6 Department of Medicine and Surgery, University of Milano-Bicocca, Milan, Italy; 7 Department of Biomedical and Neuromotor Sciences, University of Bologna, Bologna, Italy. * These authors have equally contributed to the abstract

INTRODUCTION: Nocturnal hypertension and disappearance of the physiological asleep blood pressure (BP) dipping are associated with an increased risk of 
cardiovascular events. Amid the common sleep disorders, chronic insomnia and restless legs syndrome (RLS) might affect the circadian blood pressure profile, but data are still controversial, partially owing to the diagnostic criteria heterogeneity.

AIM: The aim of this systematic review and meta-analysis was to evaluate the association of chronic insomnia and RLS with nocturnal hypertension and non-dipping BP pattern.

METHODS: The article search was performed in four different databases using predefined selection criteria, such as a clinical diagnosis of chronic insomnia or RLS, in order to increase the reliability of the results.

RESULTS: Of the 811 studies originally retrieved, four were finally selected. Three articles focused on RLS and showed increased night-time mean systolic BP $(5.6 \mathrm{mmHg}$, 95\% CI 0.1-11.0) compared to controls, while night-time mean diastolic BP did not differ significantly $(1.3 \mathrm{mmHg}$, 95\% CI -2.7 to 5.4). RLS patients exhibited an impaired systolic BP dipping compared with controls, as reported by the single study providing this data. Only one paper compared good sleepers and chronic insomnia patients, who featured a higher night-time mean systolic and diastolic $\mathrm{BP}$, as well as a decreased systolic BP dipping.

CONCLUSIONS: Although limited by the paucity of available studies, our systematic review and meta-analysis supports the evidence that chronic insomnia and RLS increases night-time systolic BP. Further clinical studies are warranted on the association between chronic insomnia, RLS, and nocturnal cardiovascular control.

\section{HIGH BLOOD PRESSURE IN ADOLESCENT: THE INFLUENCE OF LIFESTYLE AND ANTROPOMETRIC MEASURES ON ITS PREVALENCE ACCORIDNG TO THE EUROPEAN AND AMERICAN GUIDELINES}

G. Mule' (1), A. Sorce (1), K. Montalabano (1), V. Imbrogio (1), L. Peritore, C. (1), Gentile (1), E. Vicari (1), R. Dell'utri (1), G. Geraci (2), S. Cottone (1)

(1) ESH Hypertension Excellence Centre and Nephrology Unit-Department of Health Promotion, Mother and Child Care, Internal Medicine and Medical SpecialtiesUniversity of Palermo; (2) Dottorato di Ricerca in Medicina del Benessere, Nutrigenomica e Malattie degenerative Univ di Palermo

INTRODUCTION: The American Academy of Pediatrics (AAP) published in 2017 new guidelines for the screening and management of hypertension in children containing different indications and suggest different normal values compared to the European guidelines (ESH), leading to a reclassification of blood pressure values, the consequences of which are still little investigated.

METHODS: During the XV World Hypertension Day, blood pressure values of 1301 high school students aged between 13 and 18 were measured. Two questionnaires were administered, one relating to anamnestic data and anthropometric parameters and a second, aimed at investigating lifestyle. For the diagnosis of increased blood pressure, both ESH and AAP criteria were considered.

RESULTS: Applying the LG ESH the prevalence of high blood pressure values was 7.5\%, applying the AAP GL the prevalence stood at $14.1 \%$. These difference were not observed only in subjects with short stature, in students aged 15 and in male gender. With regard to the association with risk factors and lifestyle, the ORs are comparable between the two GLs with the exception of overweight, no physical activity and smoking. With regard to the screentime (time spent using a device such as smartphone, tablet or computer), a new emerging risk factor, there are significant differences only in the stratified analysis by gender and age.

CONCLUSIONS: Our study is still in progress, but from our results it seems that the use of nomograms based on the exclusion of overweight and fixed thresholds independent of gender or of an anthropometric parameter so changeable in this population such as height can represent a factor of confusion and overestimation, while a potential role of the screen time among the risk factors emerges.

\section{PREVALENCE OF ELECTROCARDIOGRAPHIC LEFT VENTRICULAR HYPERTROPHY AMONG HYPERTENSIVE URGENCIES AND EMERGENCIES}

Padoan Y, Villani C, Bisogni V, D’Abbondanza M, Cerasari A, Dominioni I, Santoni E, Vaudo G, Pucci G

Unit of Internal Medicine, Terni University HospitalDepartment of Medicine and Surgery, University of Perugia, Perugia, Italy

INTRODUCTION: hypertensive urgencies and emergencies are often found among people accessing the Emergency Departments (ED). Electrocardiography (ECG) has a fundamental role in distinguish hypertensive emergencies from urgencies, and it is also useful to diagnose left ventricular hypertrophy (LVH).

AIM: We evaluated LVH prevalence by ECG in hypertensive urgencies and emergencies. We also analyzed the ability of LVH-ECG to reclassify the cardiovascular (CV) risk. 
METHODS: We analyzed ECG traces of all patients admitted to the ED of the Terni University Hospital between February and April 2018. For LVH diagnosis, we applied the criteria proposed by the ESH/ESC guidelines: (1) Sokolow-Lyon $\left(\mathrm{S}_{\mathrm{v} 1}+\mathrm{R}_{\mathrm{v} 5 / \mathrm{v} 6} \geq 3.5 \mathrm{mV}\right)$; (2) Cornell $\left(\mathrm{S}_{\mathrm{v} 3}+\mathrm{R}_{\mathrm{avL}} \geq 2.8 \mathrm{mV}(\mathrm{m}), \geq 2.0 \mathrm{mV}(\mathrm{w})\right)$; (3) Cornell product $\left.\left(\mathrm{S}_{\mathrm{v} 3}+\mathrm{R}_{\mathrm{avL}}\right) \times \mathrm{qrs}(\mathrm{ms}) \geq 2440 \mathrm{~mm} . \mathrm{ms}\right)$; (4) $\mathrm{R}_{\mathrm{avL}} \geq 11 \mathrm{~mm}$; (5) Romhilt-Estes $\geq 5$, and the Perugia criterion $\left(\mathrm{S}_{\mathrm{v} 3}+\mathrm{R}_{\mathrm{avL}} \geq 2.4 \mathrm{mV}(\mathrm{m}), \geq 2.0 \mathrm{mV}\right.$ (w) or Romhilt-Estes $\geq 5$ or typical LV strain pattern). The risk reclassification was conducted according to ESC/ESH guideline.

RESULTS: among a total 148 patients with ECG readable for LVH $(1,3 \%$ of all the ED access, age $69 \pm 14$ years, male sex $41 \%$, BP 195/99 $\pm 28 / 19 \mathrm{mmHg}, 83 \%$ urgencies, $17 \%$ emergencies $)$, the $39 \%(n=57)$ had at least one LVHECG criterion. The different LVH prevalence between hypertensive emergencies (44\%) and urgencies (37\%) was not statistically significant $(\mathrm{p}=0,54)$. $\mathrm{LVH}-\mathrm{ECG}$ was more often found by applying the Perugia criterion $(n=47$, $32 \%)$ than with ESC/ESH criteria $(\mathrm{n}=42,28 \%)$. In $83 \%$ of subjects $(n=123)$ these two criteria were concordant. The application of the Perugia criterion reclassified a higher proportion of subjects $(n=36,24 \%)$ than $\mathrm{ESC} / \mathrm{ESH}$ criteria $(n=28,19 \%)$.

CONCLUSIONS: the prevalence of LVH-ECG among hypertensive urgencies and emergencies is high. LVHECG diagnosed at the ED in subjects with hypertensive urgencies/emergencies, especially if conducted by applying the Perugia criterion, is useful in identifying subjects at a very high $\mathrm{CV}$ risk level.

\section{CARDIOVASCULAR RISK AND OUTCOME IN PATIENTS WITH HYPERTENSIVE EMERGENCIES AND URGENCIES IN AN EMERGENCY DEPARTMENT: AN ITALIAN GREEK COLLABORATION}

Muiesan M.L. ${ }^{1}$, Salvetti M. ${ }^{1}$, Fragoulis C $^{2}$, Paini A. ${ }^{1}$, Aggiusti C. ${ }^{1}$, Bertacchini F. ${ }^{1}$ Stassaldi D. ${ }^{1}$, Tarozzi $\mathrm{L}^{1}$, Dimitriadis $\mathrm{K}^{2}$, Konstantinidis $\mathrm{D}^{2}$, Kasiakogias $\mathrm{A}^{2}$, Kalos $\mathrm{T}^{2}$, Andrikou $\mathrm{I}^{2}$, Siafi $\mathrm{E}^{2}$, Leontsinis $\mathrm{I}^{2}$, Iliakis $\mathrm{P}^{2}$, Tousoulis, Tsoufis $\mathrm{K}^{2}$

University of Brescia-ASST Spedali Civili Brescia, Italy ${ }^{1}$ \& National and Kapodfistrian university of Athens,

Hippocratio Hospital, Athens, Greece ${ }^{2}$

INTRODUCTION: at present, few data are available on the prognosis of hypertensive emergencies and urgencies admitted to Emergency Departments (ED).
AIM: to evaluate the incidence of total and cardiovascular events during follow-up in hypertensive patients admitted in 2 ED in Italy and Greece with hypertensive emergencies or urgencies.

METHODS: medical records of patients aged $>18 \mathrm{yrs,}$ admitted to the ED with blood pressure values $\geq 180$ $\mathrm{mmHg}$ (SBP) and/or $\geq 120 \mathrm{mmHg}$ (DBP) were collected and analysed (24\% of patients were classified as "hypertensive emergency" and $76 \%$ as "hypertensive urgency"). Data in 1218 patients (556 men and 662 women, mean age $70+13$ years) were analysed; the mean duration of follow-up after admission to the ED was $19.5+7$ months years.

RESULTS: During the follow-up cardiovascular events occurred in 148 patients (69 cardiac events, 43 cerebrovascular events). In 272 pts (22\%) a new episode of acute BP rise was recorded. A total of 87 deaths was recorded during follow-up (in 28 patients for cardiovascular causes). All cause and CV mortality were greater in patients with a previous hypertensive emergency (14.7 vs $4.7 \%$, Chi square $\mathrm{p}=0.0001$ and 5.8 vs $1.2 \%$ Chi square $\mathrm{p}<0.0001$ for all-cause and for CV mortality, respectively). The incidence of non-fatal cardiovascular events was 10.11 and 2.11 per 100 patient-years in patients with hypertensive emergency and urgency, respectively and similar results were obtained when we considered separately the occurrence of cerebrovascular events.

CONCLUSIONS: admission to the ED for hypertensive emergencies identifies hypertensive patients at increased risk for fatal and non-fatal cardiovascular events. Our results underline the need for an accurate follow-up in patients with hypertensive emergencies and urgencies.

\section{CAROTID ATHEROSCLEROSIS PREDICT BLOOD PRESSURE CONTROL IN HYPERTENSIVE PATIENTS: THE CAMPANIA SALUTE NETWORK}

Costantino Mancusi ${ }^{1}$, Raffaele Izzo1, Giovanni de Simone $^{1}$, Nicola De Luca ${ }^{1}$

Hypertension Center \& Department of Advanced Biomedical Sciences, Federico II University of Naples

INTRODUCTION: Routine carotid ultrasound is not recommended in the $2018 \mathrm{ESC} / \mathrm{ESH}$ arterial hypertension guidelines, as a tool to identify hypertension-mediated organ damage, unless clinically indicated. However, carotid plaque (CP), a landmark of carotid atherosclerosis (CA) is a strong correlate of increased arterial stiffness. Thus, CP might influence BP control overtime. Accordingly, assessed whether $\mathrm{CP}$ at the time of the first visit in 
our center could predict blood pressure (BP) control during follow-up (FU).

METHODS: From the Campania Salute Network registry, 6684 hypertensive patients had complete carotid ultrasound exam and were categorized by the presence of $\mathrm{CP}$ and baseline. Optimal BP control was define as average BP during FU $<140 / 90 \mathrm{mmHg}$.

RESULTS: At baseline, participants with CP were more likely to be males, older and exhibited higher systolic BP, lower diastolic BP, worse lipid profile and greater LV mass index and higher prevalence of LV hypertrophy (all $\mathrm{p}<0.0001)$ than patients without CP. The baseline differences in BP were also confirmed during follow-up (all $\mathrm{p}<0.0001)$. Optimal BP control was adjudicated in 59\% of patients. Presence of $\mathrm{CP}$ was significantly associated with the probability of uncontrolled BP during follow-up ( $p<0.0001$ ), for also independently of significant effect of older age, male sex, higher baseline BP values, classes of medication and presence of LV hypertrophy.

CONCLUSIONS: Presence of CP in treated hypertensive patients is associated with suboptimal BP control during FU, independently of older age, worse metabolic profile and presence of LV hypertrophy.

\section{PREVALENCE OF HYPOKALEMIA AND PRIMARY ALDOSTERONISM IN 5,100 PATIENTS REFERRED TO A TERTIARY HYPERTENSION UNIT}

Jacopo Burrello $^{1}$, Silvia Monticone ${ }^{1}$, Isabel Losano ${ }^{1}$, Giovanni Cavaglià ${ }^{1}$, Fabrizio Buffolo ${ }^{1}$, Martina Tetti ${ }^{1}$, Michele Covella ${ }^{1}$, Franco Rabbia ${ }^{1}$, Franco Veglio ${ }^{1}$, Barbara Pasini $^{2}$, Tracy Ann Williams ${ }^{1}$, Paolo Mulatero ${ }^{1}$

\section{${ }^{1}$ Division of Internal Medicine 4 and Hypertension Unit; Department of Medical Sciences, University of Torino, Torino, Italy; ${ }^{2}$ Medical Genetics Unit, Department of Medical Sciences, University of Turin, Turin, Italy}

INTRODUCTION: Historically, primary aldosteronism (PA) was considered a rare condition, always associated with hypokalemia. The widespread screening of patients with hypertension resulted into an increased prevalence of PA, with normokalemic hypertension being the most common phenotype. The Endocrine Society guideline recommend to screen for PA patients affected by hypertension and hypokalemia, but the prevalence of PA in patients with hypokalemia is unknown.

AIM: to define the prevalence of hypokalemia in referred patients with hypertension and the prevalence of PA in patients with hypokalemia and hypertension.
METHODS: This was a retrospective observational study. Between 2007 and 2018, 7,110 patients were referred to our tertiary hypertension unit; 5,100 had at least two visits to our center, concluded the diagnostic work-up for secondary hypertension and were included. To define hypokalemia $\left(\mathrm{K}^{+}<3.7 \mathrm{mmol} / \mathrm{L}\right)$ we considered the lowest recorded serum potassium concentration and the highest to define hyperkalemia $\left(\mathrm{K}^{+}>5.2 \mathrm{mmol} / \mathrm{L}\right)$.

RESULTS: The prevalence of hypokalemia was $15.8 \%$ $(804 / 5,100)$, whereas $76.9 \%$ were normokalemic, and $7.3 \%$ hyperkalemic. The prevalence of PA in patients with hypokalemia was $28.1 \%$ and increased with decreasing potassium concentrations up to $88.5 \%$ of patients with spontaneous hypokalemia and potassium concentrations below $2.5 \mathrm{mmol} / \mathrm{L}$. A multivariate regression analysis demonstrated the association of hypokalemia with the occurrence of cardiovascular events independent of PA diagnosis. An association of PA with the occurrence of cardiovascular events and target organ damage independent of hypokalemia was also demonstrated.

CONCLUSIONS: Our results confirm that PA is a frequent cause of secondary hypertension in patients with hypokalemia and the presence of hypertension and spontaneous hypokalemia are strong indications for PA diagnosis. Finally, we show that PA and hypokalemia are associated with an increased risk of cardiovascular events.

\section{ETHNIC DISPARITIES IN HYPERTENSION- MEDIATED ORGAN DAMAGE AND ITS USEFULNESS FOR THE CLINICAL MANAGEMENT OF SUBJECTS WITH GRADE I HYPERTENSION}

Denise Marcon ${ }^{1}$, Franca Morselli ${ }^{2}$, Ryan J McNally ${ }^{2}$, Bushra Farukh ${ }^{2}$, Lorenzo Nesti ${ }^{3}$, Philip J Chowienczyk $^{2}$, Luca Faconti ${ }^{2}$

${ }^{1}$ Dipartimento di Medicina, Sezione di Medicina Generale e Ipertensione, Università di Verona, Verona; ${ }^{2}$ King's College London, British Heart Foundation Centre, London; 3Laboratorio del Metabolismo, della Nutrizione e dell'Aterosclerosi, Dipartimento di Medicina Clinica e Sperimentale, Università di Pis

INTRODUCTION: Ethnic disparities in the prevalence of hypertension (HT) exist but data on hypertension-mediated organ damage (HMOD) are conflicting. Here we explored if ethnic differences in HMOD can be found in a dual ethnic cohort of subject with grade I HT and we tested if the evaluation of HMOD provides additional information for the clinical management of untreated patients compared to the estimation of 10-year cardiovascular risk (CVR). 
METHODS: In subjects with grade I HT and self-described ethnicity as "Black" or "White" HMOD was assess with albumin/creatinine ratio (ACR, urine spot), left ventricular mass index (LVMI, cardiac ultrasound) and carotid-femoral pulse wave velocity (cfPWV, Sphygmocor) alongside with brachial blood pressure (BP) measurements. In untreated subjects, 10-years CVR was estimated using QRISK3 calculator. RESULTS: 58 Black (26 female) and 61 White (17 female) subjects were recruited. White subjects were older compared to Black ones ( 49 years vs 43 years respectively) but there were no difference in their duration of hypertension $(\sim 4.5$ years $)$ and $\mathrm{BP}$ (mean \pm standard error) $146.3 \pm 0.8$ vs $146.8 \pm 0.9$ $\mathrm{mmHg}$ for systolic BP and $89.2 \pm 0.9$ vs $89.2 \pm 0.7$ for diastolic BP respectively). LVMI $(101.4 \pm 3.6$ vs $81.6 \pm 3.2) \mathrm{g} / \mathrm{m}^{2}$, ACR $(7.5 \pm 2.9$ vs $1.9 \pm 2.8) \mathrm{mmol} / \mathrm{mol}$ and cfPWV (10.6 \pm 0.4 vs $9.2 \pm 0.4) \mathrm{m} / \mathrm{s}$ were higher in Black subjects compared to White after adjustment for confounders (BP, age, gender, body mass index, creatinine, diabetes and dyslipidaemia); all $\mathrm{P}<0.05$. In untreated subjects $(\mathrm{n}=59), 14$ had evidence of HMOD including microalbuminuria, left ventricular hypertrophy and/or cfPWV $>10 \mathrm{~m} / \mathrm{s}$. Of these, 4 had CVR > $10 \%$.

CONCLUSIONS: For a similar level of BP, HMOD is more prevalent in Black compared to White subjects with grade I HT. Evaluation of HMOD is superior compared to CVR estimation in identifying subjects who may be started on medical treatment.

\section{Genetics and Pharmacogenomics}

\section{EXPLORATORY CASE-CONTROL STUDY ON ACE2 EXPRESSION IN CHILDREN WITH SHORT STATURE}

INTRODUCTION: Short stature is one of the most common presentations to paediatric endocrinologists. It is estimated that despite all the exams, in $50-90 \%$ of cases, children are labelled as having idiopathic short stature. Angiotensin converting enzyme (ACE)2 is the negative regulator of the renin angiotensin system, as it degrades Angiotensin (Ang)II and produces Ang1-7. It has been recently reported that genetic ACE2 deficiency is associated with reduced body weight as well as with impaired gestational weight gain and fetal growth restriction in pregnancy. It has been argued that ACE2 deficiency, which is usually associated with an increase of AngII, could be associated with prenatal as well as postnatal changes leading to reduced growth (such as uterine artery dysfunction and IGF-1 reduction, respectively).

AIM: Based on these premises, the aim of our study was to evaluate whether there was a difference of ACE2 expression in children with short stature as compared to age-matched controls.

METHODS: We designed an exploratory case-control study aiming at recruiting consecutively 40 children with short stature (cases) and 40 controls presenting at the Endocrinology Service, aged 2-13 years, excluding those with acute intercurrent diseases, diabetes, renal insufficiency, syndromes and/or on medications. After signing the informed consent to participating in the study, children underwent a medical visit and a fasting blood sampling. Peripheral blood mononuclear cells (PBMC) were isolated to extract mRNA for gene expression analyses. Sera were collected for protein measurements.

RESULTS: Children with short stature $(\mathrm{n}=29)$ presented with lower height and body weight as compared to controls $(n=29)$. Our preliminary data show that children with short stature exhibited a significant reduction of ACE2 gene expression, and a significant increase of ACE/ACE2 and AngII/Ang1-7 ratio. ACE/ACE2 ratio was inversely associated with weight, height, and BMI.

CONCLUSIONS: To our knowledge, this is the first study investigating ACE2 expression in a paediatric population. Consistent with the literature, our preliminary results show that ACE2 expression is significantly reduced in children with short stature. This study could represent the basis for further investigations aiming at establishing the presence of a causal relationship between ACE2 deficiency and growth reduction, with further diagnostic and therapeutic perspectives

\section{A NEW GENETIC RISK SCORE FOR BLOOD PRESSURE STRONGLY ASSOCIATES WITH THE INCIDENCE OF HYPERTENSION AND CARDIOVASCULAR ENDPOINTS IN TWO SWEDISH COHORTS}

\author{
A. Giontella1,2, M. Sjögren2, Luca Lotta3, John \\ Overton3, Aris Baras3, Regeneron Genetics Center, C. \\ Fava1,2, O. Melander2
}

\section{Department of Medicine, University of Verona, Verona, Italy; 2. Department of Clinical Sciences, Clinical Research Center, Lund University, Malmö, Sweden; 3. Regeneron Genetics Center, Tarrytown, NY, USA}

INTRODUCTION: The clinical value of the polygenetic component of blood pressure is commonly questioned. We constructed a new BP-GRS, including the most recently published variants genome wide significantly associated with either systolic or diastolic blood pressure (SBP, DBP), to investigate its association to the incidence of hypertension and cardiovascular endpoints in two urban-based 
cohorts: the Malmö Diet and Cancer $($ MDC, $\mathrm{n}=29295)$ and Malmö Preventive Project (MPP, $\mathrm{n}=$ 9367).

METHODS: The genotyping was performed with the Illumina GWAS chip (GSA array v1) and a weighted BP-GRS 858 based on 858 SNPs was calculated, normalized, and divided into deciles. Logistic and survival Cox regression models were used to test the associations of the new BP-GRS 858 with outcomes.

RESULTS: At baseline, we found a difference of $11.2 \mathrm{mmHg}$ (SBP) and $6 \mathrm{mmHg}(\mathrm{DBP})$ between top and bottom deciles of BP-GRS ${ }_{858}$. In MPP, the top vs bottom decile of $\mathrm{BP}^{-\mathrm{GRS}_{858}}$ was associated with doubled risk of incident hypertension (OR, 95\% CI 2.2, 1.7-2.8, p value: $2.0 \mathrm{E}^{-10}$ ). In MDC, when comparing top and bottom deciles of BP-GRS ${ }_{858}$, significant association was found between the age and sex adjusted BP-GRS $\mathrm{B}_{85}$ and the incidence of total cardiovascular events (HR, 95\% CI 1.5; $1.3-1.7$; p-value: $1.5 \mathrm{E}^{-12}$ ), stroke (HR, 95\% CI 1.6, $1.4-1.8 ; \mathrm{p}$ value: $2.1 \mathrm{E}^{-9}$ ), coronary artery disease (HR, $95 \% \mathrm{CI} 1.5,1.4-1.8 ; \mathrm{p}$ value: $1.3 \mathrm{E}^{-11}$ ), heart failure (HR, $95 \%$ CI $1.5,1.3-1.8, \mathrm{p}$ value: $2.5 \mathrm{E}^{-5}$ ), atrial fibrillation (HR, 95\% CI 1.2-1.1,1.4; p value:0.001) and total mortality (HR, 95\% CI 1.1, 1.0-1.2; p value:0.009).

CONCLUSIONS: Our findings confirm that adding novel genetic variants into a polygenic BP-GRS, despite very low effect sizes of individual variants, increases its predictive performance. BP-GRS ${ }_{858}$ contributes with clinically meaningful predictive information regarding future hypertension and CVD risk. Given that the exposure to high polygenetic risk starts at birth, we suggest that the BP-GRS ${ }_{858}$ might be useful to identify children or adolescents who would benefit from early hypertension screening and treatment.

\section{Resistant Hypertension}

\section{EFFECTIVENESS OF SACUBITRIL-VALSARTAN IN TREATMENT NOT ONLY OF HEART FAILURE, BUT ALSO OF RESISTANT HYPERTENSION: AN EMBLEMATIC CASE}

\author{
Milani M., Cartella I., Tognola C., Tavecchia G.,
} Grasso E., Sun J., Maloberti A., Giannattasio C. ${ }^{1}$

${ }^{1}$ ASST Grande Ospedale Metropolitano Niguarda, Milano

INTRODUCTION: We present the case of a 59-year-old woman, with hypertension diagnosed in 2014 and family history of cardiovascular disease. Since October 2018, the patient had been symptomatic for morning headache, dyspnoea at rest, orthopnea, palpitations, stabbing chest pain and occasional sense of constriction at neck. Severely elevated blood pressure was detected.
CASE REPORT: In December 2018 the patient was admitted to hospital for suspected coronary artery disease: the echocardiogram showed regional wall motion abnormalities (dyskinesia of the middle septum and hypokinesia of the lateral wall and of the middle segment of the infero-lateral wall), conditioning mild left ventricle systolic dysfunction (EF 45\%); signs of hypertensive cardiopathy were also observed (moderate mitro-aortic regurgitation and dilatation of the ascending aorta). However, coronary angiography did not show any significant coronary artery stenosis. As blood pressure measurements were still uncontrolled, despite optimized polypharmacological therapy, in May 2019 the patient was admitted to hospital for the work up of resistant hypertension. Renin/aldosterone ratio, urinary metanephrines and catecholamines were not indicative of secondary causes. We did not find stenosis of renal arteries or obstructive sleep apneas. Only hyperplasia of left adrenal gland was found (adenomas were absent), therefore an imaging follow up was started, which is currently negative. Cardiac MRI showed a dilated left ventricle, with increased thickness and severely depressed function (EF 30\%; 35\% according to the echocardiogram). Brain, retina and kidney damage was also found. Multiple attempts to modify medical therapy were made, trying to use many active principles at maximum dose, but they were only partially effective in blood pressure control. In July 2019, because of persisting severe systolic dysfunction after six months of optimized medical therapy (including bisoprolol, valsartan, spironolactone, chlortalidone, furosemide, doxazosin, nifedipine, clonidine), the patient was started on sacubitril-valsartan (titrated up to $97 / 103 \mathrm{mg}$ ).

CONCLUSIONS: Since September until today, there has been a progressive improvement in pressure control, so that the therapy was reduced to sacubitril-valsartan, amlodipine, spironolactone, bisoprolol, and in ventricular function (mild diffuse hypokinesis conditioning EF 48\%).

\section{Cerebrovascular Diseases}

\section{CENTRAL PULSE PRESSURE IS INVERSELY ASSOCIATED WITH PROXIMAL AORTIC REMODELING}

Anna Astarita $^{\mathrm{a}}$, Francesco Tosello ${ }^{\mathrm{a}}$, Andrea Guala ${ }^{\mathrm{b}}$, Fabrizio D'Ascenzo $^{c}$, Martina Bollati ${ }^{a}$, Dario Leone ${ }^{\mathrm{a}}$, Luca Sabia $^{\mathrm{a}}$, Giulia Bruno ${ }^{\mathrm{a}}$, Giulia Mingrone ${ }^{\mathrm{a}}$, Fabrizio Vallelonga ${ }^{\mathrm{a}}$, Eleonora Avenatti ${ }^{\mathrm{a}}$, Claudio Moretti ${ }^{\mathrm{c}}$, Franco Veglio ${ }^{\mathrm{a}}$, Luca Ridolfi ${ }^{\mathrm{d}}$, Alberto Milan ${ }^{\mathrm{a}}$

${ }^{a}$ Department of Medical Sciences, Division of Internal Medicine, Hypertension Unit, AO "Città della Salute e della Scienza” University Hospital, Turin, Italy; 
${ }^{b}$ Department of Cardiology, Vall d'Hebron Institute of Research, Barcelona, Spain; ${ }^{c}$ Department of Cardiology, AO "Città della Salute e della Scienza" University Hospital, Turin, Italy; ${ }^{d}$ DIATI, Politecnico of Turin, Italy

INTRODUCTION: Hypertension leads to aortic stiffening and dilatation but unexpected data from the Framingham Heart Study showed an inverse relationship between brachial pulse pressure and aortic diameter. Proximal aortic dilatation would be associated with lower pulse pressure, but would also predispose to a worse prognosis (cardiac events, heart failure).

AIM: to evaluate the relationship between invasivelymeasured central blood pressure and proximal aortic diameter adjusted for age, gender and body height ( $\mathrm{Z}$ score).

METHODS: In 71 consecutive patients referred to invasive hemodynamic study, proximal aortic remodeling was evaluated in terms of Z-score, comparing diameters measured at the Sinus of Valsalva to the diameter expected according to patients' age, gender and body height. Pressures were recorded directly in the proximal aorta by means of a catheter before coronary assessment.

RESULTS: In this cohort, aged $67 \pm 10$ years $(77.5 \%$ men) mean invasive aortic systolic and diastolic blood pressures were $146 \pm 23$ and $78 \pm 13 \mathrm{mmHg}$, respectively, giving a central pulse pressure (cPP) of $68 \pm 21 \mathrm{mmHg}$. Proximal aortic diameter was $34.9 \pm 19.4 \mathrm{~mm}$, while $\mathrm{Z}$-score was $-0.3 \pm 1.7$. In bivariate analysis, invasive cPP was inversely related to $\mathrm{Z}$-score $(\mathrm{R}=-0.271, \mathrm{p}=0.022)$ and positively related to age and mean blood pressure. Subjects with higher cPP showed a significantly lower Z-score ( -0.789 vs. 0.155 , $\mathrm{p}=0.001$ ). In a stepwise multiple regression analysis $\mathrm{Z}$-score at the sinuses of Valsalva was independently and inversely related to invasive aortic pulse pressure $(\beta=-0.241, p=0.011)$.

CONCLUSIONS: Aortic root Z-score is inversely associated with invasively-measured central pulse pressure in a cohort of subjects undergoing invasive coronary assessment. Remodeling at the sinuses of Valsalva may be a compensatory mechanism to limit pulse pressure.

\section{NEUROLOGICAL HYPERTENSIVE EMERGENCIES: CORRELATION OF BLOOD PRESSURE VALUES WITH IN-HOSPITAL MORTALITY AND DISCHARGE DISABILITY}

Valentina Giani ${ }^{1}$, Alessandro Maloberti ${ }^{1,2}$, Marco

Biolcati ${ }^{1}$, Sofia Bianchi ${ }^{1}$, Gloria Magni ${ }^{1}$, Valentina

Albertini ${ }^{1}$, Matilde Conti ${ }^{1}$, Camilla Curci ${ }^{1}$, Lorenzo
Porta $^{1}$, Annalisa rigamonti ${ }^{1}$, Andrea Trucchi ${ }^{1}$, Marta Battistini ${ }^{1}$, Marta Bergamaschi ${ }^{1}$, Tommaso Valobra ${ }^{1}$, Nicolò Capsoni ${ }^{1}$, Giulio Cassano ${ }^{1}$, Gheda Silvia ${ }^{1}$, Chiara Ceresa ${ }^{3}$, Elio Clemente Agostoni ${ }^{3}$, Andrea Beretta $^{4}$, Andrea Bellone ${ }^{4}$ and Giannattasio Cristina ${ }^{1,2}$

1-School of Medicine and Surgery, Milan-Bicocca University, Milan, Italy; 2 - Cardiology 4, ASST Niguarda Ca Granda Hospital, Milan, Italy; 3- Neurology, ASST Niguarda Ca Granda Hospital, Milan, Italy; 4- Emergency Department, ASST Niguarda Ca Granda Hospital, Milan, Italy

INTRODUCTION: definitive data on acute management of Blood Pressure (BP) in neurological Hypertensive Emergencies (HE) are still lacking.

AIM: to evaluate BP values and management as a determinant of in-hospital mortality and early complications in stroke patients.

METHODS: we collected data of 267 patients, who presented with ischemic stroke and $\mathrm{BP} \geq 180 / 120 \mathrm{mmHg}$ at the Emergency Department of Niguarda Hospital from 2015 to 2017. In-hospital mortality, hospitalization length and discharge disability (evaluated with modified Rankin score-mRs) were considered as outcomes.

RESULTS: Mean age was $75.7 \pm 11.7$ years with SBP values of $194.9 \pm 14.9 \mathrm{mmHg}$ at admission. $34.8 \%$ of the patients received anti-hypertensive treatment with those achieving a higher SBP reduction in comparison with the untreated one $(\triangle \mathrm{SBP} \quad 37.8 \pm 26.8 \mathrm{mmHg} \quad$ vs $30.7 \pm 20.8 \mathrm{mmHg} \mathrm{p}=0.034)$. At the multivariate analysis in the overall population, no SBP values are related to all causes in-hospital mortality. Instead, higher admission SBP relates to high discharge disability and hospitalization length. Furthermore, BP values at admission in Emergency Department appear as disability determinants in patients who did not receive systemic thrombolysis and in patients who did not receive antihypertensive drugs. In these two subgroups, higher SBP values at department entrance determine a higher in-hospital mortality.

CONCLUSIONS: In overall population, no BP values are related to all causes in-hospital mortality while higher admission BP relates to high disability and hospitalization length. 
Hormonal Mechanisms and Endocrine Hypertension

\section{CASE OF PRIMARY ALDOSTERONISM WITH DISCORDANT HORMONAL AND COMPUTED TOMOGRAPHIC FINDINGS}

\author{
Grasselli Chiara, Muoio Angela and Ghirarduzzi
} Angelo

C.S. Second Cardiovascular Medicine, Hypertension Unit; A.U.S.L.-I.R.C.C.S. Santa Maria Nuova Hospital of Reggio Emilia

CASE REPORT. A 36-year-old male, non-smoker, with hypercholesterolaemia, was at our Center for the recent onset of 'non-dipper' grade I hypertension at ABPM. Unfamiliarity or drugs, licorice or substances of abuse. After beginning of barnidipine $10 \mathrm{mg} /$ day controlled blood pressure values. Minimum potassium value $3.3 \mathrm{mmol} / \mathrm{L}$. At screening aldosterone $319 \mathrm{pmol} / \mathrm{L}$ and renin $1.6 \mathrm{mIU} / \mathrm{L}$ with elevated ARR. Cortisoluria and urinary metanephrines were normal. After saline load aldosteronemia $227 \mathrm{pmol} / \mathrm{L}$ with confirmation of primary hyperaldosteronism. On CT scan 11x11 mm solid nodule in the body of the left adrenal gland. At adrenal venous sampling, prevalent right aldosterone secretion (Table 1). Asymptomatic patient. No evidence of hypertensive heart disease. Subsequent videolaparoscopic right adrenalectomy with histological confirmation of the presence of adrenal cortical adenoma. Regular post-operative course with normalized blood pressure values. In Table 2 the clinical and laboratory characteristics of the patient before and after the adrenalectomy. The patient was initiated to AVS as young, with a history of short-lived hypertension and with biochemical evidence of primary hyperaldosteronism. In fact, it is known that in these cases the response to surgical therapy is usually complete; in addition, up to $48 \%$ of patients with primary hyperaldosteronism have normal adrenals on CT scan and over $32 \%$ of these have significant lateralization of aldosterone production, a form potentially correctable with surgery. The patient was therefore sent to surgery despite the discrepancy between AVS and imaging. The AVS is currently the diagnostic gold standard, although it is complicated to perform it and subsequently interpret the data, so the evaluation must be carried out at a dedicated center.

CONCLUSIONS: In our case, as a result of further documentation of AVS superiority, complete clinical and biochemical healing was obtained six months after the surrectomy. No genetic abnormalities have been identified.
CYP11B2 DNA METHYLATION PATTERN IN ALDOSTERONE-PRODUCING ADENOMAS AND IN CONCURRENT ALDOSTERONE-PRODUCING CELL CLUSTERS

\author{
F. Fallo ${ }^{1}$, L. Morandi ${ }^{2}$, B. Rubin ${ }^{1}$, C. Pilon ${ }^{1}$, \\ V. Maffeis ${ }^{3}$, S. Asioli ${ }^{2}$, V. Vicennati ${ }^{4}$, A. De Leo ${ }^{5}$, \\ F. Ambrosi ${ }^{6}$, D. Santini ${ }^{5}$, U. Pagotto ${ }^{4}$, A. Fassina ${ }^{3}$, \\ G. Di Dalmazi ${ }^{4}$
}

${ }^{1}$ Department of Medicine, Clinica Medica 3, University of Padova, Italy, ${ }^{2}$ Department of Biomedical and Neuromotor Sciences, Section of Anatomic Pathology, Bellaria Hospital, Bologna, Italy, ${ }^{3}$ Department of Medicine, Cytopathology Unit, University of Padova, Italy, ${ }^{4}$ Department of Medical and Surgical Sciences, Endocrinology Unit, University of Bologna, Italy, ${ }^{5}$ Pathology Unit, S. Orsola Policlinic, Bologna, Italy, ${ }^{6}$ Pathology Unit, Maggiore Hospital, Bologna, Italy

INTRODUCTION: Epigenetic mechanisms may regulate CYP11B2 gene function in primary aldosteronism. It has been hypothesized that aldosterone-producing cell clusters (APCCs) may become a source of autonomous aldosterone production when evolving into aldosterone-producing adenoma (APA).

AIM: to determine whether CYP11B2 DNA is differentially methylated in APAs and in their concurrent APCCs. METHOD: Eleven formalin-fixed paraffin-embedded adrenal tissues from patients with APA were studied. Immunohistochemical staining was performed using antiCYP11B1 and anti-CYP11B2 monoclonal antibodies. Staining was quantified by McCarty's H-score. APAs and satellite APCCs identified by immunohistochemistry were microdissected using manual core drilling, and genomic DNA was extracted for CYP11B2 methylation analysis. CYP11B2 DNA methylation level was measured by quantitative Bisulfite-NGS. Bio-informatic analysis was performed in a GalaxyProject environment and processed by BSPAT. The equation $2^{\wedge}$-ddCt was used to calculate the fold changes in gene expression between the different adrenal cell structures. Somatic DNA mutations in aldosterone-driver genes KCNJ5, ATP1A1, ATP2B3 and CACNA1D were analyzed by Sanger sequencing.

RESULTS: 8/11 APA specimens showed at least one concurrent APCC and 6/8 were available for molecular study. A wide range of CYP11B2 and CYP11B1 immunohistochemical expression was detected in APAs, while positive CYP11B2 and negative CYP11B1 staining was uniformly found in APCCs. The H-score for CYP11B2 expression was not different in all APCCs compared to APAs $(\mathrm{P}=0.078)$. CYP11B2 DNA methylation levels were lower in APAs than in concurrent APCCs 
$(0.51 \pm 0.25$ vs $0.82 \pm 0.16$ as mean $\pm \mathrm{SD}, \mathrm{P}<0.05)$. Five KCNJ5 and one ATP2B3 mutations were found overall in 11 APAs, and four KCNJ5 and one ATP2B3 mutations among the 6 APAs with concurrent APCCs. No somatic mutations were found in APCCs. CYP11B2 DNA methylation rate was not different in APAs with and without mutations (P ns).

CONCLUSIONS: CYP11B2 DNA methylation levels lower in APAs than in concurrent APCCs may sustain the hypothesis of APCC switching to autonomous aldosterone production via a CYP11B2 demethylation process.

\section{PARACARDIAC PARAGANGLIOMA OF THE AORTOPULMONARY WINDOW PRESENTING WITH SYNCOPE: A CASE REPORT}

\author{
Alessio Balletti ${ }^{1}$, Nicola Riccardo Pugliese ${ }^{1,}$ Katia \\ Raimo $^{1,}$ Stefano Taddei ${ }^{1,}$ Alessandra Violet Bacca ${ }^{1}$ \\ ${ }^{1}$ Department of Internal Medicine, University of Pisa, Pisa, \\ Italy
}

INTRODUCTION: Paragangliomas are rare extra-adrenal tumors originating from the neural crest-derived chromaffin cells of the sympathetic or parasympathetic ganglia. Their clinical presentation is variable, depending mainly on tumor location and secretion pattern. Cardiac paragangliomas have relevant mortality due to their localization.

CASE REPORT: A 72-year-old man presented to our emergency department for head and thoracic trauma following a witnessed transient loss of consciousness. The ATLS protocol, including a full-body CT scan, was executed and multiple rib fractures and a vascularized mass adjacent to the ascending aorta were found. After partial embolization of arterial branches supplying the mass, the patient was referred to our Internal Medicine Department for further evaluations. Syncope work-up (physical examination, blood tests, ECG, Holter monitoring, echocardiogram, carotid sinus massage) was negative except for the presence of orthostatic hypotension (clinostatic BP 134/82 mmHg HR 74 bpm, orthostatic BP time 0' 112/71 mmHg HR $77 \mathrm{bpm}$ ). An MRI revealed a 78x48x63mm vascularized, defined and solid intrapericardial tumor located within the aortopulmonary window, without bleeding signs. Morphological features suggested paraganglioma. Urinary normetanephrines and chromogranin A serum levels were elevated (normetanephrines $697 \mu \mathrm{g} / 24 \mathrm{~h}$ n.v. $<600$, metanephrines $91 \mu \mathrm{g} / 24$ h n.v. $<350, \mathrm{CgA}$ $563 \mathrm{ng} / \mathrm{ml}$ n.v. $<100)$. The 68 Ga-DOTATOC PET/CT confirmed the involvement of chromaffin tissue. The patient was submitted to alpha-beta blockade therapy (carvedilol 12,5 $\mathrm{mg}$ bid), showing a normotensive and dipper pattern on ambulatory blood pressure monitoring. Finally, he was discharged and submitted to surgery. Genetic analysis of the known susceptibility genes was performed (RET, VHL, SDHA, SDHAF2, SDHB, SDHC) and a wild type pattern was found.

CONCLUSIONS: This case confirms the challenging paragangliomas diagnosis due to their clinical variability and often intermittent secretive pattern, making them asymptomatic or paucisymptomatic for years.

\section{EVALUATION OF INTRARENAL STIFFNESS IN PATIENTS WITH PRIMARY HYPERALDOSTERONISM}

L. Petramala ${ }^{1}$, M. Mezzadri ${ }^{1}$, F. Circosta ${ }^{1}$, A. Concistrè ${ }^{1}$, M. Soldini ${ }^{1}$, G. Iannucci ${ }^{2}$, A. Gigante ${ }^{1}$, R. Cianci ${ }^{1}$, C. Letizia ${ }^{1}$

${ }^{1}$ Department of Translational and Precision Medicine, "Sapienza" University of Rome, Rome, Italy ${ }^{2}$ Department of Clinical, Internal Medicine, Anesthesiology and Cardiovascular Sciences, Sapienza" University of Rome, Rome, Italy

INTRODUCTION: Beyond its important role in increasing blood pressure values, aldosterone acts significantly on development of cardiovascular, metabolic, renal damage in patients affected by Primary Aldosteronism (PA).

AIM: to evaluate arterial subclinical markers of early renal damage obtained by intrarenal Doppler ultrasonography in patients affected by Essential Arterial hypertension (EH) and PA, without overt organ damage.

METHODS: We consecutively enrolled 73 hypertensive subjects [30 with EH (mean age $49.5 \pm 18.7$ years) and 43 with PA (mean age $57.1 \pm 11.6$ years)]. PA group included 19 with idiopathic aldosteronism (IHA) and 23 with aldosterone-secreting adrenal adenoma (APA).

RESULTS: PA patients showed higher levels of urinary albumin excretion $(38.9 \pm 16.7 \mathrm{mg} / 24 \mathrm{~h})$ and lower concentration of plasmatic creatinine $(0.80 \pm 0.18 \mathrm{mg} / 24 \mathrm{~h})$ than EH (respectively, $9.2 \pm 4.5 \mathrm{mg} / 24 \mathrm{~h} ; 0.98 \pm 0.2 \mathrm{mg} /$ $24 \mathrm{~h} ; \mathrm{p}<0.001)$. Using intrarenal Doppler ultrasonography study, compared to EH group, PA showed increased values of right and left Parietal Thickness (respectively, $18.1 \pm 3.5 \mathrm{~mm}$ vs $13.6 \pm 1.8 \mathrm{~mm} ; 17.98 \pm 3,4 \mathrm{~mm}$ vs $14.55 \pm 1.7 ; \mathrm{p}<0.001)$, increased values of right and left Atrophy Index (AI) (respectively, $1.49 \pm 0.13$ vs $0.65 \pm 0.07 ; 1.46 \pm 0.1$ vs $0.662 \pm 0.070 ; \mathrm{p}<0.001$ ) increased values of right and left Pulsatility Index (PI) (respectively, $\quad 1.28 \pm 0.28 \quad$ vs $\quad 1.14 \pm 0.3 \quad \mathrm{~s}-\mathrm{d} / \mathrm{v}$; $1.27 \pm 0.28$ vs $1.12 \pm 0.24 \mathrm{~s}-\mathrm{d} / \mathrm{v} ; \mathrm{p}<0.03)$ and higher percentage of patients with altered right and left Resistance 
Index $(>0.7 \mathrm{~cm} / \mathrm{s})$ (respectively, $12 \%$ vs $6 \% ; 10 \%$ vs $6 \%$; $\mathrm{p}<0.001)$. Finally, in overall population, plasmatic aldosterone levels were positively correlated to AI $(\mathrm{r}=0.55 ; \mathrm{p}<0.05)$ and PI $(\mathrm{r}=0.35 ; \mathrm{p}<0.05)$ (Figure 2$)$. CONCLUSIONS: Beyond higher 24-hours urinary albumin excretion, this study showed greater subclinical renal damage in PA patients, characterized by altered arterial Stiffness parameters, assessed through the intrarenal Doppler ultrasonography study; moreover, these alterations were significantly correlated to plasmatic aldosterone levels.

Figure 2.

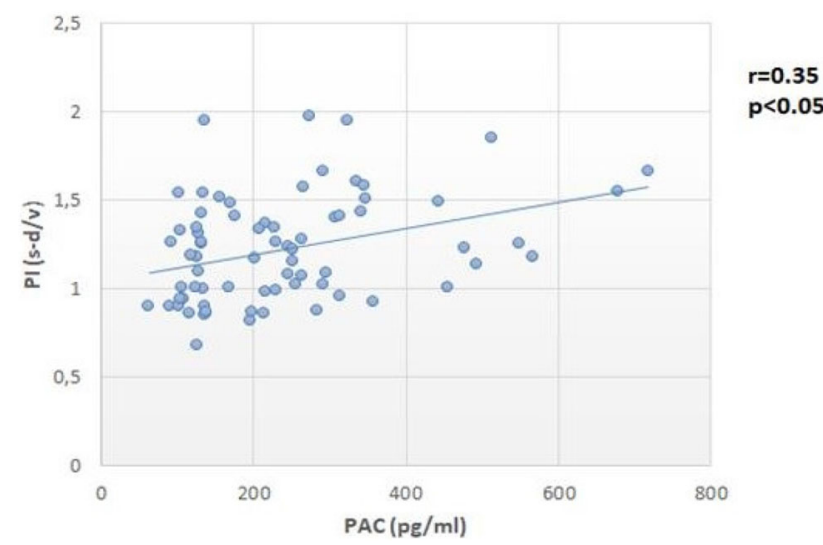

RELATIONSHIP OF ARTERIAL STIFFNESS PARAMETERS IN PATIENTS WITH ESSENTIAL ARTERIAL HYPERTENSION AND PRIMARY HYPERALDOSTERONISM

\author{
L. Petramala ${ }^{1}$, M. Mezzadri ${ }^{1}$, A. Concistrè ${ }^{1}$, \\ M. Soldini ${ }^{1}$, G. Iannucci ${ }^{2}$, C. Letizia ${ }^{1}$
}

${ }^{1}$ Department of Translational and Precision Medicine, "Sapienza" University of Rome, Rome, Italy ${ }^{2}$ Department of Clinical, Internal Medicine, Anesthesiology and Cardiovascular Sciences, Sapienza University of Rome, Rome, Italy

INTRODUCTION: Aldosterone is known to play important role in developing cardiovascular, metabolic, renal damage in hypertensive patients.

AIM: to evaluate arterial subclinical damage markers [24hours urinary albumin excretion (UAE) and arterial stiffness parameters by Pulse Wave Velocity (PWV)] in patients with Essential Hypertension (EH) and Primary Aldosteronism (PA), without overt organ damage.
METHODS: We consecutively enrolled 82 hypertensive subjects [37 males (mean age $48.9 \pm 13.3$ years) and 45 women (mean age $48.5 \pm 14.8$ years)], distinguished in two groups: $60 \mathrm{EH}$ patients and 22 PA patients [5 aldosterone-secreting adrenal adenoma (APA), 17 idiopathic aldosteronism (IHA)]; as control group we enrolled 37 normotensive subjects (NS).

RESULTS: PA group showed higher UAE excretion $(65.7 \pm 11.0 \mathrm{mg} / 24 \mathrm{~h})$ than $\mathrm{EH}$ and NS $(21.5 \pm 7.0 \mathrm{mg} / 24 \mathrm{~h}$, $9.2 \pm 15.0 \mathrm{mg} / 24 \mathrm{~h}$, respectively; $\mathrm{p}<0.02)$; in particular, APA patients showed higher UAE excretion than IHA $(111.8 \pm 56.0 \mathrm{mg} / 24 \mathrm{~h}$ vs $65.7 \pm 11.0 \mathrm{mg} / 24 \mathrm{~h} ; \mathrm{p}<0.001)$. In overall hypertensive population, UAE was positively correlated to PAC $(\mathrm{r}=0.25 ; \mathrm{p}<0.02)$ and AUR $(\mathrm{r}=0.3$; $\mathrm{p}<0.001)$. APA group showed significant increased values of Arterial Stiffness Index $(11.7 \pm 4.8 \mathrm{~m} / \mathrm{s}$; $\mathrm{p}<0.02)$ compared to IHA $(8.8 \pm 2.3 \mathrm{~m} / \mathrm{s}), \mathrm{EH}(8.3 \pm 3 \mathrm{~m} / \mathrm{s}) \mathrm{NS}$ $(7.2 \pm 1.7 \mathrm{~m} / \mathrm{s})$. Furthermore, APA patients showed significant reduced levels both of Subendocardial Variability Ratio (SEVR) $(104.8 \pm 25.7 \%)$ and Travel Time of reflected waves $(83.8 \pm 28.3 \mathrm{~ms})$, compared to $\mathrm{EH}$ (respectively $120.5 \pm 22.4 \%, 112.9 \pm 38.1 \mathrm{~ms} ; \mathrm{p}<0.05$ ) and NS (respectively $119.8 \pm 12 \%, 131.2 \pm 32 \mathrm{~ms} ; \mathrm{p}<0.05)$. Using multiple linear regression model, in overall hypertensive patients UAE value has been shown to predict Augmentation Index $(\beta=0.025 ; \mathrm{p}<0.01)$, SEVR $(\beta=-0.067 ; \mathrm{p}<0.03)$ and Arterial Stiffness $(\beta=0.021 ; \mathrm{p}<0.001)$; predictors of Travel Time of reflected waves were UAE $(\beta=-0.178$; $\mathrm{p}<0.01)$, triglycerides $(\beta=-0.139 ; \mathrm{p}<0.05), \operatorname{SBP}(\beta=-$ $1.001 ; \mathrm{p}<0.01)$, DBP $(\beta=-1.567 ; \mathrm{p}<0.01)$, age $(\beta=-$ $1.157 ; \mathrm{p}<0.01)$ and waist circumference $(B=-0.940$; $\mathrm{p}<0.03$ ) (Figure 3).

CONCLUSIONS: PA patients showed higher cardiovascular subclinical damage, evaluated by PWV, respect to $\mathrm{EH}$; in overall hypertensive population, UAE excretion had significant correlation with aldosterone behaviors, resulting the best marker of subclinical vascular remodeling.

Figure $3 . \quad r=0.51$

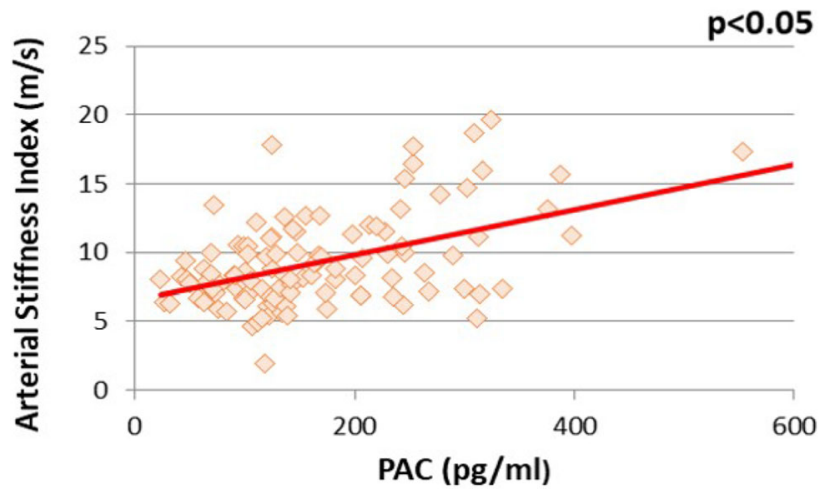




\section{DILATED CARDIOMYOPATHY, ELECTROCARDIOGRAPHIC CHANGES AND CORONARY-PULMONARY ARTERY FISTULA: AN UNUSUAL PRESENTATION OF PRIMARY ALDOSTERONISM IN THE COVID-19 ERA}

\author{
L. Marzano MD, MSc; A. Rebellato MD, PhD; \\ F. Ragazzo, MD; G. Scanelli, MD
}

Internal Medicine Unit, St. Bortolo General Hospital, U.L.S.S. 8 Berica-Veneto Region, 36100 Vicenza, Italy

INTRODUCTION: Primary aldosteronism is the most common cause of secondary hypertension. Elevated aldosterone levels have been associated with endothelial proliferation and pathological remodeling of the heart and arteries; however, coronary arterial abnormalities have never been reported in patients with primary aldosteronism. CASE REPORT: We report the case of a 53-year-old obese man (BMI $37 \mathrm{~kg} / \mathrm{m}^{2}$ ) with recent history of hypertension, fatigue and subjective dyspnoea who was admitted to our hospital after performing an echocardiography that showed hypertensive and ischemic heart disease in dilated phase. His laboratory results showed hypokalemia and an elevated aldosterone/renin ratio. After case confirmation, the abdominal CT scan showed a slight thickening of the left adrenal gland and a subcentimeter adenoma in the contralateral gland. Interestingly, that after oral potassium correction, the electrocardiogram revealed a symmetrical T-wave inversion and subsequently a coronary angiography detected a coronary-pulmonary fistula in the absence of significant stenosis. In the Italy's coronavirus pandemic full spread, we preferred to postpone the diagnosis of the lateralization of aldosterone hypersecretion by adrenal venous sampling and we started a mineralocorticoid receptor antagonist with a clinical follow-up of cardiovascular symptoms for the optimal treatment of coronary artery fistula. After about two months of follow-up, the patient remains asymptomatic with good blood pressure control and normal potassium levels.

CONCLUSIONS: Our case highlights a possible association between hypertensive patients with coronary artery fistulas and primary aldosteronism. We recommend clinicians and cardiologists to maintain a high level of suspicion of primary aldosteronism in these patients.

\section{PREVALENCE OF ENDOCRINE CAUSES OF HYPERTENSION: A RETROSPECTIVE STUDY FOCUSED ON SUBCLINICAL HYPERCORTISOLISM}

Aresta Carmen $^{1}$, Favero Vittoria ${ }^{1,2}$, Ripepi Federica ${ }^{2}$, Giovanelli Luca $^{1,2}$, Bilo Grzegorz Marek ${ }^{3,4}$, Pengo Martino $^{3}$, Parati Gianfranco ${ }^{3,4}$, Persani Luca ${ }^{1,2}$, Chiodini Iacopo ${ }^{1,2}$

${ }^{1}$ Department of Endocrinology and Metabolism, Istituto Auxologico Italiano IRCCS, Milan, Italy; ${ }^{2}$ Department of Clinical Sciences \& Community Health, University of Milan, Milan, Italy; ${ }^{3}$ Department of Cardiovascular, Neural and Metabolic Sciences, Istituto Auxologico Italiano IRCCS, Milan, Italy; ${ }^{4}$ Department of Medicine and Surgery, University of Milano-Bicocca, Milan, Italy

INTRODUCTION: Endocrine causes are involved in arterial hypertension (AH) pathogenesis in 5-10\% of cases. AIM: The aims of the present study are to evaluate in a hypertensive population at higher risk of secondary causes: (i) prevalence of endocrine hypertension (EH) and subclinical hypercortisolism (SH) (ii) clinical predictors of $\mathrm{EH}$ (iii) IS-correlated clinical parameters

METHODS: 47 patients (age 25-84 years) were selected on the basis of inclusion criteria (grade 3/resistant $\mathrm{AH}$; sudden new onset or unexpected worsening $\mathrm{AH}$; onset of $\mathrm{AH}<30$ years without risk factors; organ damage disproportionate to the grade/length of $\mathrm{AH}$ ) and exclusion criteria (specific signs/symptoms of endocrinopathy). In all patients we assessed blood pressure levels (systolic blood pressure SBP, diastolic blood pressure DBP, mean arterial pressure MAP, ambulatory blood pressure monitoring ABPM), cortisol after 1 mg-overnight-dexamethasone, ACTH, 24-hour urinary free cortisol, 24-hour urinary fractionated metanephrines, aldosterone, renin, IGF1, TSH, calcium, albumin, PTH.

RESULTS: EH was diagnosed in $51 \%$ of patients (primary aldosteronism 19\%, SH 17\%, pheochromocytoma 6\%, acromegaly $2 \%$, hyperthyroidism $2 \%$, hyperparathyroidism $2 \%)$. SBP, DBP, MAP levels and prevalence of non-dippers were higher in $\mathrm{EH}$ patients $(146 \pm 20 \mathrm{mmHg}$, $93 \pm 14 \mathrm{mmHg}, 111 \pm 15 \mathrm{mmHg}, 60.0 \%)$ than in the remaining patients $(135 \pm 12 \mathrm{mmHg}, 84 \pm 7 \mathrm{mmHg}$, $101 \pm 7 \mathrm{mmHg}, 9.1 \%, \mathrm{p}<0.05)$. EH diagnosis was independently associated with older age and non-dipping pattern (OR IC 95\%: 1.15, 1.013-1.302, $\mathrm{p}=0.031 ; 28.98$, 1.259-667.119, $\mathrm{p}=0.035$ ). SH patients showed higher levels of DBP and MAP $(89 \pm 5 \mathrm{mmHg}, 108 \pm 6 \mathrm{mmHg})$ compared with the essential AH patients $(84 \pm 7 \mathrm{mmHg}$, $101 \pm 7 \mathrm{mmHg}, \mathrm{p}<0.05)$. 
CONCLUSIONS: In a hypertensive population at higher risk of secondary causes, prevalence of EH was 51\%; nondipping pattern and older age were clinical predictors of $\mathrm{EH}$; prevalence of SH was 17\%; blood pressure levels were higher in SH patients compared with essential AH patients.

\section{ADRENAL VENOUS SAMPLING IN PRIMARY ALDOSTERONISM: THE ROLE OF PRE-TEST COMPUTED TOMOGRAPHY ANGIOGRAPHY}

\author{
Alessandra Violet Bacca, Giulia Aloisi, Alessio \\ Balletti, Nicola Riccardo Pugliese, Stefano Taddei
}

\section{Hypertension Unit, Department of Clinical and Experimental Medicine University of Pisa, Pisa, Italy}

INTRODUCTION: Primary aldosteronism (PA) is the most common cause of endocrine hypertension. It is pivotal to distinguish bilateral idiopathic hyperaldosteronism (IHA) from aldosterone-producing adenoma (APA) because surgical removal of the latter can effectively treat PA. Current international guidelines consider adrenal venous sampling (AVS) as the gold standard for diagnosing APA. Pre-test adequate imaging reconstruction of adrenal vein drainage by computed tomography $(\mathrm{CT})$ angiography can improve AVS success.

AIM: to evaluate the AVS performance comparing the selectivity Index of patients who underwent pre-test AngioCT versus a traditional AVS approach with basal CT.

METHODS: This was a retrospective observational study. We included all patients who underwent AVS in the Department of Internal Medicine at Pisa University Hospital between January 2010 and February 2020. We reviewed the medical records to obtain information regarding clinical characteristics, laboratory findings for the case detection and diagnosis of PA, computed imaging scans, AVS results and surgical data. Successful AVS was defined as an adrenal vein to inferior vena cava cortisol ratio $>2$ (selectivity index).

RESULTS: We collected data of 124 AVS and analyzed complete records of 97 patients (59 males and 38 females, median age 48.3 yrs): $37.1 \%(36 / 97)$ executed pre-test Angio CT scan and 62.8\% (61/97) only basal CT. Right AVS failed in $53.6 \%$ (52/97) of cases with pre-test angioCT in $32 \%(17 / 52, \mathrm{P}=0.3 \mathrm{NS})$. Likewise, left AVS failed in $10.3 \%$ (11/97) of cases with pre-test angioTC in $54 \%(6 / 11, \mathrm{P}=0.2 \mathrm{NS})$;

CONCLUSIONS: The difficulty of recognizing or cannulating the right adrenal vein is the most frequent cause of AVS failure, due to small length and/or anatomic variations. The pre-test Angio-CT allows an accurate reconstruction of the right adrenal vein before AVS and may help to improve the success rate of the invasive procedure.

\section{IN VIVO URINARY EXOSOMAL 11B-HSD2 EXPRESSION IN A FAMILY WITH APPARENT MINERALOCORTICOID EXCESS}

Domenica De Sanctis, Giulia Sartori, Simonetta Friso, Mariangela Veneri, Francesca Morandini, Silvia Udali, Annalisa Castagna, Patrizia Pattini, Chiara Zaltron, Elisa Danese, Olivieri Oliviero, Francesca Pizzolo

1. Department of Medicine, Internal Medicine Section, University of Verona; 2. Department of Neurosciences, Biomedicine and Movement Sciences, Clinical Biochemistry Section, University of Verona

INTRODUCTION: Apparent mineralocorticoid excess (AME) is an autosomal recessive disorder caused by the $11 \beta$-hydroxysteroid dehydrogenase type 2 (11 $\beta$-HSD2) enzyme deficiency. Traditionally, $11 \beta-\mathrm{HSD} 2$ activity is assessed by measuring the cortisol metabolite ratio (tetrahydrocortisol/tetrahydrocortisone, THF $+5 \alpha \mathrm{THF} / \mathrm{THE}$ ) in $24 \mathrm{~h}$ urine collection. Urinary exosomes, vesicles derived from the renal tubule, are a useful tool to investigate rare proteins and mRNA in diseases. It is not known whether urinary exosomal expression HSD11B2 is related to $\mathrm{THF}+5 \alpha \mathrm{THF} / \mathrm{THE}$, and to mutation status in patients with AME.

AIM: to isolate 11 $\beta$-HSD2 mRNA from urinary exosomes in a family with AME, and to investigate the relationship with either THF $+5 \alpha \mathrm{THF} / \mathrm{THE}$ ratio and the A221G $(662 \mathrm{C}>\mathrm{G})$ variant.

METHODS: among the A221G carriers (two homozygous probands, two heterozygous parents, four heterozygous relatives) and four wild-type family members, THF +5 $\alpha \mathrm{THF} / \mathrm{THE}$ ratio, hormonal and biochemical parameters were measured. Urinary exosomes were extracted from morning urine by precipitant reagent and centrifugation. Exosomal RNAs were isolated and pre-amplified with specific kit and primers. Absolute quantification of HSD11B2 mRNA (copies/ $\mu$ l) was assessed by Droplet Digital PCR (ddPCR) using B2M (microglobulin2-beta) gene as the housekeeping.

RESULTS: $11 \beta$-HSD2 mRNA was detectable in 9 out of 12 samples. Expression of HSD11B2 mRNA varied according to the $662 \mathrm{C}>\mathrm{G}$ genotype so that the two probands showed the highest levels $(169 \pm 72)$, the four heterozygous intermediate levels $(52.54 \pm 53.85)$, and the three wild-type subjects had the lowest levels $(8 \pm 5.22)$. The $11 \beta$-HSD2 expression related to the hypertensive 
status among heterozygous subjects. Among the 9 samples where it was detectable, the HSD11B2mRNA correlated inversely with their renin and aldosterone serum concentrations, and slightly positively with $\mathrm{THF}+5 \alpha \mathrm{THF} / \mathrm{THE}$ ratio.

CONCLUSIONS: HSD11B2 mRNA is detectable in urinary exosomes and its abundance relates to $11 \beta-\mathrm{HSD} 2$ enzyme activity as estimated by the THF $+5 \alpha \mathrm{THF} / \mathrm{THE}$ ratio. The HSD11B2 exosomal expression is coherent with the different $662 \mathrm{C}>\mathrm{G}$ genotypes within AME family members, and clearly paralleled with the hypertensive status among heterozygous subjects. Exosomal HSD11B2 mRNA is a useful tool to investigate $11 \beta$-HSD2 deregulation in relation to hypertension.

\section{URINE METABOLITES OF CORTISOL CORRELATES WITH AMBULATORY BLOOD PRESSURE VALUES IN OBESE CHILDREN}

\author{
A. Tagetti ${ }^{1}$, E. Danese ${ }^{2}$, M. Pucci ${ }^{2}$, P. Minuz ${ }^{1}$, \\ F. Antoniazzi ${ }^{3}$, C. Maffeis ${ }^{3}$, C. Fava ${ }^{1}$
}

1. Department of Medicine, University of Verona, Italy; 2. Department. of Neuroscience, Biomedicine and Movement Sciences, University of Verona, Italy; 3. Department of Surgery, Dentistry, Paediatrics and Gynecology, University of Verona, Italy

INTRODUCTION: Obesity is considered a pseudoCushing state and often elevated levels of cortisol and its metabolites can be found; however, the relationship between cortisol metabolites and blood pressure (BP) in obese children is understudied. 11- $\beta$-hydroxysteroid dehydrogenase type 2 (11 $\beta$-HSD-2) catalyses the reversible conversion of physiological glucocorticoids (tetrahydrocortisol, THF and allo-tetrahydrocortisol, allo-THF) to inactive products (tetrahydrocortisone, THE). An increase in the THFs/THE ratio reflects 11 $\beta$-HSD-2 impairment and has been associated with BP elevation. Ambulatory blood pressure monitoring (ABPM) provides an accurate measurement of BP during $24 \mathrm{~h}$.

AIM: We aimed to investigate the relationship between BP measured by ABPM, urinary cortisol and its metabolites, in a sample of obese children.

METHODS: 50 obese children aged between 8 and 17 years (mean age $11.5 \pm 3.3$ years, 66\% males), were included in the present study. Children underwent ABPM. Cortisol, sodium, THF, allo-THF and THE were measured on urinary 24-hour collection. Cortisol urinary metabolites were quantified by liquid chromatography tandem mass spectrometry (Nexera Shimadzu, 4500 MD Sciex).
RESULTS: TheTHF + alloTHF/THE ratio negatively correlates with 24-hours urinary sodium excretion $(p=-$ $0.346 ; r=0.015$ ) and to SBP and DBP load (percentage of measure of PAS $>130 \mathrm{mmHg}$ or percentage of PAD $>80$ $\mathrm{mmHg}$ ) (respectively $\mathrm{r}=0.283 \mathrm{p}=0.047$ and $\mathrm{r}=0,284$; $p=0.046$ ). No correlation between THFs/THE ratio and daytime or night-time SPB or DBP were found. The sum of urinary cortisol and its metabolites, a gross index of relative excess of cortisol, is directly correlated with SBP load $(\mathrm{r}=0.355 ; \mathrm{p}=0.029)$.

CONCLUSIONS: In our sample of obese children, both the impaired activity of the 11-beta-HSD-2 and the amount of cortisol and its metabolites excreted by urine are associated with an excessive 24-h BP load. Reduced urinary sodium excretion mediated by increasing of THF/THE ratio could be involved.

\section{PREGNANCY OUTCOME WITH HELLP SYNDROME}

Piera Altieri ${ }^{1}$, Claudio Borghi ${ }^{1}$, Nicola Rizzo ${ }^{1}$, Eugenio Roberto Cosentino ${ }^{1}$, Crescenzio Bentivenga ${ }^{1}$

\section{${ }^{1}$ Azienda Ospedaliero Universitaria Sant'Orsola Malpighi, Bologna, ITALY}

INTRODUCTION: The HELLP Syndrome (HS) is a serious complication in pregnancy characterized by Hemolysis, Elevated, Liver enzymes, Low Platelets and also by cephalea, visual disturbances, epigastralgia and, in many cases, a risk for impaired renal function. PreEclampsia (PE) is a condition implying hypertension, proteinuria and/or associated alterations. Generally, it occurs after the 20th week of pregnancy in previously normotensive, non proteinuric women and it is reversible in postpartum within 6-12 weeks.

AIM: The purposes of this study are: (1) to verify the impact of HS in women affected by the aforementioned syndromes in the areas of Bologna and San Lazzaro di S., and to report a longitudinal survey about the main outcomes of the children born to affected patients in the period between 2004 and 2018; (2) to look for a correlation between mothers afflicted by those syndromes and the development of Metabolic Syndrome (MS), hyperuricemia, or cardiovascular disease (CD) in their children and to ascertain whether the paternal age or potential comorbidities can lead to these pathologies in the partner and to any predispositions and pathologies in the child.

METHODS: we have considered all the useful parameters-as well as the bio humoral ones-which can determine the diagnosis of HELLP or PE syndrome, and we have created an appropriate database built on medical 
records. Furthermore, retrospective research on primary care pediatrician and on all completed pregnancies was carried out using a checklist containing measures of outcomes and of the main cardiovascular risk indicators. During the period considered by this study, the results revealed an distinct increase in the number of women affected by these syndromes. This could be a consequence of a greater accuracy in diagnosis, or because the average age of pregnant women has risen as compared to the past, or because of the increased use of assisted reproductive techniques.

RESULTS: According to the available data, it seems that there is no correlation between the mother's pathology and the development of MS in children nor a predisposition to cardiovascular diseases, at least in the period we are considering, which is normally characterized by a low incidence of cardiovascular problems. Lastly, it would seem very suggestive that the fathers' reproductive age can reverberate negatively on the partners who are affected in a tendentially higher degree. Similarly to what emerged from the data of children born to affected mothers, no predisposition to these syndromes appears in children during their infant-youth age. In light of these findings, it is expected that these patients shall have a more extensive and rigorous screening.

\section{PRIMARY ALDOSTERONISM AND OBSTRUCTIVE SLEEP APNEA: A MULTICENTER MULTI-ETHNIC CROSS-SECTIONAL STUDY}

Fabrizio Buffolo ${ }^{1}$, Qifu Li ${ }^{2}$, Silvia Monticone ${ }^{1}$, Daniel A. Heinrich ${ }^{3}$, Alessio Mattei ${ }^{4}$, Jacopo Pieroni ${ }^{1}$, Mei $\mathrm{Mei}^{2}$, Shumin Yang ${ }^{2}$, Ya-Hui Hu${ }^{5}$, Mei-Chen Yang ${ }^{6}$, Chiara Sabbadin ${ }^{8}$, Francesca Pizzolo ${ }^{9}$, Gilberta Giacchetti $^{7}$, Francesco Fallo ${ }^{8}$, Franco Veglio ${ }^{1}$, Martin Reincke $^{3}$, Vin-Cent $\mathrm{Wu}^{10}$, Paolo Mulatero ${ }^{1}$

${ }^{1}$ Division of Internal Medicine and Hypertension Unit, Department of Medical Sciences, University of Torino, Torino, Italy; ${ }^{2}$ Department of Endocrinology, the First Affiliated Hospital of Chongqing Medical University, Chongqing, China; ${ }^{3}$ Medizinische Klinik und Poliklinik IV, Klinikum der Universität, Ludwig-Maximilians-Universität München, Munich, Germany; ${ }^{4}$ Cardiovascular and Thoracic Department, Città della Salute e della Scienza, Turin, Italy; ${ }^{5}$ Division of Endocrine and Metabolism, Department of Internal Medicine, Taipei Tzu Chi Hospital, The Buddhist Medical Foundation, Taipei, Taiwan; ${ }^{6}$ Division of Pulmonary Medicine, Department of Internal Medicine, Taipei Tzu Chi Hospital, The Buddhist Medical Foundation, Taipei, Taiwan;

${ }^{7}$ Division of Endocrinology, Polytechnic University of Marche, Ancona, Italy; ${ }^{8}$ Department of Medicine, DIMED, Internal Medicine 3, University of Padua, Padova, Italy; ${ }^{9}$ Department of Medicine, Unit of Internal Medicine, University of Verona, Verona, Italy; ${ }^{10}$ Division of Nephrology, Department of Internal Medicine, National Taiwan University Hospital, Taipei, Taiwan

INTRODUCTION: despite current evidences are limited, 2016 Endocrine Society guideline recommends screening for primary aldosteronism (PA) in all individuals with arterial hypertension and obstructive sleep apnoea (OSA). AIM: We performed a multicentre, multi-ethnic, crosssectional study, designed to investigate the prevalence of PA in patients with OSA and the prevalence of OSA in patients with PA.

METHODS: we screened 203 patients with OSA (102 of Caucasian and 101 of Chinese ethnicity) for PA. In case of positive screening, patients underwent confirmatory test for PA and subtyping diagnosis. 207 patients with PA (104 Caucasians, 100 Chinese and 3 of African descent) were screened for OSA by cardio-respiratory polygraphy.

RESULTS: $8.9 \%$ of patients with OSA had confirmed PA diagnosis $(11.8 \%$ in Caucasian and $5.9 \%$ in Chinese patients). The prevalence of PA in patients without other indications for PA screening, other than OSA diagnosis, was very low $(1.5 \%)$. The prevalence of OSA was $67.6 \%$ (64.4\% in Caucasian and $70.0 \%$ in Chinese patients). OSA was more prevalent in PA patients with idiopathic hyperaldosteronism than aldosterone-producing adenoma (75.8\% vs $59.2 \%, \mathrm{p}=0.031)$. We observed a significant correlation between aldosterone levels and apnea/hypopnea index in Caucasian group with PA $\left(\mathrm{R}^{2}=0.360, \mathrm{p}=0.013\right)$, but not in the Chinese group with PA. Similarly, multinomial logistic regression confirmed a significant association between plasma aldosterone and moderate to severe OSA in Caucasian patients (OR 1.002, $\mathrm{p}=0.002)$, independently of the main confounding factors. No association was present in Chinese patients with PA.

CONCLUSIONS: patients with OSA do not have an increased risk of PA, challenging the current recommendation of the Endocrine Society guideline for screening all patients with OSA for PA, independently of hypertension severity. However, OSA is frequent in patients with PA and aldosterone level can worsen OSA severity in Caucasian patients. 


\section{SCREENING OF PRIMARY ALDOSTERONISM WITH A NOVEL RENIN-ANGIOTENSIN- ALDOSTERONE SYSTEM TRIPLE-A ANALYSIS}

Jacopo Burrello ${ }^{1}$, Fabrizio Buffolo ${ }^{1}$, Oliver Domenig ${ }^{2}$, Martina Tetti ${ }^{1}$, Alessio Pecori ${ }^{1}$, Silvia Monticone ${ }^{1}$, Marko Poglitsch $^{2}$, Paolo Mulatero ${ }^{1}$

(1) Division of Internal Medicine and Hypertension, Department of Medical Sciences, University of Turin, Turin, Italy. (2) Attoquant Diagnostics, Vienna, Austria

INTRODUCTION: Primary aldosteronism (PA) is a frequent cause of secondary hypertension and its screening is expected to become a routine evaluation in patients with hypertension. The interference of antihypertensive medications with the Aldosterone-to-Renin-Ratio (ARR) is a major confounder during screening testing. Renin-angiotensin-aldosterone-system (RAAS) triple-A analysis is a novel liquid chromatography/tandem mass spectrometry diagnostic assay that allows simultaneous quantification of aldosterone, equilibrium angiotensin I (eqAngI) and angiotensin II (eqAngII).

AIM: to assess the reliability of RAAS triple-A analysis for PA screening.

METHODS: We evaluated the diagnostic performance of the Aldosterone-to-AngII-Ratio (AA2R) and five renin based diagnostic ratios, differing in methods to determine aldosterone levels and renin activity, either based on chemiluminescence or radioimmunoassay.

RESULTS: We enrolled a cohort of 110 patients with hypertension and suspected PA referred to a single hypertension unit (33 patients with confirmed PA and 77 with essential hypertension). All ratios showed comparable areas under the curves ranging between 0.924 and 0.970 without significant differences between each other. The evaluation of the AngII-to-AngI ratio revealed persistent ACE inhibitor intake in some patients as cause for suppressed renin-based diagnostic ratios, while AA2R remained unaffected, allowing a AA2-R-based PA screening in presence of ACE inhibitor. The optimal cutoff value for the AA2R was $6.6[(\mathrm{pmol} / \mathrm{L}) /(\mathrm{pmol} / \mathrm{L})]$ with a sensitivity and specificity of $90 \%$ and $93 \%$, respectively, non-inferior to the ARR while pointing to the potential for an interference free application in patients under ACE-inhibitor therapy.

CONCLUSIONS: This study shows for the first time the accuracy and reliability of RAAS triple-A analysis for the screening of PA, even in the presence of therapy with ACE inhibitors. Combining information on drug efficacy and compliance monitoring with PA screening, this method might have a significant impact on the overall performance of PA screening.
Blood Pressure Measurement

\section{WHICH IS THE OPTIMAL CUFF FOR AN ACCURATE BLOOD PRESSURE MEASUREMENT IN THE VERY OBESE SUBJECTS?}

Paolo Palatini, Elisabetta Benetti, Claudio Fania, Andrea Ermolao, Paolo Spinella, Francesca Battista, Andrea Gasperetti, Francesca Saladini

Department of Medicine, University of Padova, Padua, Italy

INTRODUCTION: Rectangular (cylindrical) cuffs and bladders are currently used for blood pressure (BP) measurement at the upper arm. However, large arms have a conical shape which make cylindrical cuffs potentially unsuitable.

AIM: to study the shape of the arm in people with severe obesity and to investigate the effect of the shape of the cuff on BP measurement in these subjects.

METHODS: We compared cylindrical and tronco-conical cuffs of appropriate size in 79 obese subjects with upper arm mid-circumference $>40 \mathrm{~cm}$ and 79 subjects of control (arm mid-circumference $<33 \mathrm{~cm}$ ). The upper arm was considered either as a single truncated cone (model 1) or as the sum of two tronco-conical shapes, with bases at the proximal and middle arm circumference, respectively (model 2). We measured the frustum slant angle of the proximal (upper $\alpha$ ) and distal (middle $\alpha$ ) truncated cones, if the difference between "upper $\alpha$ " and "middle $\alpha$ " was equal to zero, the upper arm can be assimilated to a single truncated cone (Figure 4a), if the difference was positive, it means that the distal half of the arm was more conical than the proximal half (Figure $4 b$ ).

RESULTS: In the Obese, upper $\alpha$ was greater than middle $\alpha$, whereas in the Nonobese the two angles were similar $(p<0.0001$ versus Obese for both $\alpha)$. In the Obese, the cylindrical cuff overestimated BP measured with the tronco-conical cuff by $4.3 \pm 5.4 / 3.1 \pm 4.7 \mathrm{mmHg}$, whereas in the Nonobese slight discrepancies were found between the two cuffs ( $\mathrm{p}<0.0001 /<0.0001$ versus Obese). In the whole study sample, the difference between the "upper $\alpha$ " and "middle $\alpha$ " was correlated with systolic BP $(\mathrm{p}=0.0008)$ and diastolic $(\mathrm{p}<0.0001)$ BP discrepancies. In a multivariable linear regression, the difference between the "upper $\alpha$ " and "middle $\alpha$ " was an independent predictor of the between-cuff BP differences in obese men ( $p=0.004 / 0.0007)$ but not in obese women.

CONCLUSIONS: In very obese people, the tronco-conical shape of the upper-arm is more pronounced on the 
distal than the proximal half, a feature that amplifies the BP measurement error when cylindrical cuffs are used.

Figure 4.

Figure a

Circonf $<33 \mathrm{~cm}$

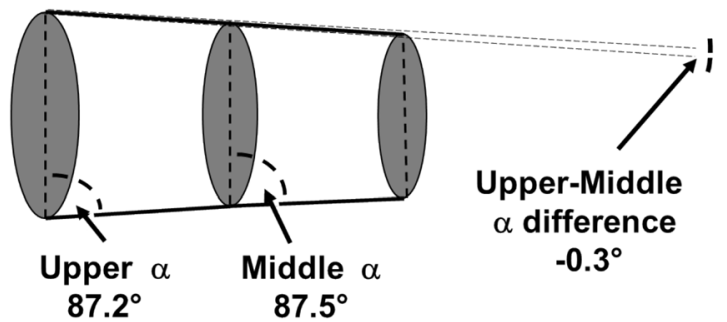

Figure $b$

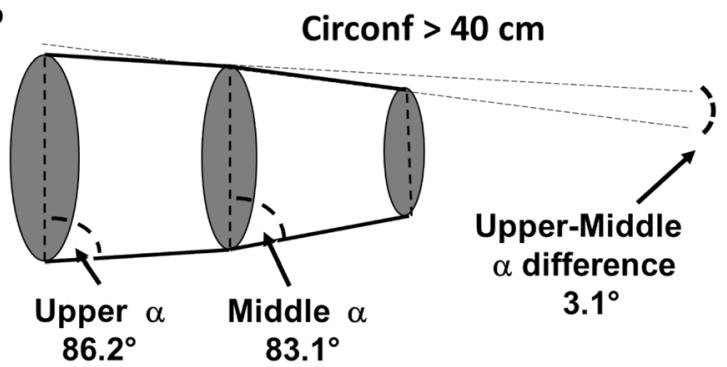

\section{LOW NIGHT-TIME BLOOD PRESSURE IS A KEY PROGNOSTIC FACTOR IN OLDER EXTREME DIPPERS FROM THE ABP-INTERNATIONAL STUDY}

P Palatini ${ }^{1}$, G Reboldi ${ }^{2}$, F Angeli ${ }^{2}$, L Beilin $^{3}$, $\mathrm{K}$ Eguchi $^{4}$, Y Imai ${ }^{5}, \mathrm{~K}$ Kario $^{4}$, T Ohkubo ${ }^{5}$,

S Pierdomenico $^{6}$, F Saladini ${ }^{1}$, JE Schwartz ${ }^{7}$, L Wing ${ }^{8}$, P Verdecchia ${ }^{9}$

${ }^{1}$ Dipartimento di Medicina, Università degli Studi di Padova, Padova, ITALY, ${ }^{2}$ Università di Perugia, Perugia, Perugia, ITALY, ${ }^{3}$ University of Western Australia, Perth, Perth, AUSTRALIA, ${ }^{4}$ Jichi University, Tochigi, Tochigi, JAPAN, ${ }^{5}$ Tohoku University, Sendai, Sendai, JAPAN, Università di Chieti, Chieti, ITALY, ${ }^{7}$ Columbia University, New York, New York, USA, ${ }^{8}$ Flinders University, Adelaide, Adelaide, AUSTRALIA, ${ }^{9}$ Hospital of Assisi, Assisi, ITALY

INTRODUCTION: In a previous analysis of the ABPInternational study we showed that extreme dipping in the elderly carries an increased risk of cardiovascular events (CVE) similar to that of reverse dipping. However, the reason for this association remained uncertain.

AIM: The present analysis was conducted to test the hypothesis that the main factor accounting for the risk associated with extreme dipping is a low night-time blood pressure (BP).

METHODS: We included 10,868 participants (53\% men) aged $53 \pm 15$ years (hypertensives, $81 \%$ ) enrolled in 8 prospective studies in Australia, Italy, Japan, and U.S.A. Using the systolic BP nocturnal decline (in percent) we identified 3 groups: dippers ( $>10-20 \%)$, non-dippers $(\leq$ $10 \%$ ), and extreme dippers ( $>20 \%)$. Extreme dippers were further splitted into two groups, one with low night-time systolic BP $(<120 \mathrm{mmHg}$, ED-low-NBP) and one with high night-time $\mathrm{BP}(\geq 120 \mathrm{mmHg}$, ED-high-NBP). The association between dipping category and CVE was estimated as a function of age ( $\leq 70$ or $>70$ years) using Cox models adjusted for average 24-hour systolic BP and traditional risk factors.

RESULTS: During $6.6 \pm 4.0$ years of follow-up there were a total of 829 CVE (168 fatal). Among the older participants, there was a significant increase in risk for both non-dippers and extreme dippers, compared to dippers. However, among the extreme dippers a significant increase in risk was found only in ED-low-NBP. The HRs $(95 \% \mathrm{CI})$ were $1.57(1.13-2.20)$ in the non-dippers $(p=0.008)$, 2.59(1.33-5.09) in the ED-low-NPB $(\mathrm{p}=0.005)$, and 1.15(0.49-2.68) in the ED-high-NBP $(\mathrm{p}=0.75)$. If the night-time systolic BP cut-off was set at $110 \mathrm{mmHg}$, the HR in the ED-low-NBP rose to 3.44 (1.20-9.87). No increase in risk of CVE was observed among the extreme dippers $\leq 70$ years for both night-time BP cut-offs irrespective of whether night-time BP was low or high ( $p>0.59$ for all groups).

CONCLUSIONS: These data confirm that the prognostic value of extreme dipping is heavily dependent on age. However, a key factor in predicting prognosis is the level of night-time BP. Prolonged exposure to low BP during the night may be harmful due to cerebral and cardiac hypoperfusion.

\section{SLEEP APNOEA AND ARTERIAL REDUCED COMPLIANCE INDUCE BOTH AN INCREASED BLOOD PRESSURE TOTAL REACTIVITY TO LABORATORY STRESS}

P. Nazzaro, G. Schirosi, A. Nardecchia, M. Contini, L. De Benedittis, G. Aceto, A.M. Papagni

Dept of Neurosciences, Unit of Hypertension "AM. Pirrelli", "Aldo Moro" University of Bari, Italy

INTRODUCTION: Several studies showed that sleep apnoea (SA) independently enhances the cardiovascular risk but very few investigated its impact on blood pressure 
(BP) reactivity to stressful task in grade 1 hypertensives with increased arterial stiffness.

METHODS: After medical visit and detection of risk factors for SA (Lausanne test: LSAs), 110 hypertensives $(132 \pm 5 / 82 \pm 6)$, with similar age, office SBP/DBP, BMI and metabolic state, underwent ABPM with actigraphy and skin oximetry to check sleeping time (SleepT), desaturation time (m'<90\%) and index (ODI).They underwent arterial tonometry (PWVcf) and laboratory stress session composed by Color Word Stroop test (5'), ability to car driving basic (5') and skilful (5') videogame, alternated with baseline-recovery phases (10'). SBP/DBP was taken every min. and total stress response was measured as "area-under-the-curve" = value $\mathrm{x}$ time (auc).

RESULTS: Patients, in monotherapy with ACEi/ARB, in order of SA and arterial stiffness, were divided in 34 controls (SA-PW-), 33 with SA only (SA + PW-) and 43 with both SA and arterial stiffness $(\mathrm{SA}+\mathrm{PW}+)$. They presented similar diurnal $(131 \pm 4 / 78 \pm 5$ vs $132 \pm 4$ / $79 \pm 6$ vs $132 \pm 3 / 79 \pm 5$ ) but different nocturnal SBP/ DBP $\quad\left(117 \pm 4 / 69 \pm 9 \quad\right.$ vs $\quad 122 \pm 2 / 74 \pm 10^{* * *} \quad$ vs $124 \pm 7 / 75 \pm 9 * * *)$ and nocturnal SBP fall $(11 \pm 2$ vs $7 \pm 3^{* * *}$ vs $\left.6 \pm 6^{* * *}\right)$.

\begin{tabular}{|l|l|l|l|l|l|l|}
\hline pts/var & SleepT & LSAs & $m^{\prime}<90 \%$ & ODI & PWVcf & SBPauc \\
\hline SA-PW- & $431 \pm 84$ & $3.5 \pm 1.5$ & $27 \pm 18$ & $6.5 \pm 5.3$ & $8.8 \pm 0.8$ & $6613 \pm 177$ \\
\hline SA+PW- & $368 \pm 52^{* * *}$ & $11.1 \pm 2.1^{* * *}$ & $120 \pm 52^{* * *}$ & $20.2 \pm 5.1^{* * *}$ & $9.1 \pm 0.7$ & $6734 \pm 245^{*}$ \\
\hline SA+PW+ & $348 \pm 76^{* * *}$ & $11.9 \pm 1.9^{* * *}$ & $138 \pm 38^{* * *}$ & $21.1 \pm 3.9^{* * *}$ & $12.1 \pm 2.5^{* * * \wedge \wedge \wedge}$ & $6938 \pm 195^{* * * \wedge \wedge \wedge}$ \\
\hline
\end{tabular}

CONCLUSIONS: The findings show that SA is associated to increased functional BP laboratory emotional stress response and that this is magnified in patients with arterial stiffness. Quality of sleep, and its related disturbances, should be a critical part of the exam in hypertensives and amelioration of technology of the ambulatory blood pressure monitoring is expected.

\section{THE RELATIONSHIP BETWEEN THE BLOOD PRESSURE PROFILE AND THE MORNING SURGE IN CAUCASIAN ELDERLY HYPERTENSIVE OUTPATIENTS}

\author{
Reffo $\mathrm{A}^{1}$, Patetta $\mathrm{L}^{1}$, Giantin $\mathrm{V}^{1}$, Trevisan $\mathrm{C}^{1}$, Curreri \\ $C^{1}$, Costacurta $A^{1}$, Benetazzo $C^{1}$, Sergi $G^{1}$ \\ ${ }^{1}$ Clinica Geriatrica, Department of Medicine, Azienda \\ Ospedale-Università di Padova
}

INTRODUCTION: the ambulatory blood pressure monitoring provides an evaluation of circadian variability of blood pressure. Blood pressure profile (BPP) is considered a reliable prognostic indicator for cardiovascular outcomes, instead the prognostic significance of morning surge (MS) is more controversial. Some recent studies have shown that a blunted-MS (considered as the lowest decile of MS) was associated with a worse cardiovascular risk in hypertensive patients. Fujiwara et al. tried to explain such a result with a connection between a blunted-MS and a reverse dipper pattern. At the best of our knowledge it has not yet been investigated this association in Caucasian elderly hypertensive subjects.

METHODS: we considered hypertensive patients referring to the hypertension center of the Clinica Geriatrica, Azienda Ospedale-Università di Padova. We took blood pressure measurements with a gallium sphygmomanometer in the supine position and three times, at least, in standing position. Then we performed a 24-hour ABPM with a validated device. We divided the study patients according to BPP and MS (considered in deciles). We used the multivariable logistic regression to establish the effect of other factors on the relationship of interest.

RESULTS: the study included 425 patients (274 females, $64.5 \%$ ), with a mean age of $75.8 \pm 7.1$ years. Reverse and non-dipper patients were older than subjects with other BPPs $(\mathrm{p}=0.001)$. The majority of reverse dippers $(88 \%)$ had a circadian profile of 24-hour hypertension versus $54.3 \%$ of extreme dippers $(\mathrm{p}<0.001)$. We noticed significant differences in MS among BPP groups: a bluntedMS represented about a quarter of reverse dipper subjects $(25.3 \%)$ versus $5.7 \%$ of extreme dipper subjects $(\mathrm{p}<0.001)$. We found a significant correlation between the change in systolic blood pressure (SBP) during night time and morning surge $(\beta=0.40, p<0.001$; Figure 5$)$. Then we estimated a significantly augmented probability to show a blunted-MS in reverse dippers than in dippers $(\mathrm{OR}=9.68$, IC 95\% = 3.52-26.60; $\mathrm{p}<0.001$; Table 1$)$.

CONCLUSIONS: the present study shows that a bluntedMS is more prevalent in reverse dippers. Previous works described a link between blunted-MS and a worse cardiovascular prognosis. Kario K. hypothesized that this evidence could be explained with a pathological alteration of the autonomic nervous system, both in a hyper- or in a hypo-reactivity way, affecting the control of blood pressure. Further study is needed to clarify the pathophysiological mechanisms of this relationship and its prognostic significance. 
Figure 5.

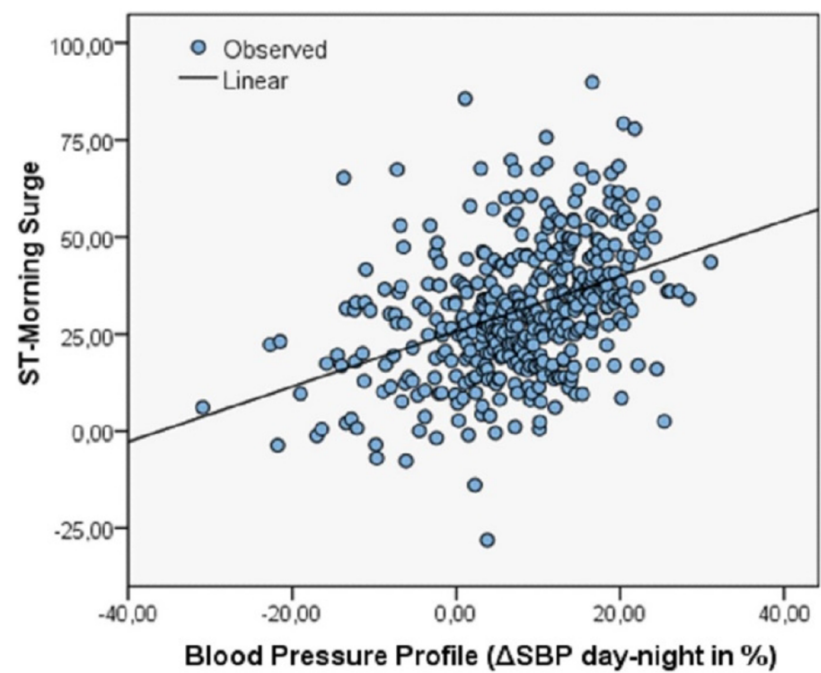

Table 1. Multiple regression analysis between "Blood Pressure Profile" (dipper: reference) e ST-Morning Surge.

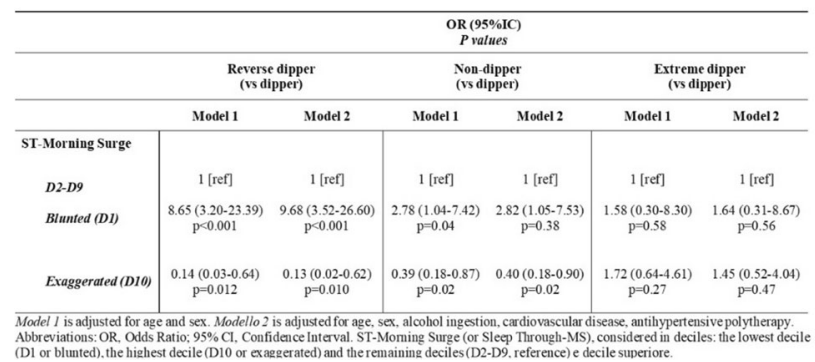

\section{PREVALENCE AND CORRELATES OF ISOLATED NOCTURNAL HYPERTENSION}

\author{
G. Mule' (1), L. Guarino (1), V. Cacciatore (1), \\ E. Mancia(1), A. Ferotti, N. Sinatra (1), F. Vaccaro (1), \\ M. Guarneri (1), A. Sorce (1), S. Cottone (1)
}

(1) ESH Hypertension Excellence Centre and Nephrology Unit - Department of Health Promotion, Mother and Child Care, Internal Medicine and Medical Specialties University of Palermo

INTRODUCTION: Isolated nocturnal hypertension (INH) represents a peculiar phenotype that can only be identified by 24-hour blood pressure monitoring. It is characterized by elevated night-time $\mathrm{BP}(>120$ and/or $70 \mathrm{mmHg})$ in the presence of normal daytime BP. The nocturnal BP values represent a less variable measure compared to the diurnal values and their eventual prognostic value remains unchanged also in the subjects pharmacologically treated, thus being able to become a marker of cardiovascular risk. METHODS: 1340 patients consecutively belonging to our Regional Referral Centre for Arterial Hypertension were enrolled and subjected to anamnesis and objective examination, routine laboratory investigations and 4-hour BP monitoring.

RESULTS: The clinical diastolic BP and the average systolic and diastolic values obtained by ABPM of $24 \mathrm{~h}$, of the day-time and night-time were greater in the group with INH compared to those of normotensive patients. At the univariate logistic regression analysis, the variables significantly associated with the presence of INH were male, metabolic syndrome, albuminuria and eGFR, considered both as continuous variables, as dichotomous variables.

CONCLUSIONS: The results of our study confirm that isolated nocturnal hypertension is a pressure phenotype that is far from rare, also and above all in subjects with clinical BP values within the normal range. Having identified some indices of early renal impairment, such as a slight increase in albuminuria and a moderate decrease of glomerular filtration, and the presence of metabolic syndrome, as characteristics associated with INH, can be useful for selecting, especially among patients clinically normotensive, those who must preferentially undergo 24-hour blood pressure monitoring in order to early recognize the cardiorenal risk associated with it.

\section{SCREENING FOR ATRIAL FIBRILLATION DURING AUTOMATED BLOOD PRESSURE MEASUREMENT AMONG PEOPLE ADMITTED TO AN INTERNAL MEDICINE WARD}

\author{
Santoni E, Calandri C, Cerasari A, Dominioni I, Sanesi \\ L, Gandolfo V, D'Abbondanza M, Bisogni V, Vaudo \\ G, Pucci G
}

Unit of Internal Medicine, Terni University Hospital Department of Medicine and Surgery, University of Perugia, Perugia, Italy

INTRODUCTION: Atrial fibrillation (AF) is a major risk factor for cardio-embolic stroke. Patients admitted to the Internal Medicine wards often show increased AF prevalence, as the result of acute clinical conditions fostering $\mathrm{AF}$ (e.g. sepsis, tissue hypoxia, electrolytic disturbances). The systematic use of devices enabling AF screening during automated blood pressure (BP) measurement could increase the detection of asymptomatic cases of AF. 
AIM: We evaluated the diagnostic accuracy of the Microlife WatchBP Afib in a cohort of patients admitted to the Internal Medicine ward.

METHODS: 113 patients (mean age $77 \pm 14$ years, men $50 \%$, BP $127 / 71 \pm 18 / 10 \mathrm{mmHg}$ ), consecutively admitted to the Internal Medicine ward of the Terni University Hospital during the period between November 2019 and February 2020, were evaluated. We excluded subjects with pacemaker-induced rhythm, or upper arm circumference $<22 \mathrm{~cm}$ or $>42 \mathrm{~cm}(\mathrm{n}=11)$. In each patient, BP serial measurement (3 measures) was repeated twice. During BP measurement, an ECG was performed.

RESULTS: AF was diagnosed by ECG in 19 patients. In three of them, a new-onset AF was diagnosed. Patients with AF were older ( 86 vs 75 years, $p<0.01$ ), and more frequently had arterial hypotension (systolic $\mathrm{BP}<100$ $\mathrm{mmHg}, 16 \%$ vs $2 \%, \mathrm{p}=0.02$ ). AF screening was positive in 11 out of 19 patients with AF diagnosed by ECG, whereas all patients showing sinus rhythm $(n=83)$ were screened as negative (specificity $100 \%$, sensitivity $58 \%$, Kappa Cohen's 0.70, $\mathrm{p}<0.001$, substantial agreement). Two out the three cases of new-onset AF were correctly identified at the screening by the device. Among individuals with AF diagnosed by ECG, those with negative screening showed a significantly lower coefficient of variability of R-R intervals than subjects screened as positive $(13 \%$ vs $22 \%, \mathrm{p}<0.01)$.

CONCLUSIONS: In patients admitted to the Internal Medicine ward, AF screening through an automated BP measurement device, the Microlife WatchBP Afib, was effective in detecting $\mathrm{AF}$ in a substantial proportion of patients, particularly those with new-onset AF. The suboptimal sensitivity could partly reflect algorithm failure in detecting AF with a relatively regular ventricular rhythm.

\section{UNATTENDED VERSUS ATTENDED BP MEASUREMENT: RELATIONSHIP WITH 24 HOURS BLOOD PRESSURE VALUES}

\author{
M Salvetti, A Paini, F Bertacchini, C Aggiusti, D \\ Stassaldi, S Capellini, G. Saccà, C Arnoldi, C. De \\ Ciuceis, D. Rizzoni, M. Bertini, R. Gatta, ML Muiesan
}

Internal Medicine, University of Brescia, Italy

INTRODUCTION: It has been suggested that measurement of "unattended" or "automated oscillatory" blood pressure (BP) values (AOBP) may provide advantages over conventional BP measurement. More data are needed on the relationship between BP values measured by the conventional approach, by AOBP and values obtained by $24 \mathrm{~h}$ $\mathrm{BP}$ monitoring (ABPM).
METHODS: In 607 consecutive patients attending the outpatient clinic at an ESH Excellence Centre, BP values were measured by the physician with an automated oscillometric device (Omron HEM 9000Ai, mean of 3 measurements), after $5 \mathrm{~min}$ of rest. After the measurement of BP by the physician, the patient was left alone in the room, and the device was programmed to automatically perform 3 $\mathrm{BP}$ measurements after $5 \mathrm{~min}$. ABPM was performed in all subjects.

RESULTS: Mean age was $60 \pm 13$ yrs, 53\% + , BMI $26 \pm 4,77 \%$ with a previous diagnosis of hypertension (58\% treated). Unattended systolic BP (SBP) $(126.4 \pm 14.5)$ was lower as compared to attended SBP $(132.7 \pm 15.9 \mathrm{mmHg})$ and to $24 \mathrm{~h}$ daytime $\mathrm{BP}$ $(129.4 \pm 14.5 \mathrm{mmHg})$. Attended and unattended SBP were similarly correlated to daytime BP $(r=0.649, R=0.669$, $\mathrm{P}<0.000, \mathrm{p}$ for the comparison between $\mathrm{r} n \mathrm{~s})$. The differences $(\Delta)$ between SBP values obtained using the three techniques were $6.3 \pm 7.7 \mathrm{mmHg}$ (attended-unattended), $3.5 \pm 12.2 \mathrm{mmHg}$ (attended-daytime BP) and $-2.8 \pm 11.0$ (unattended-daytime).

CONCLUSIONS: Our findings indicate that "unattended BP" measurement provides values significantly lower as compared to measurements obtained in the presence of the physician and also to $24 \mathrm{~h}$ daytime BP. In our population $\mathrm{BP}$ values obtained by the unattended approach are similar to $24 \mathrm{~h}$ daytime BP values. However $24 \mathrm{~h}$ daytime BP was similarly correlated to both attended and unattended BP.

\section{PROGNOSTIC VALUE OF DAYTIME AND NIGHTTIME BLOOD PRESSURE IN TREATED HYPERTENSIVE PATIENTS ACCORDING TO AGE AND GENDER}

Francesca Coccina ${ }^{1}$, Anna M. Pierdomenico ${ }^{1}$, Jacopo Pizzicannella ${ }^{1}$, Umberto Ianni ${ }^{1}$, Gabriella Bufano ${ }^{1}$, Rosalinda Madonna ${ }^{2}$, Oriana Trubiani ${ }^{1}$, Francesco Cipollone $^{1}$, Sante D. Pierdomenico ${ }^{1}$

${ }^{1}$ Università degli Studi Gabriele d'Annunzio ChietiPescara, Chieti; ${ }^{2}$ Università degli Studi di Pisa, Pisa

AIM: We assessed the prognostic value of daytime and night-time blood pressure (BP) in adult ( $<65$ years) or old ( $>65$ years) women or men with treated hypertension.

METHODS: We studied 2264 sequential treated hypertensive subjects aged 30 to 90 years who were prospectively recruited from December 1992 to December 2012. All the individuals underwent clinical evaluation, electrocardiogram, routine laboratory tests, echocardiographic examination and non-invasive ambulatory BP monitoring. 
RESULTS: During the follow-up (mean 10 years), 523 cardiovascular events occurred. After adjustment for covariates both daytime and night-time BP were always associated with outcomes, that is, hazard ratio (95\% confidence interval per $10 \mathrm{mmHg}$ increment) 1.22 (1.04-1.43) and 1.20 (1.04-1.37), respectively, in adult women, 1.30 (1.18-1.43) and $1.21(1.10-1.33)$, respectively, in adult men, 1.21 (1.10-1.33) and 1.18 (1.07-1.31), respectively, in old women, and 1.16 (1.01-1.33) and 1.28 (1.14-1.44), respectively, in old men. When daytime and night-time BP were further and mutually adjusted, daytime and night-time BP had comparable prognostic value in adult and old women, daytime BP remained associated with outcomes in adult men (hazard ratio $1.40,95 \%$ confidence interval 1.13-1.74 per $10 \mathrm{mmHg}$ increment) and night-time BP remained associated with outcomes in old men (hazard ratio 1.35 , 95\% confidence interval $1.11-1.64$ per 10 $\mathrm{mmHg}$ increment).

CONCLUSIONS: Daytime and night-time BP have similar prognostic impact in adult and old women with treated hypertension, whereas daytime and night-time BP are superior to each other in predicting prognosis in adult and old men, respectively.

\section{ECHOCARDIOGRAPHY-GUIDED CATHETER IMPLANTATION IMPROVES CONSCIOUS RADIOTELEMETRIC BLOOD PRESSURE MEASUREMENT IN MICE}

\section{Carnevale L, Carnevale R, Cifelli G, Mastroiacovo F, Carnevale D, Lembo G}

INTRODUCTION: The study of hypertension in experimental models requires techniques leveraging the implant of radiotelemetric catheters devices. While this technique is the gold standard, it presents some objective difficulties in the complexity of surgery and the scarce catheter positioning precision due to vascular tree variability of the single samples.

AIM: Our approach aims at establishing an ultrasound guided metric to optimize the catheter positioning, ensuring the pressure measurement reliability.

METHODS: Thirty anesthetized mice (all of C57BL6/J strain) underwent surgery to implant the HD-X11 (DSI instruments) pressure catheter device. Radio signals were acquired by the Physiotel RPC-1 receiver and analysis were carried on Ponemah 6.33 acquisition and blood pressure analysis platform. Recording were performed in a dedicated room, with 12-12 h light dark cycle. Catheter positioning was evaluated by aortic arch echo, performed on a Vevo2100 (VisualSonics, Fujifilm), extracting two parameters: -distance from the center of the aortic arch lumen expressed in percentage -angle between catheter tip and the lower wall of aortic arch. All measurements were performed on VevoLab analysis platform (VisualSonincs, Fujifilm).

RESULTS: We established the Bad Data Marks (BDM) extracted from Ponemah platform as quality metric for our blood pressure recordings. We found a linear correlation between the Tip Distance from the Center (TDC) and the BDM $\left(r^{2}=0.14, p=0.039\right)$, with better tracks recordings obtained in the proximity of the center of the aortic arch vessel. Moreover, we found a tendency of correlation between the Tip Angle of Incidence (TAI) with the overall BDM percentage $\left(r^{2}=0.11, p=0.067\right)$. We obtained the best tracks in case of near 0 TAI and TDC, with bad tracks concentrated in regions with more than $10 \%$ of deviation from the center or more than 10 degrees from the optimal direction. CONCLUSIONS: Our work establish a new protocol to optimize the implant of radiotelemetric devices in the small animal. This approach is fundamental to improve the reliability of the measurement and the fidelity of the blood pressure wave across days of registration. Moreover, with our approach we can maximize the number of optimal track to lower the impact of animal usage in respect of the $3 \mathrm{Rs}$ principles.

\section{Nutraceuticals}

EFFECTIVENESS AND SAFETY OF A NOVEL
NUTRACEUTICAL COMPOUND ADDED TO
EZETIMIBE, IN STATIN-INTOLERANT
HYPERCHOLESTEROLEMIC SUBJECTS WITH
MODERATE-TO-HIGH CARDIOVASCULAR RISK

Gioia Torin ${ }^{1}$, Salvatore Lenti ${ }^{2}$, Mariaceleste Nicoletti ${ }^{3}$, Andrea Fratter ${ }^{4}$, Marzia Pellizzato ${ }^{5}$, Alberto Mazza ${ }^{1,2}$

${ }^{1}$ ESH Excellence Hypertension Centre, Internal Medicine Unit, Santa Maria della Misericordia General Hospital, AULSS 5 Polesana, and ${ }^{2}$ Italian Nutraceutical Society (SINut), Triveneto Area, Rovigo, Italy; ${ }^{2}$ Hypertension Centre and Internal Medicine and Geriatrics, San Donato Hospital, USL 8, Arezzo, Italy; ${ }^{3}$ Krea Innovazione srl, Milano, Italy; ${ }^{4}$ President of the Italian Society of Nutraceutical Formulators (SIFNUT); ${ }^{5}$ Vice-President of the Italian Society of Nutraceutical Formulators (SIFNUT)

INTRODUCTION: In the event of statin intolerance, the main guidelines recommend to use bile acid sequestrants, fibrates and ezetimibe (EZE) in monotherapy, but their benefits in improving lipid pattern are quite modest. 
AIM: This study attempted to evaluating the effectiveness and safety of a novel nutraceutical compound (NC) associated with EZE on the modulation of blood lipids profile in statin-intolerant patients with moderate-to-high CV risk. METHODS: Ninety-six statin-intolerant hypertensive and hyper-cholesterolemic subjects treated pharmacologically with EZE $10 \mathrm{mg}$ daily, were randomised in open label $(\mathrm{n}=48)$ to take for 3 months a NC containing MonacolinK $5 \mathrm{mg}$, Berberine Hydrochloride, t-Resveratrol, Quercetin and Chromium. The control group $(\mathrm{n}=48)$, assumed only EZE in monotherapy at the same dosage; both groups followed a standardised lipid-lowering diet. The total serum cholesterol (TC), low density lipoprotein cholesterol (LDLC), high density lipoprotein cholesterol (HDLC), triglycerides (TG), aspartate aminotransferase (AST), alanine aminotransferase (ALT), and creatinine phosphokinase (CPK) levels were compared at the follow-up in both groups using Student's t-test.

RESULTS: TC and LDL levels reduced in both groups, but were lower in the group treated with EZE + NC $(-25.9 \%$ vs $-15 \%, \mathrm{p}<0.05$ and $-38.7 \%$ vs $-21.0 \%, \mathrm{p}<0.05$, respectively). No significant changes were observed in both groups about TG decrease $(-9.4 \%$ vs. $-11.7 \%, \mathrm{NS})$ and HDLC increase $(+4.2 \%$ vs. $+1.1 \%$, NS). The AST, ALT and CPK levels increased in the group treated with the EZE + $\mathrm{NC}$ compared to the control group, but overall remained within the acceptability range. There was no difference concerning the efficacy of lipid-lowering treatment depending on gender and no patients dropped out of the study.

CONCLUSIONS: In the short-term, the EZE + NC combination therapy is well-tolerated and effective in improving TC and LDLC levels in statin-intolerant patients with moderate-to-high cardiovascular risk.

\section{Kidney}

\section{HEART RATE AS A SYMPATHETIC MARKER IN CHRONIC KIDNEY DISEASE}

Gino Seravalle ${ }^{1}$, Fosca Quarti Trevano ${ }^{2}$, Raffaella Dell'oro $^{2}$, Silvio Bertoli ${ }^{2}$, Giuseppe Mancia ${ }^{2}$, Guido Grassi $^{2}$

${ }^{1}$ Cardiologia, Osp San Luca, Istituto Auxologico Italiano, Milano, Italy, ${ }^{2}$ Clinica Medica University Milano Bicocca, Milano, Italy

INTRODUCTION: Chronic kidney disease (CKD) is characterized by a pronounced sympathetic overactivity as documented by the marked increase in muscle sympathetic nerve traffic (MSNA) and in plasma norepinephrine reported in this condition. Whether and to what extent in CKD heart rate (HR) reflects the adrenergic overdrive remains undefined. It is also undefined the relative validity of the different adrenergic markers in reflecting renal dysfunction.

METHODS: In 82 CKD patients, aged $58.4 \pm 1.1$ years (mean \pm SEM), we measured resting clinic blood pressure, HR (EKG), venous plasma norepinephrine (NE, HPLC) and MSNA (microneurography). The same measurements were made in 24 age-matched healthy controls. RESULTS: HR was significantly greater in CKD than in controls $(74.0 \pm 1.1$ versus $68.2 \pm 1.8$ beats/minute, $\mathrm{P}<0.02)$ and significantly directly related to the elevated plasma NE and MSNA values $(r=0.22$ and $r=0.39$, $\mathrm{P}<0.05$ and $\mathrm{P}<0.0003$, respectively). Both MSNA and NE were significantly and inversely related to the estimated glomerular filtration rate. The correlation did not achieve statistical significance for HR. Similar results were obtained examining the relationships with left ventricular mass index.

CONCLUSIONS: Our data show that in CKD 1) not only peripheral but also cardiac sympathetic drive is markedly enhanced and 2) HR can be regarded as a marker of the adrenergic overdrive characterizing this condition. The reliability of HR as sympathetic marker appears to be limited, however, this variable being unable to closely reflect, at variance from MSNA and NE, the interindividual differences in renal dysfunction and the accompanying structural cardiovascular alterations.

\section{SYMPATHETIC NERVE TRAFFIC OVERACTIVITY IN CHRONIC KIDNEY DISEASE AND ITS CORRELATES: A SYSTEMATIC REVIEW AND META-ANALYSIS}

Grassi $G^{1}$,Biffi $A^{2,}$,Seravalle $G^{3}$,Bertoli $S^{4}$,Airoldi $\mathrm{F}^{4}$, Corrao $\mathrm{G}^{2}$,Pisano $\mathrm{A}^{5}$,Mallamaci $\mathrm{F}^{5}$, Mancia $\mathrm{G}^{1}$,Zoccali $\mathrm{C}^{5}$

${ }^{1}$ Clinica Medica, Department of Medicine and Surgery, University Milano-Bicocca, ${ }^{2}$ Department of Statistics and Quantitative Methods, University Milano-Bicocca; ${ }^{3}$ Cardiologia, Osp. S. Luca IRCCS Istituto Auxologico Italiano, Milano; ${ }^{4}$ IRCCS Multimedica, Sesto San Giovanni, Milan and ${ }^{5}$ Consiglio Nazionale delle RicercheIstituto di Fisiologia Clinica, Clinical Epidemiology and Physiopathology of Renal Diseases and Hypertension, Reggio Calabria, Italy

INTRODUCTION: Microneurographic recording of sympathetic nerve traffic (MSNA) have shown that sympathetic activation may occur in chronic kidney disease 
(CKD). However, the small sample size of the available studies, presence of comorbidities, heterogeneity of the patients examined represented major weaknesses not allowing to draw definite conclusions, particularly for early phases of CKD.

METHODS: The present meta-analysis evaluated 1020 subjects recruited in 29 microneurographic studies, assessing MSNA quantification in different CKD stages, excluding comorbidities. Assessment was also extended to the relationships of MSNA with other neuroadrenergic markers, such as plasma norepinephrine and heart rate, anthropometric variables, and markers of renal function.

RESULTS: CKD patients stratified by stage coherently showed higher average MSNA values, about $22 \%$ in stage $1-2$ and $80 \%$ in hemodyalisis $(\mathrm{P}<0.001)$ as compared to controls. MSNA when expressed as bursts/min was significantly related to plasma creatinine in CKD patients $(\mathrm{r}=0.70, \mathrm{P}<0.01)$. When combined data of patients and controls were analyzed across the various glomerular filtration rate classes MSNA values were progressively higher across classes denoting renal dysfunction of increasing severity reaching a maximum in hemodialysis patients ( $\mathrm{P}$ value for trend $<0.01$ ). In the $\mathrm{CKD}$ population MSNA was significantly and directly related to age $(\mathrm{r}=0.57, \beta=0.74, \mathrm{P}=0.02)$, body mass index $(\mathrm{r}=0.75$, $\beta=3.17, P=0.001$ ), but not to albuminuria. No significant correlation was found between MSNA, heart rate, and norepinephrine.

CONCLUSIONS: Thus MSNA is markedly increased in CKD and this increase is (1) independent on comorbidities, (2) not restricted to ESKD patients maintained on chronic dialysis but clearly detectable in mild and moderate, (3) related to the severity of renal dysfunction being an early phenomenon which worsens gradually when the degree of renal dysfunction worsens.

\section{INCREASED SERUM URIC ACID LEVELS ARE ASSOCIATED TO RENAL ARTERIOLOPATHY AND PREDICT POOR OUTCOME IN IgA NEPHROPATHY}

\section{Elisa Russo ${ }^{1}$, Stefania Drovandi ${ }^{1}$, Gennaro Salvidio ${ }^{1}$, Daniela Verzola ${ }^{1}$, Pasquale Esposito ${ }^{1}$, Giacomo Garibotto $^{1}$, Francesca Viazzi ${ }^{1}$ \\ ${ }^{1}$ Ospedale Policlinico San Martino, Department of Internal Medicine, Genoa, Italy}

INTRODUCTION: $\operatorname{Ig}$ A nephropathy $(\operatorname{IgAN})$ is the most common primary glomerulonephritis worldwide and a leading cause of End-Stage Renal Disease (ESRD). In addition to classical progression factors, other atherosclerosis-related factors, including hyperuricemia (HU), have been associated to the renal progression of IgAN. Increased serum uric acid (SUA) levels are well known to be concomitant of cardiovascular and kidney diseases, and have been proposed to be implicated in the development of arteriolar damage (AD).

AIM: to explore the correlation between SUA levels, renal damage and its implication for outcome in $\operatorname{IgAN}$ patients. METHODS: Clinical, laboratory and histologic data of 145 patients with biopsy proven IgAN were collected and retrospectively analyzed to determine the correlation between SUA levels, renal damage and the primary outcome (death or ESRD). Biopsy-proven AD was defined by the presence of arteriolar hyalinosis and/or intimal thickening.

RESULTS: HU, defined as the highest SUA genderspecific tertile, was $>7.7 \mathrm{mg} / \mathrm{dl}$ for males and $>6.2 \mathrm{mg} /$ $\mathrm{dl}$ for females. The prevalence of AD increased with the increase in the SUA level tertiles $(p=0.02)$. At logistic regression analysis SUA was independently related to the presence of $\mathrm{AD}$ (OR 1.75 [95\% CI 1.10-2.93], $\mathrm{p}=0.03$, Table 1). HU and AD had a synergic impact on progression of IgAN. Patients having both $\mathrm{AD}$ and $\mathrm{HU}$, showed a reduced survival free from the primary outcome as compared to those having only one risk factor or neither $(\mathrm{p}=0.01)$.

CONCLUSIONS: SUA levels are independently associated with $\mathrm{AD}$ and poor prognosis in patients with $\mathrm{IgAN}$.

\section{RENAL HEMODYNAMICS AND 10-YEARS RISK OF CARDIOVASCULAR DISEASE PREDICTED BY FRAMINGHAM RISK MODELS AND POOLED COHORT EQUATIONS: AN OBSERVATIONAL STUDY IN HYPERTENSIVE SUBJECTS WITHOUT CARDIOVASCULAR DISEASE}

Giulio Geraci ${ }^{1}$, MD; Marta Maria Zammuto ${ }^{1}$, MD; Nicola Sinatra ${ }^{1}$, MD; Ettore Mancia ${ }^{1}$, MD; Alessandra Sorce $^{1}$, MD; MD; Alessandro Mattina ${ }^{2}$, MD; Emilio Nardi $^{2}$, MD; MD; Santina Cottone ${ }^{1}$, MD; Giuseppe Mulè $^{1}, \mathrm{MD}$

${ }^{1}$ Department of Health Promotion, Mother and Child Care, Internal Medicine and Medical Specialties (PROMISE). Unit of Nephrology and Hypertension. European Society of Hypertension Excellence Center, University of Palermo, Italy; ${ }^{2}$ Department of Health Promotion, Mother and Child Care, Internal Medicine and Medical Specialties (PROMISE).Unit of Internal Medicine University of Palermo, Italy 
INTRODUCTION: Renal resistive index (RRI) has widely proved to be related with subclinical vascular damage in subjects with essential hypertension as well as in other populations. However, few data exist about the association between RRI and cardiovascular events in hypertensive subjects, and it is not known if 10-years risk of cardiovascular disease predicted by validated score equations is associated with impaired intrarenal hemodynamics.

METHODS: A total of 742 subjects with essential hypertension (40-75 years) were enrolled. In all patients, renal resistive index was assessed through Duplex-Doppler ultrasonography and 10-years risk of cardiovascular disease was calculated using both Framingham risk score (FS) and Atherosclerotic CardioVascular Disease Risk Score (ASCVD) through validated equations.

RESULTS: Higher RRI values were observed in patients with calculated cardiovascular risk $\geq 20 \%$ than in those with lower risk (all p $<0.001$ ). RRI was closely associated with both FS and ASCVD in overall study population (all $\mathrm{p}<0.001$ ), with no significantly differences between groups with glomerular filtration rate $\geq$ or $<60 \mathrm{ml} / \mathrm{min}$. At multivariate analyses, these associations held also after correction for traditional factors included in the FS and ASCVD equations (respectively $\mathrm{p}=0.007$ and $\mathrm{p}=0.047$ ). When receiver-operating characteristic curves were built to detect a 10 -years risk of cardiovascular disease $\geq 20 \%$, RRI values $>0.67$ and $>0.65$ were associated with high cardiovascular risk respectively calculated through FS and ASCVD equations.

CONCLUSIONS: Renal resistive index can be considered as a marker of overall cardiovascular risk in hypertensive patients, regardless of renal function.

\section{Therapy, Pharmacoeconomy and Pharmacosurveillance}

\section{AMBULATORY BLOOD PRESSURE CONTROL IN PATIENTS ON TRIPLE ANTI-HYPERTENSIVE THERAPY WITH ACE-INHIBITORS OR ANGIOTENSIN-RECEPTOR-BLOCKERS: ROLE OF TRIPLE FIXED-DOSE COMBINATION AND TREATMENT ADHERENCE}

\author{
Francesco Spannella ${ }^{1,2}$, Federico Giulietti ${ }^{1,2}$, Andrea \\ Filipponi $^{1}$, Sonia Marziali ${ }^{1}$, Riccardo Sarzani ${ }^{1,2}$ \\ 1) Department of Clinical and Molecular Sciences, \\ University "Politecnica delle Marche", Via Tronto 10/a, \\ Ancona, Italy; 2) Internal Medicine and Geriatrics, IRCCS \\ INRCA, Via della Montagnola 81, Ancona, Italy
}

INTRODUCTION: Anti-hypertensive drug combinations containing ACE-inhibitors (ACEi) or angiotensin-receptorblockers (ARB) play a key role in obtaining blood pressure (BP) control, as stated by the 2018 ESC/ESH Guidelines on arterial hypertension.

AIM: to compare the ambulatory BP control rates in hypertensive patients taking triple anti-hypertensive regimens [renin-angiotensin-system inhibitor + calciumchannel-blocker + thiazide/thiazide-like diuretic] containing an ACEi versus an ARB.

METHODS: Observational study on 521 consecutive hypertensives evaluated with $24 \mathrm{~h}$ ambulatory BP monitoring in stable treatment for at least 3 months with a triple anti-hypertensive regimen. An anti-hypertensive treatment intensity (ATI) has been calculated to compare both the different drugs and dosages.

RESULTS: Mean age: $62.7 \pm 12.1$ years; Males: $61.7 \%$. Patients taking ACEi: 190 (36\%). Patients taking ARB: 331 (64\%). No significant differences in mean age, sex, bodymass-index (BMI), smoking, diabetes mellitus, dyslipidemia, estimated-glomerular-filtration-rate (eGFR) were found between the two groups. Patients taking ARB had a significantly slightly higher ATI compared to patients taking ACEi $(5.5 \pm 0.4$ vs $5.4 \pm 0.4, \mathrm{p}=0.017)$. Patients taking ACEi had both higher $24 \mathrm{~h}$-BP control rates (54.8\% vs $44.0 \%$; OR: $1.8 ; \mathrm{p}=0.039)$ and higher daytime BP control rates $(61.8 \%$ vs $49.2 \%$; OR: $1.9 ; \mathrm{p}=0.024)$ than patients taking $\mathrm{ARB}$, even after adjusting for age, sex, BMI, smoking, diabete mellitus, eGFR and ATI. Patients on ACEi-based triple therapy took fewer pills compared to patients on ARB-based triple therapy (22.2\% of patients took a triple ACEi-based fixed-dose combination). After adding this variable in the statistic model, the association between BP control and therapy with ACEi or ARB lost significance.

CONCLUSIONS: We found that an ACEi-based triple therapy is associated with a better ambulatory BP control compared to an ARB-based triple therapy. However, this finding is likely due to the lower number of pills taken by patients, linked to a better adherence to the anti-hypertensive therapy.

\section{PREVALENCE AND PREDICTORS OF ADVERSE DRUG REACTIONS IN OLDER HYPERTENSIVE ADULTS}

Giada Turrin ${ }^{1}$, Antonio Coscarelli, Maria Flora D'Andria, Virginia Tortu', Evelina Giuliani, Andrea Ungar, Giulia Rivasi

${ }^{1}$ Referral Centre for Hypertension Management in Older Adults, Division of Geriatric and Intensive Care Medicine, Careggi Hospital and University of Florence, Italy 
INTRODUCTION: Polipharmacy is common in older people, thus increasing the risk of adverse drufg reactrions. AIM: This study aimed to analyse prevalence and predictors of the most relevant adverse drug reactions in older hypertensive adults.

METHODS: We retrospectively analysed data on antihypertensive- and statin-related adverse drug reactions in adults aged 65 or older evaluated at the Hypertension Clinic of Careggi Hospital, Florance, Italy, between January 2018 and December 2019. Predictors were investigated using multivariate logistic regression.

RESULTS: Of 262 participants (mean age 75.9 years, $55.3 \%$ female), the $19 \%$ of the patients taking ACE-inhibitors reported cough while $33.6 \%$ of the patients taking calcium channel blockers $(\mathrm{CCB})$ reported peripheral edema, occuring more frequently of amlodipine. The prevalence of statin-related myalgia was $23.5 \%$. At multivariate analysis SNRI antidepressants and gastroesophageal reflux disease (GERD) were predictive of ACE-inibitors induced cough (OR 16.220, 95\% CI 1.243-211.621; OR 4.207, 95\% CI 1.249-4.171, respectively) and angiotensin-receptor antagonists were associated with an increased risk of peripheral edema on CCB (OR 2.500, 95\% CI 1.095-5.710). GERD was predictive for statin-induced myalgia (OR 6.737, 95\% CI 1.530-29.662).

CONCLUSIONS: ACE inhibitor-induced cough, CCBrelated peripheral edema and statin-induced myalgia are common in older hypertensive adults. SNRI antidepressants and GERD are independently associated with ACE inhibitor-induced cough, while angiotensin-receptor antagonists are predictive of CCB-related peripheral edema. GERD is also associated with statin myalgia.

\section{Vessels and Endothelium}

\section{ASSOCIATION BETWEEN URIC ACID AND PULSE WAVE VELOCITY IN HYPERTENSIVE PATIENTS AND GENERAL POPULATION: A SYSTEMATIC REVIEW AND META-ANALYSIS}

\author{
Alessandro Maloberti ${ }^{1}$, Paola Rebora ${ }^{2}$, Anita \\ Andreano $^{2}$, Nicola Triglione ${ }^{1}$, Enrico Piccinelli ${ }^{1}$, \\ Matteo Palazzini ${ }^{1}$, Lucia Occhi ${ }^{1}$, Jinwei Sun ${ }^{1}$, \\ Benedetta De Chiara ${ }^{1}$, Guido Grassi ${ }^{1}$, Maria Grazia \\ Valsecchi $^{2}$, Cristina Giannattasio ${ }^{1,3}$ \\ 1- School of Medicine and Surgery, University of Milano- \\ Bicocca, Milan, Italy; 2 - Center of Biostatistics \\ for Clinical Epidemiology, School of Medicine \\ and Surgery, University of Milano-Bicocca, Milan, Italy; 3 \\ - Cardiology IV, "A.De Gasperis" Department, ASTT \\ GOM Niguarda Ca' Granda, Milan, Italy
}

INTRODUCTION: The association between Serum Uric Acid (SUA) and Pulse Wave Velocity (PWV), has been extensively evaluated but with some discrepancies in results. A further limitation refers to the fact that only few data were analyzed taking into account the possible effects of gender.

AIM: to estimate the association between SUA and arterial stiffness in general population and hypertensive patients, as a whole population and as divided by gender, by pooling results from existing studies.

METHODS: Carotid-femoral and brachial-ankle PWV (cf- and ba-PWV) have been analysed separately and subgroup analyses by gender are reported.

RESULTS: Among 692 potentially relevant works, 24 paper were analysed. 7 studies referred to cf-PWV in the general population with an overall positive association at adjusted analysis for both males and females $(0.07-95 \%$ CI: $0.03 ; 0.11$ and $0.06-95 \%$ CI: $0.03 ; 0.09$, respectively). 12 studies referred to ba-PWV in the general population with the finding of a positive association at adjusted analysis for females $(0.04-95 \% \mathrm{CI}: 0.01 ; 0.07)$, but not for males $(0.13-95 \%$ CI $-0.09 ; 0.34)$. In hypertensive patients only 4 studies evaluated cf-PWV and 1 ba-PWV with only one study (with cf-PWV) finding positive association.

CONCLUSIONS: The association between SUA and cfPWV resulted significant in general population in both males and females while it was only significant for female regarding ba-PWV. Furthermore, the few available studies found no significant relationship between SUA and both cfand ba-PWV in hypertensive subjects.

\section{EFFECTS OF INTENSIVE URATE LOWERING THERAPY WITH FEBUXOSTAT IN COMPARISON WITH ALLOPURINOL ON PULSE WAVE VELOCITY IN PATIENTS WITH GOUT AND INCREASED CARDIOVASCULAR RISK: THE FORWARD STUDY}

Giovambattista Desideri ${ }^{1}$, Claudio Borghi ${ }^{2}$

${ }^{1}$ Department Of Life, Health And Environmental Sciences, University Of L'aquila, Italy; ${ }^{2}$ Department Of Medical And Surgical Sciences, Ospedale Malpighi, University of Bologna, Italy

INTRODUCTION: hyperuricemia and gout are strongly related with traditional cardiovascular risk factors and vascular damage.

AIM: to assess whether febuxostat is better than allopurinol in ameliorating pulse wave velocity in patients with hyperuricemia and gout. 
METHODS: a multi-centre, multinational, phase iv, randomized, parallel-group, active-controlled, open label trial with blind end-points evaluation. One hundred and ninetyseven adults with hyperuricemia $(>8 \mathrm{mg} / \mathrm{dl})$ and gout were randomised to febuxostat or allopurinol in a 1:1 ratio for 36 weeks. The primary outcome was the comparison of the effects of febuxostat and allopurinol on changes in pulse wave velocity.

RESULTS: the mean pulse wave velocity values at randomisation and week 36 were respectively $8.69 \mathrm{~m} / \mathrm{s}$ and 9.00 $\mathrm{m} / \mathrm{s}$ for subjects randomised to febuxostat and $9.02 \mathrm{~m} / \mathrm{s}$ and $9.05 \mathrm{~m} / \mathrm{s}$ for subjects randomised to allopurinol. No statistically significant changes in pulse wave analysis by treatment assignment were observed at any time point for any of the assessed parameters. More subjects who received febuxostat had serum urate concentrations $\leq 6 \mathrm{mg} / \mathrm{dl}$ following treatment $(78.3 \%$ vs $61.1 \%$ at week $36, p=0.0137)$. Treatmentemergent adverse events were reported by $51(52.0 \%)$ subjects randomised to febuxostat and 63 (62.5\%) subjects randomised to allopurinol. the majority of events were mild in both treatment groups and included gout flares and arthralgia.

CONCLUSIONS: in patients with hyperuricemia and gout the arterial stiffness remained stable both with febuxostat and allopurinol. Febuxostat was more effective and faster than allopurinol in achieving the serum uric acid target. Both treatments were safe and well tolerated.

\section{THE RELATIONSHIP BETWEEN TELOMERE LENGTH AND MARKERS OF VASCULAR AGEING: A SYSTEMATIC REVIEW AND META- ANALYSIS}

\author{
Martina Chiriacò ${ }^{1}$, Marinos Kosmopoulos ${ }^{2}$, Nicola \\ Riccardo Pugliese ${ }^{1}$, Agostino Virdis ${ }^{1}$, Stefano Taddei ${ }^{1}$, \\ Georgios Georgiopoulos ${ }^{2}$, Stefano Masi $^{1}$

\section{${ }^{1}$ Department of Clinical and Experimental Medicine, University of Pisa, Italy; 2 Department of Clinical Therapeutics, National and Kapodistrian University of Athens, Greece}

INTRODUCTION: Pulse Wave Velocity (PWV) and carotid Intima-Media Thickness (cIMT) have been used as markers of vascular ageing. To be considered reliable markers it is necessary that they reflect the process of biological aging, which is well represented by Leukocyte Telomere Length (LTL).

AIM: This meta-analysis explores the association between PWV and cIMT and LTL to evaluate their role as markers of vascular ageing.

METHODS: the main scientific databases (PubMed, Medline, Embase, and Cinahl) were searched and correlation and regression coefficients between LTL and cIMT or PWV were retrieved from the papers. Collected data was analysed using a random-effect meta-analysis model.

RESULTS: 21 studies were selected for a total of 10306 patients. A reverse association between LTL and cIMT was found (pooled $\mathrm{r}=-0.249,95 \%$ CI $-0.37 /-0.128$, $\mathrm{P}<0.001$ ), and between LTL and PWV (pooled $\mathrm{r}=$ 0.194, 95\% CI $-0.290 /-0.100, \mathrm{P}<0.001$ ) (Figure 6).

CONCLUSIONS: PWV and cIMT are inversely associated with LTL and therefore can be considered reliable markers of vascular ageing.

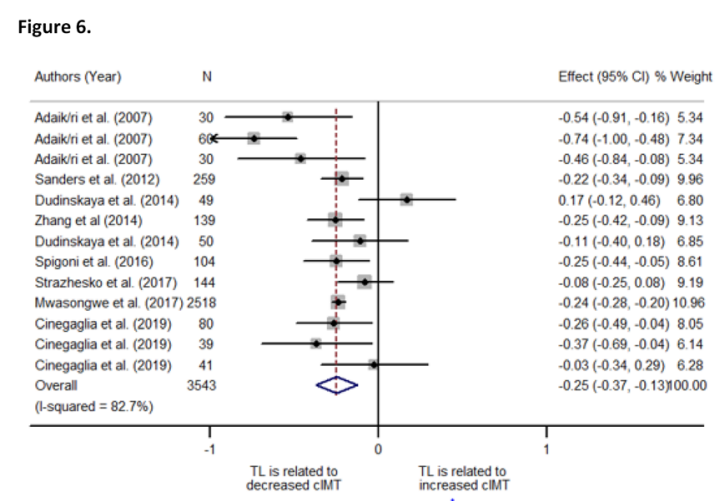

NOTE: Weights are from random-effects mode

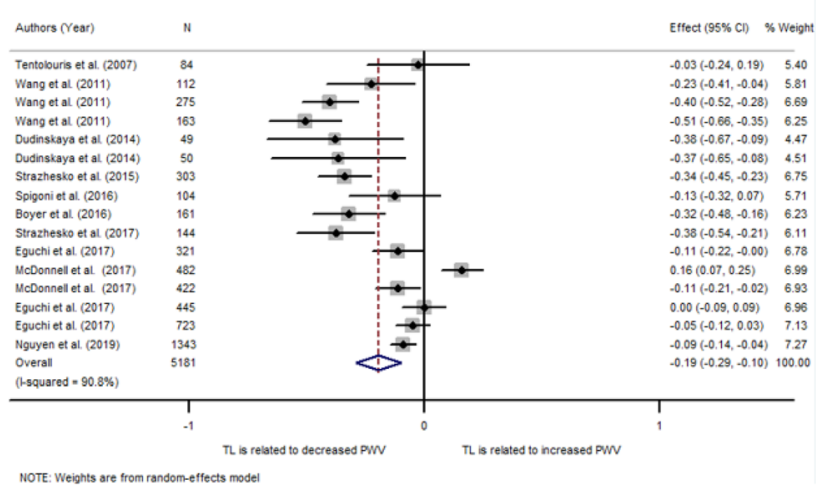

\section{GENERATION AND CHARACTERIZATION OF A TRANSGENIC MOUSE MODEL WITH SELECTIVE DELETION OF THE TYROSINE HYDROXYLASE GENE IN THE ENDOTHELIUM}

Antonella Fiordelisi ${ }^{1}$, Jessica Gambardella ${ }^{1}$, Michele Ciccarelli $^{2}$, Guido Iaccarino ${ }^{1}$, Daniela Sorriento ${ }^{1}$

${ }^{I}$ Dipartimento di Scienze Biomediche Avanzate, Università Federico II di Napoli; ${ }^{2}$ Dipartimento di Medicina, 
Chirurgia e Odontoiatria "Scuola Medica Salernitana"/ DIPMED, Università degli Studi di Salerno

INTRODUCTION: We recently showed that the endothelium can synthesize and release catecholamines in response to hypoxia. Catecholamines play a key role in regulating vascular tone especially in response to vascular damage, therefore the ability of endothelial cells to produce them autonomously could represent a self-defense mechanism to protect cells from vascular damage and ischemia. It is therefore essential to clarify the pathophysiological function of endothelial catecholamine production and its effect in response to vascular damage. To date, no mouse model allows to achieve this goal, therefore the aim of the study is the generation and characterization of a transgenic mouse model in which the tyrosine hydroxylase $(\mathrm{TH})$ gene, the rate-limiting enzyme in the synthesis of catecholamines, is selectively deleted in the endothelium by the CRELOX system with specific tissue expression of the CRE recombinase.

METHODS: we crossed TH-FLOX mice and Tie2-CRE mice to obtain mice with endothelial knock-out of the TH gene in heterozygosity $(\mathrm{TH} \pm)$ and the latter were crossed with each other to obtain the knock-out of the TH gene in homozygosity (TH-/-).

RESULTS: The first interesting finding of the study derives from the results of these crossings. In fact, in all the crossings between $\mathrm{TH} \pm$ we have never obtained pups with the $\mathrm{TH}-/-$ genotype suggesting that the total $\mathrm{TH}$ deletion in the endothelium could be lethal and that the endothelial production of catecholamines would play an important role in embryonic development. We then evaluated the production of total catecholamines in blood samples of $\mathrm{TH} \pm$ mice compared to the relative controls by ELISA and showed a significant reduction (Norepinephrine: $-22 \pm 6.4 \%$; Epinephrine: $-56 \pm 3.1 \%$, $\mathrm{p}<0.05)$.

CONCLUSIONS: $\mathrm{TH} \pm$ mice represent a useful model for the study of endothelial catecholamines. Also, the data obtained so far indicate that the catecholamines produced by the endothelium represent a significant rate and that they play a key role in embryonic development.

\section{ORTHOSTATIC HYPOTENSION, ARTERIAL STIFFNESS AND AGING}

Bortolazzi E, Macchi F, Amadio G, Franconi A., Trentin M, Rossi A, Zamboni M and Fantin F

University of Verona, Verona, Italy
INTRODUCTION: Orthostatic hypotension $(\mathrm{OH})$ is an independent risk factor for cardiovascular morbidity and mortality. Arterial stiffness, typical of cardiovascular aging, has been shown to be a pathophysiological mechanism linking $\mathrm{OH}$ and increased $\mathrm{CV}$ risk.

AIM: to evaluate the relationship between $\mathrm{OH}$ and arterial stiffness and SubEndocardial Viability Ratio (SEVR) and moreover to identify the main predictors of orthostatic hypotension, Carotid-Femoral Pulse Wave Velocity (PWVcf) and SEVR.

METHODS: Eighty-five patients were enrolled (average age $83.45 \pm 6.38$ ) in Verona's AOUI Geriatric ward. They underwent blood pressure, heart rate, body weight measurements. In addition, comorbidity, arterial stiffness (PWVcf measured by applanation tonometry), SEVR and biochemical indexes (haemoglobin, creatinine, electrolytes, lipids).

RESULTS: Prevalence of orthostatic hypotension was $46.6 \%$. The SEVR values corrected for arterial oxygen and hemoglobin content were statistically lower in $\mathrm{OH}$ patients $(\mathrm{p}=0.039)$; PWVcf values were statistically higher in $\mathrm{OH}$ subjects $(\mathrm{p}=0.041)$. In a binary logistic regression, PWVcf was the only significant predictor of $\mathrm{OH}$ (OR $1.123 ; \mathrm{p}=0.039 ; \mathrm{IC}=1.006-1.17)$. In a backward logistic regression model gender, creatinine clearance and $\mathrm{OH}$ were significant predictors of SEVR corrected for O2 content. Mean arterial pressure, creatinine clearance and $\mathrm{OH}$ were significant predictors of PWV.

CONCLUSIONS: In conclusion, the data collected within this study show that $\mathrm{OH}$ is related to increased arterial stiffness, justifying its higher prevalence rate in elderly patients. $\mathrm{OH}$ was also associated with reduced values of corrected SEVR, suggesting a possible role of reduced subendocardial vascularization in rising cardiovascular morbidity and mortality in patient with $\mathrm{OH}$.

\section{REDUCED EXPRESSION OF THE G PROTEIN- COUPLED RECEPTOR KINASE 2 (GRK2) IN THE ENDOTHELIUM ACCELERATES THE PROCESS OF AORTIC VALVE CALCIFICATION}

\author{
MR Rusciano ${ }^{1,2}$, D Sorriento ${ }^{3}$, V Valerio ${ }^{4}$, \\ G. Iaccarino ${ }^{3}$, P. Poggio ${ }^{4}$, M. Ciccarelli ${ }^{1}$ \\ ${ }^{1}$ University of Salerno, Salerno, Italy; ${ }^{2}$ Montevergine \\ Cardiology Hospital, Mercogliano, Italy; ${ }^{3}$ Federico II, \\ University of Naples Dept of Advanced Biomedical \\ Sciences; ${ }^{4}$ Centro Cardiologico Monzino, Milan, Italy
}

INTRODUCTION: GRK2 is a pivotal regulator of cardiovascular functions. We previously demonstrated that the lack of this protein in the endothelium promotes vascular 
inflammation and atherosclerosis in mice due to increased mitochondrial reactive oxygen species (ROS). We, therefore, hypothesized that GRK2 could be involved in the process of aortic valve calcification (AVC).

AIM: To evaluate the role of GRK2 in AVC implementing in vivo and in vitro models.

METHODS: We used 12 months old mice with selective endothelial knock-out of GRK2 (Tie2CRE-GRK2fl/fl) in EC compared to control (GRK2fl/fl) to evaluate the presence of microcalcification in the aortic valve leaflets by histological analysis. We also employed isolated human valve endothelial cells (VEC) from control and calcified (CAVS) aortic valve leaflets to assess GRK2 expression and subcellular compartmentalization.

RESULTS: As previously demonstrated, GRK2 removal from EC induces early atherosclerotic lesions. Here, we observed that GRK2fl/fl mice of 12 months-old display presence of microcalcification, as expected. However, this phenotype is significantly more pronounced in the Tie2CRE-GRK2fl/fl, demonstrating that the lack of GRK2 in the EC accelerates the calcific degeneration of the aortic valve in mice (Figure 7). In vitro, we observed a significant downregulation of GRK2 expression into the mitochondria of CAVS VECs than control VECs (Figure 8A), which associates to an increased ROS production. A previous report demonstrated that BARKct transfection in macrophage increases mitochondrial biogenesis and reduces ROS production. Here, using the cloning strategy depicted in Figure $8 \mathrm{~B}$, we cloned several small sequences of the $\mathrm{PH}$ domain of GRK2 into the pcDNA3.1 plasmid, named as PH\#1-4. We found that the transfection into HEK293 of the PH\#3 potently increased GRK2 localization into the mitochondria as compared to BARKct, PH4, and pcDNA3.1 as control (Figure 8C). PH3 also determined increased biogenesis (Figure 8D) and reduced ROS production stimulation (Figure 8E). These data support the concept that a smaller portion of the PH domain of BARKct can reproduce its biological effect.

CONCLUSIONS: Our data suggest a direct involvement and a casual role of GRK2 expression/localization in the pathogenesis of CAVS. The GRK2 re-localization into mitochondria is a potential novel strategy to counteract ROS production and the process of AVC.

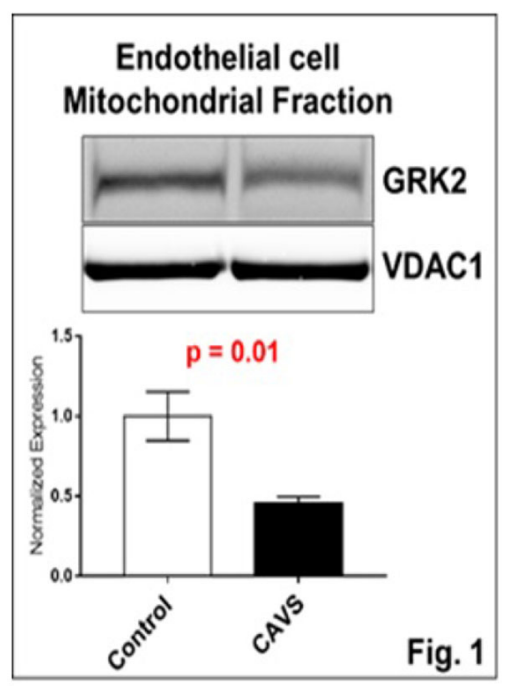

Figura 1 Local izzazione mitocondriale di GRK2 nelle celle endoteliali di pazienti con calcificazione della
valvola

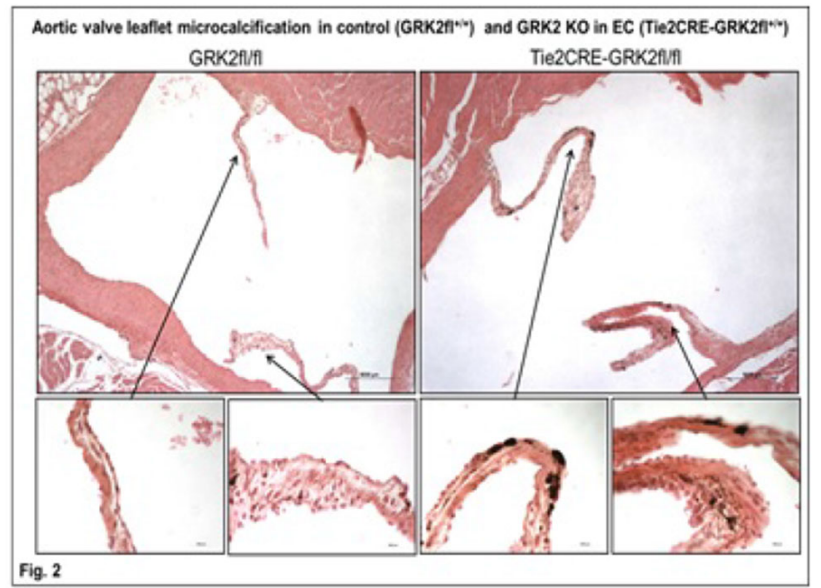

Figura 2 valutazione della presenza di GRK2 nelle microcalcificazioni della valvola aortica in modelli animali 


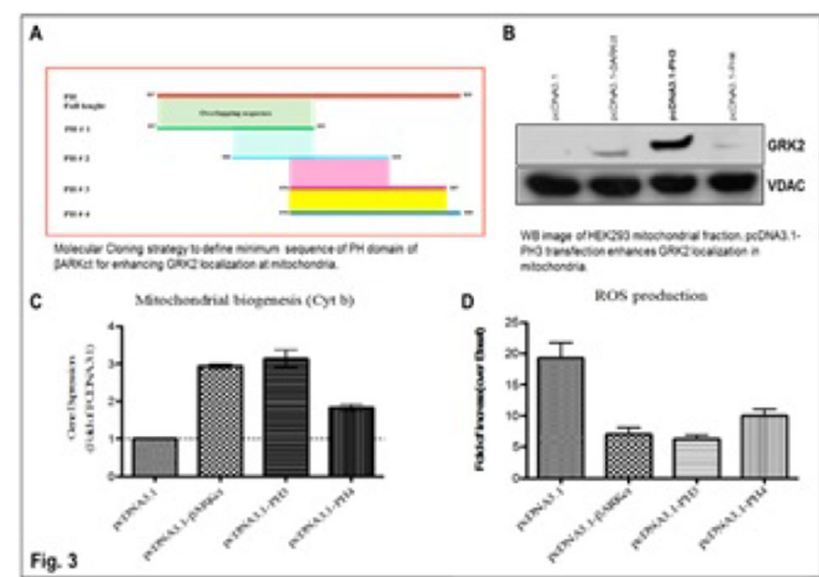

Figura 3 La localizzazione mitocondriale di GRK2 riduce la produzione di ROS

\section{STRUCTURAL ALTERATIONS OF MICROCIRCULATION IN CANCER PATIENTS TREATED WITH ANTIANGIOGENIC DRUGS}

Valeria Brami ${ }^{1}$, Maria Antonietta Coschignano ${ }^{1}$, Carolina De Ciuceis ${ }^{1}$, Claudia Rossini ${ }^{1}$, Alfredo Berruti ${ }^{2}$, Claudia Agabiti Rosei ${ }^{1}$, Anna Paini ${ }^{1}$, Deborah Cosentini ${ }^{2}$, Alberto Dalla Volta ${ }^{2}$, Massimo Salvetti ${ }^{1}$, Enzo Porteri ${ }^{1}$, Alina Petelca ${ }^{1}$, Matteo Nardin ${ }^{1}$, Giulia Chiarini $^{1}$, Maria Lorenza Muiesan ${ }^{1}$, Damiano Rizzoni $^{1,3}$

${ }^{1}$ Department of Clinical and Experimental Sciences, University of Brescia, ${ }^{2}$ Department of Clinical and Experimental Sciences, University of Brescia, ${ }^{3}$ Division of Medicine, ASST Spedali Civili- Montichiari, University of Brescia

INTRODUCTION: Development of hypertension as well as alterations in microcirculation (decreased capillary density) have been recently reported to be associated with tyrosine kinase inhibitor (TKI) treatment in cancer patients (Dalbeni A, et al. Cancers 2019). An increased blood pressure has also been demonstrated with direct anti-VEGF inhibitor.

AIM: to evaluate whether TKI and direct anti-VEGF agents may affect also the structure of retinal arterioles which seems to have prognostic significance in term of cardiovascular events.

METHODS: For this purpose, we included 14 patients with a diagnosis of cancer (renal $\mathrm{n}=8$, lung $\mathrm{n}=2$, gastrointestinal $\mathrm{n}=2$, tyroid $\mathrm{n}=1$, breast $\mathrm{n}=1$ ) underwent a treatment neither with a TKI $(\mathrm{n}=11$ : sunitimib $\mathrm{n}=5$, pazopanib $n=4$, nintedanib $n=1$, lenvatinib $n=1$ ) or with an anti-VEGF antibody $(n=3$ : bevacizumab $n=2$, ramucirumab $\mathrm{n}=1$ ). All patients were submitted to ambulatory monitoring blood pressure for blood pressure evaluation. Basal and total (after venous congestion) capillary density were assessed by capillaroscopy whereas retinal arteriole morphology was obtained by Adaptive Optic in order to evaluate microcirculation. Patients were evaluated before starting the antiangiogenic therapy (T0) and re-evaluated after three (T3) and six (T6) months after treatment. Changes in antihypertensive treatment was also assessed.

RESULTS: Result are reported in the Table. Systolic and diastolic blood pressure values were similar in all patients at T3 and T6 compared to T0. However, during the study antihypertensive treatment was optimized (increased dose and/or added additional agents) in $57 \%$ of patients $(n=8)$. No differences were observed in retinal arteriole structural parameters. Basal capillary density resulted reduced by antiangiogenic drugs after three or six months (Table).

CONCLUSIONS: Our preliminary data suggest that an increase of antihypertensive treatment is necessary in patients treated with a tyrosine kinase inhibitor (TKI) or a direct VEGF inhibitor, confirming a pro-hypertensive effects of these drugs. However, under adequate blood pressure control, a worsening of basal capillary density but no changes in retinal arteriole morphology might be observed.

Table.
\begin{tabular}{|l|c|c|c|}
\hline \multicolumn{1}{|c|}{ Parameter } & T0 & T3 & T6 \\
\hline 24hour systolic blood pressure (mm Hg) & $122.6 \pm 14.7$ & $123.3 \pm 12.2$ & $124.8 \pm 24.4$ \\
\hline 24hour diastolic blood pressure $(\mathrm{mm} \mathrm{Hg})$ & $71.7 \pm 10.2$ & $70.9 \pm 8.0$ & $70.7 \pm 8.9$ \\
\hline Lumen, $\mu \mathrm{m}$ & $86.42 \pm 17.00$ & $87.14 \pm 11.4$ & $89.25 \pm 11.71$ \\
\hline External diameter, $\mu \mathrm{m}$ & $111.5 \pm 21.6$ & $111.9 \pm 14.1$ & $114.4 \pm 14.7$ \\
\hline Wall thickness, $\mu \mathrm{mm}$ & $12.56 \pm 2.59$ & $12.39 \pm 1.96$ & $12.60 \pm 1.83$ \\
\hline Wall cross sectional area, $\mathrm{um}^{2}$ & $4039.6 \pm 1491.0$ & $3943.5 \pm 1093.0$ & $3968.3 \pm 1026.1$ \\
\hline Wall to lumen ratio & $0.29 \pm 0.03$ & $0.28 \pm 0.02$ & $0.28 \pm 0.03$ \\
\hline Basal capiliary density (dorsum), number per area unit & $72.2 \pm 16.4 * *$ & $65.55 \pm 18.6$ & $63.9 \pm 16.7$ \\
\hline Total capillary density (dorsum), number per area unit & $74.2 \pm 14.5$ & $69.6 \pm 20.7$ & $70.0 \pm 11.5$ \\
\hline Basal capillary density (forearm), number per area unit & $56.0 \pm 17.7 \dagger$ & $46.3 \pm 12.8$ & $49.3 \pm 11.6$ \\
\hline Total capillary density (forearm), number per area unit & $57.9 \pm 16.8$ & $53.8 \pm 16.2$ & $53.0 \pm 8.0$ \\
\hline
\end{tabular}

$\times$ TO vs T3: \# TO vs T6: † TO vs T3

\section{EFFECTS OF CHRONIC HEART FAILURE UNCONVENTIONAL THERAPIES ON ENDOTHELIAL FUNCTION}

\author{
C. Tognola ${ }^{1,2}$, I. Cartella ${ }^{1,2}$, M. Milani ${ }^{1,2}$, G. \\ Tavecchia $^{1,2}$, V. Giani ${ }^{1,2}$, M. Biolcati ${ }^{1,2}$, S. Bianchi ${ }^{1,2}$,
}


E. Grasso ${ }^{1,2}$, M. Frigerio $^{3}$, A. Maloberti ${ }^{1,2}$ and C. Giannattasio ${ }^{1,2}$

1 4th Section of Cardiology, DeGasperis CardioCenter, Niguarda Hospital, Milan, Italy; 2 School of Medicine and Surgery, Milan-Bicocca University, Milan, Italy; 3 2nd Section of Cardiology, DeGasperis CardioCenter, Niguarda Hospital, Milan, Italy

INTRODUCTION: Endothelial Dysfunction (ED) of peripheral arteries in Chronic Heart Failure (CHF) subjects has been demonstrated.

AIM: We assessed endothelial function in subjects undergoing unconventional treatments for CHF, namely Heart Transplantation (HTX), continuous-flow Left Ventricular Assist Device implantation (LVAD), and repeated Levosimendan infusions (r-LEVO).

METHODS: Twenty HTX recipients (median time from HTX 21 months), 20 patients supported with LVAD (median time from implant 39 months), and 20 patients receiving monthly Levosimendan infusions (median time on treatment 28 months) were enrolled and compared to a group of 20 healthy subjects. ED was evaluated with ultrasound assessment of the diameter before and after ischemic stress at the brachial artery level. The difference between the two diameters normalized for the baseline value (Flow Mediated Dilation - FMD) has been used for the analysis. All the patients were stable at the time of FMD assessment, with those on r-LEVO being evaluated prior to infusion.

RESULTS: FMD was significantly lower in HTX and LVAD groups with respect to controls $(9.8 \pm 7.4$, $9.3 \pm 5.7$, and $15.6 \pm 6.4 \%$ respectively, $\mathrm{p}=0.01$ ), but not in r-LEVO group $(12.5 \pm 6.9 \%)$. When patients were analyzed according to time from the operation or on treatment, ( $<$ versus $>$ of the median value), no differences were seen in HTX and r-LEVO group, while in LVAD group FMD was borderline significantly higher in patients with longer follow-up $(8.4 \pm 6.4 \%$ versus $10.2 \pm 5.2 \%, \mathrm{p}=0.05)$.

CONCLUSIONS: Based on this preliminary data we can inference the following: 1- FMD is abnormal in HTX recipients, despite their good functional status, probably due to factors unrelated to CHF (e.g. hypertension, renal insufficiency, denervation, and drug effects); 2- LVAD patients also show ED, with possible better adaptation in very long-term survivors; 3 - Near-normal FMD values in CHF patients who remain stable with r-LEVO suggest that pulsed treatment may obtain favourable effects at peripheral level, persisting after clearance of the drug and its metabolites.

\section{RELATIONSHIPS BETWEEN CHOROIDAL THICKNESS AND STATIC AND 24 H INDICES OF AORTIC STIFFNESS IN HYPERTENSIVE PATIENTS}

\author{
G. Mule' (1), C.Carollo (1) E. Mancia (1), \\ K. Montalbano (1), A. Ferotti (1), M. Guarneri (1), \\ D. Altieri (1), S.Cottone (1), M. Vadala'(2)
}

(1) ESH Hypertension Excellence Centre and Nephrology Unit of the University of Palermo, Palermo-Italy; (2)Department of Experimental Biomedicine and Clinical Neuroscience, Section of Ophthalmology, University of Palermo, Palermo, Italy

INTRODUCTION: Although the retina has traditionally been considered the easiest access window for the study of systemic microcirculation, the choroid represents the most vascularized anatomical structure per unit of weight. The option of studying it easily using the new optical coherence tomography (OCT) techniques has made it possible to find out how its thickness is reduced in conditions characterized by high CV risk. This led to hypothesize a potential role of choroidal thickness as a marker of systemic vascular damage.

AIM: to evaluate, in a group of hypertensive patients, the relationships between choroidal thickness and stiffness of the large arteries, a powerful independent predictor of $\mathrm{CV}$ events.

METHODS: 158 hypertensive patients belonging to our Regional Referral Centre for Arterial Hypertension were enrolled. All underwent evaluation of the retino-choroidal district by OCT Swept-Source and oscillometric assessment (BpLab) of the aPWV at rest (static) and during $24 \mathrm{~h}$ blood pressure monitoring (dynamic).

RESULTS: The choroidal thickness of the outer, inner and central rings and the total average of the thicknesses were reduced in subjects with aPWV $>10 \mathrm{~m} / \mathrm{s}$ compared to those who had a lower aortic stiffness, and these differences remained significant even after correction for age and other confounding factors (Figure 9). The average choroidal thickness showed close inverse correlations with the aPWV, both static and dynamic, in the simple linear regression analyses. Among these correlations, that between the static aPWV and the overall average of the choroidal thicknesses $(r=0.498 ; p<0.001)$ is the most significant and remains as such even after correction for various covariates in multiple stepwise regression models. CONCLUSIONS: Our results seem to support the role of choroid thickness as an integrated marker of cardiovascular risk and seem to confirm the theory of a cross-talk between micro- and macrocirculation in hypertensive patients. 
Figure 9.

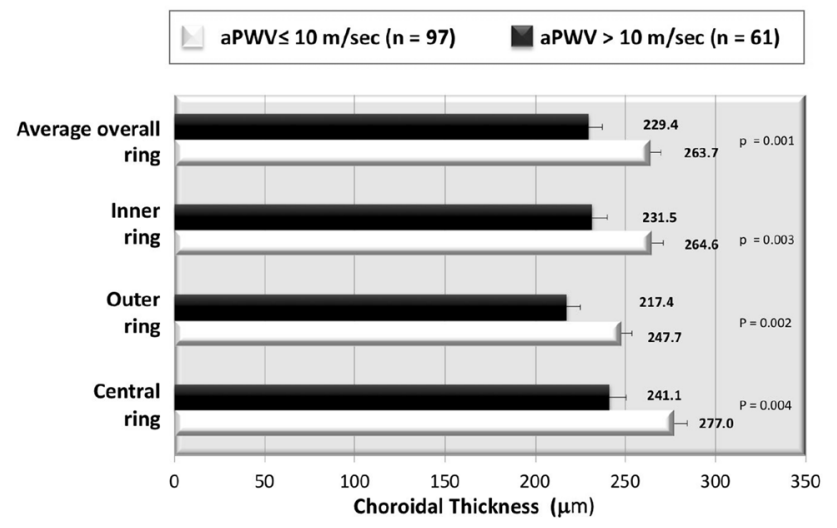

AGE-SPECIFIC, PRESSURE-INDEPENDENT ACUTE CHANGES IN CAROTID-FEMORAL PULSE WAVE VELOCITY DURING HEAD-UP TILT

${ }^{1,2}$ Pucci G., ${ }^{3}$ Avolio A., ${ }^{4,5}$ Spronck B., ${ }^{1}$ Vaudo G.,

${ }^{6}$ Anastasio F., ${ }^{2}$ Van den Meiracker A.H.,

${ }^{2}$ Mattace-Raso F.U.S

1. Department of Medicine, University of Perugia, Unit of Internal Medicine, Terni University Hospital, Terni, Italy; 2. Department of Internal Medicine, Erasmus MC University Medical Center, Rotterdam, The Netherlands; 3. Department of Biomedical Sciences, Faculty of Medicine and Health Sciences, Macquarie University, Sydney, Australia; 4. Department of Biomedical Engineering Yale University, New Haven, CT, United States; 5. Department of Biomedical Engineering, CARIM School

for Cardiovascular Diseases, Maastricht University,

Maastricht, The Netherlands; 6. Unit of Cardiology, ASSTVAL Hospital of Sondrio, Sondrio, Italy

INTRODUCTION: With head-up body tilting, a pressure gradient is generated along the aortic pathway. Aortic stiffness as measured by carotid-femoral pulse wave velocity (cfPWV) is known to depend on blood pressure (BP), and this dependency may change with age. Therefore, the hydrostatic BP gradient resulting from a change in body posture may elicit a cfPWV change that is agedependent.

AIM: to analyze the relationship between BP gradientinduced by head-up body tilting - and related changes in cfPWV in individuals of varying age.

METHODS: cfPWV and other hemodynamic parameters were measured in 30 healthy individuals at head-up tilt of angles of $0^{\circ}$ (supine), $30^{\circ}$, and $60^{\circ}$. At each angle, the PWV gradient and resulting cfPWV were also estimated (predicted) by assuming a global non-linear, exponential, pressure-diameter relationship characterized by a constant $\beta 0$, and taking into account that (diastolic) foot-to-foot cfPWV acutely depends on diastolic BP (DBP).

RESULTS: cfPWV significantly increased upon body tilting $\left(8.0 \pm 2.0 \mathrm{~m} / \mathrm{s}\right.$ supine, $9.1 \pm 2.6 \mathrm{~m} / \mathrm{s}$ at $30^{\circ}$, $9.5 \pm 3.2 \mathrm{~m} / \mathrm{s}$ at $60^{\circ}$, p for trend $\left.<0.01\right)$; a positive trend was also observed for heart rate (HR). When observed, tiltinduced, cfPWV change measured by applanation tonometry was compared with that predicted from the estimated BP hydrostatic gradient, the difference in observed-vs.predicted PWV change increased non-linearly as a function of age ( $\mathrm{R} 2$ for quadratic trend $=0.38, \mathrm{p}<0.01$, $\mathrm{p}$ vs. linear $=0.04)($ Figure 10). This result was unaffected by HR tilt-related variations ( $\mathrm{R} 2$ for quadratic trend $=0.37$, $\mathrm{p}<0.01$, p vs. linear $=0.04)$.

CONCLUSIONS: Under a hydrostatic pressure gradient, the pulse wave traveling along the aorta undergoes agerelated, BP-independent, PWV non-linear increases.

\section{Figure 10.}

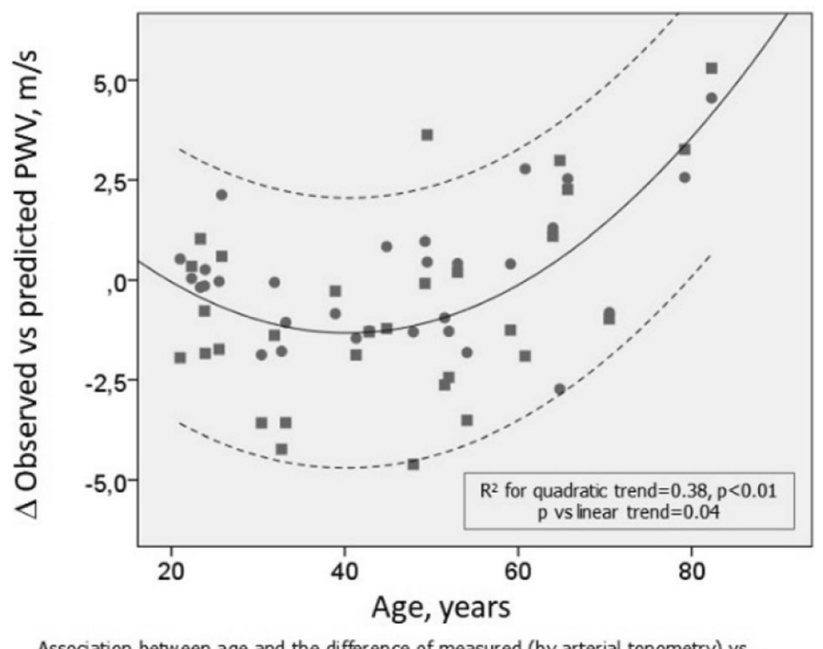

Association between age and the difference of measured (by arterial tonometry) vs. predicted pulse wave velocity $(\mathrm{PWV})$ at each tilt angle $\left(30^{\circ}=\right.$ cirdes, $60^{\circ}=$ squares $)$, expressed as observed-vs.-predicted PWV and adjusted for tilt-related heart rate changes. Solid line: prediction line. Dashed lines: $95 \%$ confidence intervals of the prediction line.

\section{CHANGES IN CAROTID LOCAL STIFFNESS DURING A SIX-YEARS FOLLOW UP IN A GENERAL POPULATION: THE VOBARNO STUDY}

A. Paini, C. Aggiusti, D. Stassaldi, F Bertacchini, S. Capellini, C. Arnoldi, M. Salvetti, M.L. Muiesan

Internal Medicine, University Of Brescia, Italy 
INTRODUCTION: Local carotid stiffness (Carotid PWV) is an independent predictor of cardiovascular events but few data are available on the progression of Carotid stiffness over time.

AIM: The aim of the present longitudinal study was to analyze the progression of local carotid stiffness over a 6-year period in a general population in Northern Italy (Vobarno Study).

METHODS: 123 subjects, age $55 \pm 4$ years $(42 \%$ males, hypertension in $43 \%$ at baseline visit), underwent a baseline (BL) and a follow up (FU) visit, $6.2 \pm 0.5$ years apart. In all subjects laboratory examinations, measurements of blood pressure (BP) and of Carotid PWV (using an echotracking approach) were performed at BL and at FU. Progression rate of Carotid PWV ( $\Delta$ _Carotid_PWV/Year) was defined as (Carotid PWV at FU-Carotid PWV at BL)/ years of FU.

RESULTS: In the overall population, Carotid PWV increased from $6.39 \pm 1.41$ at BL to $6.79 \pm 1.18 \mathrm{~m} / \mathrm{s}$ at FU $(\mathrm{p}<0.05)$. $\Delta$ _Carotid_PWV/Year was inversely related to BP (mean BP: $\mathrm{r}=-0.193, \mathrm{p}<0.05$; pulse pressure: $r=-0.1 .81, p<0.05)$ and to Carotid PWV at BL $(r=-$ $0.669, \mathrm{p}<0.001)$. No other correlation was observed between $\Delta$ _Carotid_PWV/Year and demographic or laboratory parameters at BL. $\Delta$ _Carotid_PWV/Year was also related to changes over time in BP values $(\triangle$ SystolicBP: $\mathrm{r}=0.291, \mathrm{p}<0.01 ; \Delta$ DiastolicBP: $\mathrm{r}=0.266, \mathrm{p}<0.01$; $\Delta$ MeanBP: $r=0.310, p<0.0001)$. At multivariate analysis the variables independently related to the annual progression rate of Carotid PWV were Carotid PWV and mean $\mathrm{BP}$ at BL (beta $-0.68, \mathrm{p}<0.0001$, and beta 0.20 , $\mathrm{p}<0.05$, respectively) and changes in mean BP during follow-up (beta $0.18, \mathrm{p}<0.05$ ).

CONCLUSIONS: In a general population sample in Northern Italy the main determinants of the increase in local carotid stiffness during a 6 years FU were local carotid stiffness and mean BP at BL and change in mean BP over time.

\section{RETINAL WALL TO LUMEN RATIO IN PATIENTS WITH ANGINA AND NO CORONARY ARTERY DISEASE. AN EYE TO THE HEART}

\author{
ML Muiesan ${ }^{1}$, A Paini ${ }^{1}$, C De Ciuceis ${ }^{1}$, C. Agabiti \\ Rosei $^{1}$, V. Guarinoni ${ }^{1}$, C. Rossini ${ }^{1}$, M Salvetti ${ }^{1}, \mathrm{~F}$ \\ Bertacchini $^{1}$, C Aggiusti ${ }^{1}$, D. Rizzoni ${ }^{1}$, R. Giubbini ${ }^{2}, S$ \\ Curello $^{3}, \mathrm{M} \mathrm{Metra}^{3}$ \\ Internal Medicine $e^{1}$, Nuclear Medicine $e^{2} \&$ Cardiology $^{3}$ \\ University of Brescia, Italy
}

INTRODUCTION: The vasculature of the eye and of the heart share several common characteristics. Therefore, the easily accessible vessels of the eye may be considered, to some extent, a "window" to the heart.

AIM: to evaluate retinal arterioles morphological changes in patients with angina and nonobstructive coronary artery disease.

METHODS: In 19 patients (M:W 11:8, mean age $71+6$ years) with microvascular angina (MA), confirmed by normal coronary angiography and SPECT stress hypoperfusion and in 18 controls (C) (M:W 10:8, mean age $69 \pm 6$ years) with normal SPECT response, direct measurement of wall to lumen of retinal arterioles (W/L) using an adaptive optics (AO) imaging system (Imagine Eyes, Orsay, France) was performed. All subjects also underwent BP measurement with an automated oscillometric device (Omron HEM 9000Ai, mean of 3 measurements).

RESULTS: No differences in demographic and hemodynamic characteristics were observed between MA patients and C, except for BMI (BMI $30 \pm 4.2$ vs $24 \pm 4 \mathrm{~kg} / \mathrm{m}^{2}$, in $\mathrm{MA}$ and $\mathrm{C}$ respectively, $\mathrm{p}=0.001)$. Retinal arterioles wall thickness $(11.75 \pm 1.45$ vs $13.9 \pm 1.53 \mu \mathrm{m})$, W/L ratio $(0.29 \pm 0.05$ vs $0.25 \pm 0.03$ in MA and $\mathrm{C}$ respectively, $\mathrm{p}=0.008$ ) and wall cross sectional area (WCSA) (4876 \pm 976 vs $4004 \pm 872 \mu \mathrm{m}^{2}$, respectively in MA and $\mathrm{C}, \mathrm{p}=0.012$ ) were higher in MA patients as compared with C. Differences were confirmed after adjustment for BMI.

CONCLUSIONS: Our findings suggest that in patients with angina and normal coronary arteries retinal structural changes may be observed.

\section{AORTIC STRAIN IMPAIRMENT IN ASCENDING AORTA DILATATION}

Marco Cesareo, Luca Sabia, Eleonora Avenatti, Dario

Leone, Francesco Tosello, Anna Astarita, Giulia

Mingrone, Lorenzo Airale, Franco Veglio, Alberto

Milan

Internal and Hypertension Division, Department

of Medical Sciences, AOU Città della Salute e Scienza, University of Turin, Turin, Italy

INTRODUCTION: Ascending aorta (aA) dilatation is a condition observed in $13 \%$ of hypertensive patients. Little is known about elastic features of aA in hypertensive patients affected by aA dilatation. Aortic strain analysis can describe mechanical properties of aA. The functional evaluation of aA may help in risk assessment and stratification of patients with known aortopathy. 
AIM: To assess mechanical properties of aA by aortic strain in terms of $\beta_{2}$-Stiffness index (Beta-SI) analysis in hypertensive patients with different severity of aA dilatation and to evaluate the association between mechanical properties of aA and cardiovascular damage.

METHODS: 100 hypertensive outpatients underwent clinical evaluation, transthoracic echocardiography (TTE) and assessment of pulse wave velocity (PWV). Strain analysis of aA was performed with speckle-tracking TTE software. Beta-SI was defined as: 100* $\operatorname{Ln}$ (systolic blood pressure/diastolic blood pressure)/maximal transversal deformation of aA. Hypertensive patients were divided in three groups based on aA diameter: $<40 \mathrm{~mm}, 40-45 \mathrm{~mm}$ and $\geq 45 \mathrm{~mm}$.

RESULTS: Beta-SI resulted to rise exponentially with aA dimensions $(\mathrm{p}<0.001)$ with especially in patients having $\mathrm{aA}>45 \mathrm{~mm}$. A progressively greater proportion of patient with impaired (i.e. elevated) Beta-SI was present in groups identified by progressively dilated aA $(18.2 \%$ vs $48.4 \%$ vs $80 \%$ respectively, $\mathrm{p}<0.05)$. On multivariate logistic regression only an impaired Beta-SI predicted aA dilatation $(\mathrm{p}<0.001)$. Beta-SI was also related to cardiovascular organ damage in terms of left ventricular mass (LVMi, $\mathrm{p}=0.030)$ and PWV $(\mathrm{p}=0.028)$. Patients with high BetaSI had grater LVMi $\left(94 \pm 24\right.$ vs. $117 \pm 47 \mathrm{~g} / \mathrm{m}^{2}$; $\mathrm{p}=0.010)$ and PWV $(8.63 \pm 1.88$ vs. $10.20 \pm 2.99 \mathrm{~m} / \mathrm{s}$; $\mathrm{p}=0.013)$.

CONCLUSIONS: A dilatation in hypertensive subjects is associated with increased local aortic stiffness. Strain analysis adds functional information to the mere morphological evaluation of aortic dimension and it could be an useful tool to better stratify cardiovascular risk in this specific population.

\section{Coronavirus and Cardiovascular Risk Factors}

\section{ANTI-COAGULANT THERAPY AND PROGNOSIS IN COVID-19 PATIENTS}

\author{
Boari GEM ${ }^{1}$, Bonetti $\mathrm{S}^{1,2}$, Malerba $\mathrm{P}^{1,2}$, Chiarini $\mathrm{G}^{1,2}$, \\ Braglia Orlandini $F^{1,2}$, Faustini $C^{1,2}$, Bianco $G^{1,2}$, \\ Turini $\mathrm{D}^{1}$, Guarinoni $\mathrm{V}^{1}$, Saottini $\mathrm{M}^{1}$, Pasini $\mathrm{G}^{1}$, Tusi \\ $\mathrm{C}^{1}$. Viola $\mathrm{S}^{1}$, Rizzoni $\mathrm{D}^{1,2}$ \\ 1-Department of General Medicine, Hospital \\ of Montichiari, ASST Spedali Civili Brescia, Montichiari \\ (BS), Italy; 2-Department of Clinical and Experimental \\ Science, University of Brescia, Brescia, Italy
}

AIM: to test the possible relationships between anticoagulant treatment and/or administration of low molecular weight heparin (LMWH) and outcome in a population of 78 COVID 19 patients admitted from 29 February to 31 March 2020 to General Medicine ward of the Hospital Presidium of Montichiari (Bs).

METHODS: A group of 78 patients (54 males and 24 females, aged $69 \pm 13$ years) was included in the present study. In 75 patients, high-flow oxygen was required (Venturi mask), 6 patients needed orotracheal intubation and 10 were given continuous positive pressure oxygen therapy (CPAP). Till June 10th, 17 deaths had been observed. Survival curves were calculated according to Kaplan-Meier method. Comparison between groups was performed with Breslow and Mantel-Cox test.

RESULTS: A significant reduction in survival was observed in patients under treatment with oral anticoagulant drugs (vitamin $\mathrm{K}$ antagonists or new oral anticoagulants) prior to hospital admission (p-0.024), probably as an expression of increased comorbidity and/or more severe cardiovascular disease. On the contrary, the administration of LMWH at a higher dose than usual prophylactic dose (enoxaparin $>4000$ U.C.) was associated with lower mortality (p-0.044, see figure - 12_20200610145550): none of the patients treated died. Administration of LMWH at prophylactic dose was not associated with reduction of fatal events (p-NS).

CONCLUSIONS: The subcutaneous administration of LMWH at a higher dose than that indicated for deep vein thrombosis prophylaxis is associated with a better outcome in patients with SARS-CoV-2 pneumonia, confirming some emerging evidence.

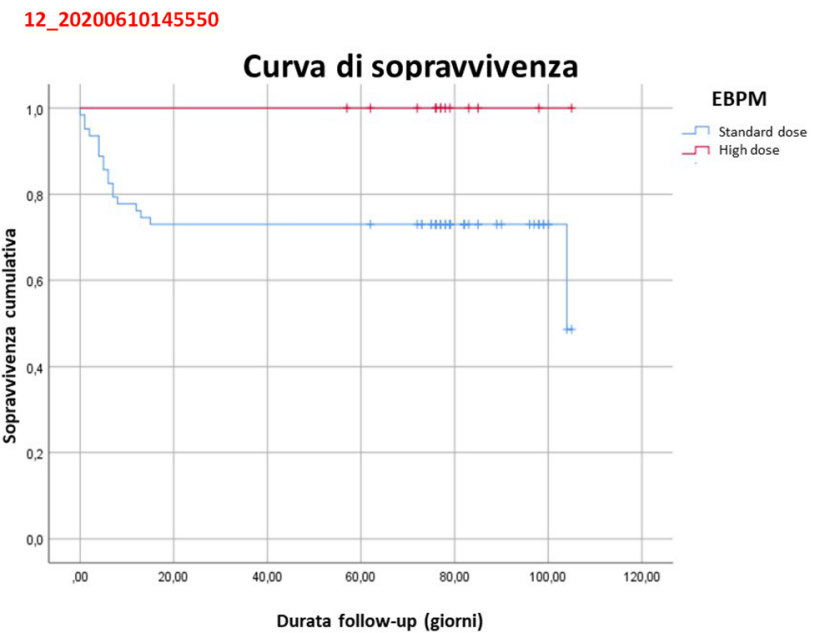




\section{HYPERTENSIVE SUBJECT WITH MULTI-ORGAN DYSFUNCTION DUE TO SARS-CORONAVIRUS-2 INFECTION: SEARCHING FOR A LINK}

Torin Gioia (1), Oliynyk Andriy (2), Casoni Gianluca (3), Aspidistria Laura (3), Viviani Filippo (4), Di Nuzzo Mariachiara (4), Gagliardi Giuseppe (5), Vigna Giovanni (6), Sacco Antonella Paola (1), Rossetti Ciro (1), Zoletto Simone (7), Cuppini Stefano (1), Mazza Alberto (8)

1- Unit of Internal Medicine, S. Maria della Misericordia General Hospital, Rovigo; 2- Public Health School Foundation, Veneto Region, Padova; 3 - Unit of Pulmonology, S. Maria della Misericordia General Hospital, Rovigo; 4 Unit of Infectious Diseases, S. Maria della Misericordia General Hospital, Rovigo; 5 - Unit of Anaesthesia and Resuscitation, S. Maria della Misericordia General Hospital, Rovigo; 6 - Unit of Internal Medicine, S. Luca General Hospital, Trecenta, Rovigo; 7 - Faculty of Medicine and Surgery, University of Padova, Padova; 8 - ESH Excellence Hypertension centre-Unit of Internal Medicine, S. Maria della Misericordia General Hospital, Rovigo, Italy.

INTRODUCTION: Hypertension (HT) seems to increase the severity and the risk of mortality from SARS-Coronavirus-2 (COVID-19 infection). This latter presents few symptoms (fever, cough, diarrhoea), but rarely is aggressive and may lead to interstitial pneumonia and septic shock. A case of COVID-19 infection with multi-organ dysfunction is described.

CASE REPORT: A 53-year-old male was hospitalized in the Infectious Diseases-Unit for fever $\left(38.8^{\circ} \mathrm{C}\right)$, dyspnoea and oxygen (O2) desaturation ( $87 \%$ in ambient air); blood pressure (BP) and heart-rate values were high (148/92 $\mathrm{mmHg}$ and $110 \mathrm{bpm})$. He has a history of HT treated with valsartan $80 \mathrm{mg} /$ daily and hypothyroidism post-thyroidectomy treated with levothyroxine $125 \mathrm{mcg} /$ daily. The nasopharyngeal swab test was positive for COVID-19 virus and the chest CT-scan (Figure - 23_20200604195113) showed lung interstitial disease, bronchiectasis, emphysema and segmental pulmonary embolism of the lower lobes arising from right popliteal venous thrombosis. Despite antibiotic therapy with Piperacillin-Tazobactam $4.5 \mathrm{mg}$ three-a-day and non-invasive C-PAP ventilation with $\mathrm{O} 2$ at $14 \mathrm{~L} / \mathrm{min}$ the patient was transferred to intensive care unit for respiratory distress requiring invasive mechanical ventilation associated with hypotension $(84 / 50$ $\mathrm{mmHg}$ ), renal failure acute, critical illness neuropathy and jaundice. After therapy with sympathomimetic amines, methyl-prednisolone $40 \mathrm{mg}$ /daily, tocilizumab $640 \mathrm{mg}$ for two days, lopinavir/ritonavir $200+50 \mathrm{mg}$ twice-a-day, hydroxychloroquine $200 \mathrm{mg}$ twice-a-day, azithromycin
$500 \mathrm{mg} /$ daily and enoxaparin 8000UI twice-a-day, clinical picture improved as well as blood examinations (leukocytes $16.970 \rightarrow 6.200, \quad$ lymphocytes $1.640 \rightarrow 3.200$, Prothrombin-time $1.34 \rightarrow 1.0$, activated partial thromboplastin-time $45 \rightarrow 29$, D-Dimer $20 \pm 3.68$, Troponin-I $227 \pm 20$, creatinine $1.54 \pm 0.87$, urea $56 \pm 38$, ALT $154 \pm 31$, bilirubin $20 \pm 39$ ). The echocardiogram revealed a basal hypokinesia of the lower wall and of septum suggestive of myocarditis; an abdominal ultrasound documented lithiasic cholecystitis. The patient was discharged after 40 days with BP levels controlled (124/80 $\mathrm{mmHg}$ ) by a fixed-combination therapy with perindoprilamlodipine $5 / 5 \mathrm{mg} /$ daily, anti-thrombotic treatment with DOAC Edoxaban $60 \mathrm{mg} /$ daily and hypolipidemic therapy with atorvastatin $20 \mathrm{mg} /$ daily.

CONCLUSIONS: In HT, the severity of COVID-19 infection seems due to a pro-thrombotic activation of the coagulation cascade rather than the modulation of the renin-angiotensin system. However, further researches investigating the impact of HT and BP lowering drugs on the clinical course of COVID-19 infections are mandatory.

23_20200604195113

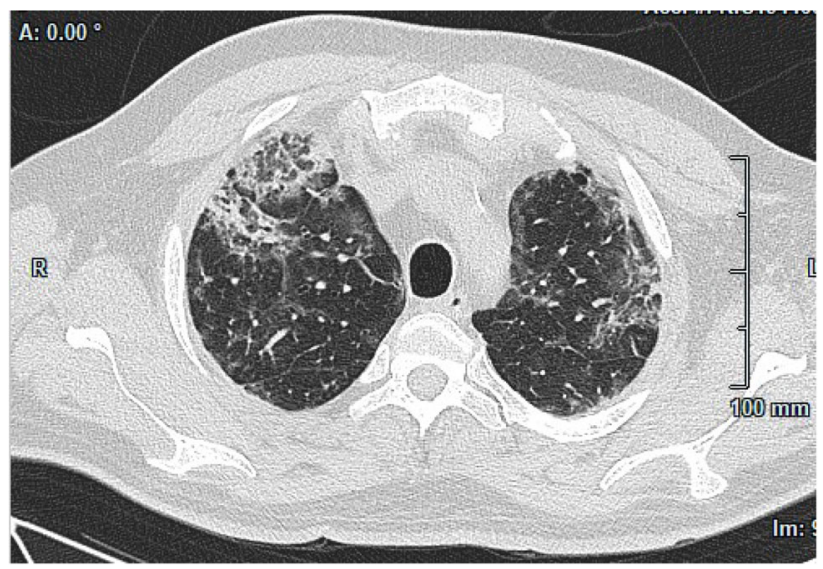

ACE2 AND RHO KINASE INHIBITION IN THE INFECTIOUS/THERAPEUTIC PROCESS OF SARSCOV-2 INFECTION. INSIGHTS FROM A HUMAN MODEL WITH HIGH ACE2 EXPRESSION: GITELMAN'S AND BARTTER'S SYNDROMES

Bertoldi G, Gianesello L, Sgarabotto L, Rigato M, Calò LA

Nephrology, Dialysis and Transplantation Unit, University of Padova 
INTRODUCTION: ACE2, entry point of SARS-CoV-2, has a protective role in SARS-CoV-2-induced Acute Dystress Syndrome (ARDS) and there is a relationship between Rho kinase (ROCK), ARDS and increased ACE2 levels induced by ROCK inhibitors. ACE2 glycosylation, essential for viral binding, is driven by the acidic $\mathrm{pH}$ of intracellular endosomes/lysosomes/Golgi vesicles. The prophylactic/therapeutic use of hydroxychloroquine/ chloroquine finds rationale in its effect of increasing endosomal/Golgi $\mathrm{pH}$, leading to production of non-glycosylated ACE2 and therefore reduction/inhibition of viral binding. Gitelman's and Bartter's syndrome patients (GS/ BS) (rare genetic tubulopathies) have activated RAS, high Ang II yet normotension/hypotension, metabolic alkalosis (not favourable for viral binding), increased and related ACE2 and Ang 1-7 levels, reduced ROCK activity and can provide information on the protective role of increased ACE2 levels and ROCK inhibition.

AIM: This study assesses the impact of COVID-19 on GS/ BS.

METHODS: Telephone survey over 100 GS/BS from Italian hotspots of COVID-19 pandemic (Veneto, Lombardia, Emilia Romagna). COVID-19 prevalence in general population was obtained from Civil Protection's official data, the estimated prevalence from recently published data. 95\% CI values processed with Clopper-Pearson method using R software.

RESULTS: None of GS/BS reported infection/symptoms of COVID-19, which was statistically not significant vs the prevalence of COVID-19 in the general population in the Italian hotspots due to the low prevalence of GS/BS (rare diseases) (95\% CI $0.0-3.0 \%$ vs $0.65 \%, 95 \%$ CI $0.6-0.7 \%$ ) while It becomes significant vs the estimated prevalence in the general population in the hotspots regions $(8.7 \%, 95 \%$ CI $8.7-8.8 \%,(\mathbf{C} 2), \mathrm{p}=0.004)$.

CONCLUSIONS: GS/BS could act as a human model of increased ACE2 for "ex vivo" mechanistic investigation of $\mathrm{CoV}-2$-ACE2 interaction; provide additional evidence on the absence of high ACE2 levels-increased risk of COVID19; demonstrate the non-rationality of the suspension of treatments with ACE inhibitors/sartans, which increase ACE2 expression, to reduce the risk of infection.

\section{EFFECTS OF THE SARS-COV-2 PANDEMIC ON HOSPITALIZATIONS AND CARDIOVASCULAR MORTALITY IN AN ITALIAN PROVINCE WITH LOW PREVALENCE OF COVID-19}

Rita Del Pinto ${ }^{\mathrm{a}}, \mathrm{MD}, \mathrm{PhD}$; Giovambattista Desideri, $\mathrm{MD}^{\mathrm{a}, \mathrm{b}}$; Leondino Mammarella ${ }^{\mathrm{c}}, \mathrm{MD}$; Stefano Abballe ${ }^{\mathrm{a}}$, MD; Sofia Dell'Anna ${ }^{\mathrm{a}}$, MD; Sabrina Cicogna ${ }^{\mathrm{d}}, \mathrm{MD}$;
Davide Grassi ${ }^{\mathrm{a}}$, MD; PhD; Simona $\mathrm{Sacco}^{\mathrm{e}}, \mathrm{MD}$; Claudio Ferri ${ }^{\mathrm{a}}$, MD

${ }^{a}$ University of L'Aquila, Department of Life, Health, and Environmental Sciences, Internal Medicine and Nephrology Unit, San Salvatore Hospital, L'Aquila, Italy; ${ }^{b}$ Geriatrics Unit, Avezzano Hospital, Italy; ${ }^{c}$ Statistics Section, Local Health Authority of the Province of L'Aquila, Italy; ${ }^{d}$ Cardiology and Coronary Care Unit, San Salvatore Hospital, L'Aquila, Italy; ${ }^{e}$ Neurology Section, Department of Applied Clinical Sciences and Biotechnology, University of L'Aquila, Italy

INTRODUCTION: Preliminary data indicate a reduction in hospitalizations for acute coronary syndromes during the Covid-19 outbreak in Northern Italy. The province of L'Aquila, in Central Italy, was marginally affected by the pandemic, but changes in healthcare seeking behaviours were noticed.

AIM: We investigated whether the albeit marginal Covid19 burden in the area of L'Aquila had an impact on cardiovascular hospitalizations and outcomes.

METHODS: We retrospectively analyzed de-identified data from all patients admitted to the five hospitals located in the province of L'Aquila for all-cause and cardiovascular hospitalizations, cardiovascular acute phase treatments (coronary angioplasty, systemic fibrinolysis, endovascular procedures), and in-hospital cardiovascular deaths in the time window from January 1 to March 31, in the years 2020 (study period) and 2019 (control period). Unadjusted incidence rate ratios (IRR) comparing study period and control period for the number of admissions/ procedures were calculated through Poisson regression. Case fatality rate $(\%)$ for cardiovascular diseases in the examined time window was also assessed.

RESULTS: A total of 5908 patients were hospitalized in the first quarter of $2020 \quad(52.9 \%$ women, mean age $59.2 \pm 24.9$ years), with a mean daily admission rate of 64.9. In the same period in 2019 , a total of 6852 patients were hospitalized $(51.8 \%$ women, mean age $60.1 \pm 24.6$ years; 76.1 mean daily admissions; IRR $0.85,95 \%$ CI $0.82-0.88, \mathrm{p}<0.001)$. Significantly less all-cause and cardiovascular hospitalizations occurred in the study period than in the control period (IRR $0.85,95 \% \mathrm{CI} 0.82-0.88$; and IRR $0.73,95 \%$ CI $0.67-0.80$, respectively). The mean daily number of cardiovascular procedures was also significantly lower (N. 219 in 2019, N. 112 in 2020; IRR: 0.74, 95\% CI $0.59-0.93, \mathrm{p}=0.009$ ). Importantly, a disproportionate decrease in the number of procedures was observed in relation to cardiovascular hospitalizations in the study period $(13.8 \%$ versus $19.3 \%, \mathrm{p}=0.001)$. Significantly more intra-hospital cardiovascular deaths occurred in 
March 2020 compared with March 2019 (11.9\% versus $6.1 \%, \mathrm{p}=0.048)$.

CONCLUSIONS: It is possible that late hospital presentations and consequent greater disease severity affected eligibility and outcome of cardiovascular procedures, thus justifying the disproportion in cardiovascular deaths during the national lockdown period. This evidence advances recent concerns regarding the impact of the pandemic on cardiovascular diseases and related deaths.

\section{SUBENDOCARDIAL VIABILITY RATIO IN THE EVALUATION OF MYOCARDIAL DAMAGE IN COVID-19 INFECTION}

\author{
A. Grillo ${ }^{1}$, V. Barbato ${ }^{2}$, V. Bonato ${ }^{2}$, C. Xodo ${ }^{2}$, \\ M. Rovina ${ }^{1}$, N. Colapietro ${ }^{2}$, T. Ferretti ${ }^{2}$, S. Bernardi $^{2}$, \\ M. Bardelli ${ }^{2}$, P. Salvi ${ }^{3}$, B. Fabris ${ }^{2}$

\section{Medicina Clinica, Dipartimento di Medicina, Azienda Sanitaria Universitaria Giuliano Isontina, Trieste; 2 \\ Università di Trieste, Trieste; 3 Dipartimento di Cardiologia, IRCCS Istituto Auxologico Italiano, Milano}

INTRODUCTION: Many patients with COVID-19 show cardiovascular complications, including elevated troponin levels and ischemic electrocardiographic changes, which are associated with a worse overall prognosis. To date, the pathophysiology of these complications is unclear. The Buckberg index or Subendocardial Viability Ratio (SEVR), measured by arterial tonometry and possibly corrected for the blood oxygen content (SEVR-O2) is a useful tool for the evaluation of cardiac ischemia caused by an altered balance between oxygen demand and supply to the subendocardium.

METHODS: Patients admitted to the medical ward for acute COVID-19 infection were evaluated by carotidfemoral arterial tonometry (PulsePen, Diatecne, Milan), arterial blood gas analysis and high sensitivity troponin measurement (hsTnI).

RESULTS: Eight patients (M: F 4: 4) were enrolled, with an average age of $66.7 \pm 15.5$ years, systolic blood pressure $121.5 \pm 10.2 \mathrm{mmHg}$, diastolic $67.6 \pm 10.3 \mathrm{mmHg}$, heart rate $70 \pm 18 \mathrm{bpm}$, arterial oxygen pressure $100 \pm 30$ $\mathrm{mmHg}$ and inspired oxygen fraction $32 \pm 28 \%$; of these, subjects with positive troponin were two (hsTnI peak 227 and $394 \mathrm{ng} / \mathrm{L}$ ). The SEVR showed an average value of $109 \pm 30 \%$ (65-139), which was $19.9 \pm 9.7$ (8.6-34.6) after the correction for oxyhemia (SEVR-O2). In particular, in the two subjects with troponin increase the SEVR was particularly reduced (SEVR 65\% and $81 \%$, SEVR-O2 9.1 and 8.6), confirming a positive correlation between the alteration of the SEVR and myocardial injury.
CONCLUSIONS: Acute COVID-19 infection has high mortality partly mediated by cardiovascular complications. From present data, the increase in troponin, which was found to be a predictor of mortality, is correlated with a significant imbalance between the demand and supply of oxygen to the myocardium. Further and larger studies are needed to confirm the ischemic origin of the rise in troponins and the usefulness of SEVR-O2 as a clinical and prognostic index in patients with COVID-19.

\section{COVID-19 RELATED QUARANTINE EFFECT ON DIETARY HABITS IN A NORTHERN ITALIAN RURAL POPULATION: DATA FROM THE BRISIGHELLA HEART STUDY}

Arrigo F.G. Cicero, Federica Fogacci, Martina Mezzadri, Marina Giovannini, Claudio Borghi on behalf of the Brisighella Heart Study

Hypertension and Atherosclerosis Research Group, Medical and Surgical Sciences Department, University of Bologna, Italy

INTRODUCTION: COVID-19 epidemia has severely hit North-Italy, and this induced the Italian government to adopt severely restrictive measures to reduce the contagion risk, forcing the most part of the population to stop working and leisure activities and to remain at home for several weeks. Beyond the expected decrease in physical activity, it is yet to know how this quarantine affects dietary habits of isolated people.

AIM: To evaluate the effect of COVID-related quarantine on the smoking and dietary habits of a well-characterized northern Italian rural population

METHODS: For the purpose of this study, we selected a subpopulation sample from the database of the Brisighella citizens enrolled in the last population survey to be interviewed on its life-style habit changes during quarantine. The first questions were oriented to exclude those subjects not strictly in quarantine because work-wise active (for instance, farmers or nurses), those respecting quarantine from less than 4 weeks, those affected by COVID-19 infection or by other severe diseases diagnosed after the last population survey, those unable to understand or answer the questions related to the life-style, and those not willing to answer to personal questions by phone. Then, the questionnaire usually administered during the population survey has been telephonically administered to 359 adultelderly subjects by trained personnel (M: 156, W: 203; mean age: $64.6 \pm 13.3$ years old).

RESULTS: Smoking habit were nearly unmodified by the quarantine. Body Mass Index did not modifies during 
quarantine $\left(26.7 \pm 4.7\right.$ vs. $\left.26.7 \pm 4.5 \mathrm{~kg} / \mathrm{m}^{2}\right)$. The selfperceived dietary changes reported by the interviewed subjects are the following ones: no change (50\%), increased quantity (32\%), decreased quantity (6.4\%), selective increase in sweets $(4.2 \%)$, improved quality $(3.4 \%)$, selective increase in charbohydrates $(2.2 \%)$, and decreased quality $(0.9 \%)$. During quarantine, the daily consumption of bread and substitute, pasta and rice, green vegetable and other vegetables, healthy vegetable oils, fruits, milk and yogurt, alcoholic drinks, sugars and sweets, and coffee significantly increased. Among foods classified as never consumed, consumed less than 4 times per week, between 4 and 6 times per weeks, or more than 6 , during quarantine the consumption of lean meat, salamis other than ham, cheeses, eggs, and mixed seed oils significantly increased. On the contrary, the intake of fat meat and ham. Among food classified as never consumed, consumed less than 2 times per week, between 2 and 3 times per weeks, or more than 3 , during quarantine the consumption of fish, mussels, and legumes, significantly decreased, while the one of nuts slightly increased. The Dietary Quality Index decreased from $42.4 \pm 4.1$ to $37.8 \pm 4.7(\mathrm{p}<0.03)$.

CONCLUSIONS: The COVID associated quarantine could worsen the quality of diet, in particular being associated with an increased intake of almost all categories of food.

\section{DETERMINANTS OF HEALING AMONG PATIENTS WITH COVID-19: THE RESULTS OF THE SARS-RAS STUDY OF THE ITALIAN SOCIETY OF HYPERTENSION}

Costantino Mancusi, Guido Grassi, Claudio Borghi, Stefano Carugo, Francesco Fallo, Claudio Ferri, Cristina Giannattasio, Davide Grassi, Claudio Letizia, Pietro Minuz, Stefano Perlini, Giacomo Pucci, Damiano Rizzoni, Riccardo Sarzani, Leonardo Sechi, Franco Veglio, Massimo Volpe, Maria Lorenza Muiesan, Guido Iaccarino, a nome degli investigatori SARS-RAS della SIIA

INTRODUCTION: In the beginning of 2020, an unexpected outbreak due to a new COVID-19 corona virus made the headlines all over the world. Exponential growth in the number of those affected makes this virus such a threat.

AIM: to investigate determinants of healing among COVID-19 patients.

METHODS: We designed a cross-sectional observational multicenter nationwide survey in Italy to understand demographic and clinical characteristics of patients with confirmed COVID-19 infection. We analyzed information from 2446 charts of Italian patients certified for COVID-19 admitted in 26 hospitals. Healing from COVID-19 infection, defined has two negative consecutive swab, was reported in 544 patients $(22.2 \%), 95 \%$ of them were hospitalized.

RESULTS: Age and Charlson Comorbidity Index were significantly lower in patients experienced healing compare to non-healing ( $63 \pm 15$ vs $69 \pm 15$ and $2 \pm 2$ vs $3 \pm 2$ ). Among antihypertensive medications Ace-inhibitors and Diuretic were less frequently prescribed among patients experiencing healing $(\mathrm{p}<0.01)$. In multivariable regression model main predictors of healing were younger age (OR: 0.99; 95\%, 0.98-0.99, $\mathrm{p}=0.0001$ ), being not affected by Chronic kidney disease (OR: 0.35 ; 95\%, 0.17-0.70, $\mathrm{p}=0.003$ ) or heart failure (OR: $0.44 ; 95 \%, 0.28-0.70$, $\mathrm{p}=0.001)$. Non-use ACE inhibitors and diuretics was not significant associated with healing (OR: 0.88; 95\%, $0.67-0.1 .17, \mathrm{p}=0.377$ and OR: $0.77 ; 95 \%, 0.56-1.07$, $\mathrm{p}=0.127$ ).

CONCLUSIONS: Our study demonstrated that age and comorbidities plays a major role in determining healing in patients with COVID-19. Antihypertensive therapy is not associated with less chance of healing.

\section{REMOTE CONTROL OF HYPERTENSIVE PATIENTS DURING COVID-19 PANDEMIC}

Costantino Mancusi, Maria Angela Losi, Raffaela Lombardi, Guido Iaccarino, Giovanni de Simone, Raffaele Izzo, Nicola De Luca on behalf of CSN remote investigators*

University of Napoli Federico II, Napoli, Italy. ${ }^{*}$ CSN remote investigators: Danilo Franco, Ilaria Fucile, Dario Bertolone, Cristina De Colle, Mariangela Mosca, Felice Borrelli, Barbara Fasano, Gaetano Angelotti, Domenico Basile, Maria Rocco

INTRODUCTION: during CODIV 19 pandemic, access to routine medical care was not available due to government restriction. Thus, routine follow-up visit for hypertensive patients was rescheduled and remote control was establish.

AIM: to assess blood pressure (BP) control health status in hypertensive patients from the Campania Salute Network. METHODS: 1110 patients were interviewed using phone call during May 2020. Specific information on BP control, major cardiovascular symptoms and change in BP medications were assessed. In addition, signs and symptoms compatible with COVID-19 infection were inquired.

RESULTS: 722 patients (65\%) answered to the phone call. 642 routinely performed home BP self-measurement. Mean 
BP was $130 \pm 14 / 79 \pm 8 \mathrm{mmHg}$. Uncontrolled home BP $(\geq 135 / 85 \mathrm{mmHg}$ ) was found in $32 \%$ of the patients. During the previous 2 months, $4 \%$ of the patients interrupted antihypertensive therapy, but nobody due to possible interaction with COVID -19 infection. During the last three months 44 patients declare to have symptoms compatible with COVID 19 infection (table 1). 5 patients presented more than 3 symptoms at the same time. 7 patients performed swab and no one had confirmed infection.

\begin{tabular}{|l|l|}
\hline Signs/symptoms & Prevalence (\%) \\
\hline Shortness of breath or difficulty breathing & 6 \\
\hline Dry cough & 11 \\
\hline Fever $>37.5^{\circ} \mathrm{C}$ & 6 \\
\hline Congestion or runny nose/Sore throat & 8 \\
\hline New loss of taste or smell & 2 \\
\hline
\end{tabular}

CONCLUSIONS: During COVID -19 pandemic period, less than $70 \%$ of hypertensive patients had good home BP control. There was no suspension of hypertensive medications due to scaremongering of interaction between ACE inhibitor/ARB and COVID infection. Prevalence of patients with symptoms related to COVID 19 infection was low.

\section{THE RELATIONSHIP BETWEEN MYOCARDIAL INJURY, INFLAMMATION AND COAGULATION ABNORMALITIES IN PREDICTING THE OUTCOME DURING COVID-19}

\author{
Alessandro Mengozzi ${ }^{\mathrm{a}}$, Georgios Georgiopoulos ${ }^{\mathrm{b}, *}$, \\ Agostino Virdis ${ }^{\mathrm{a}^{*}}$, Stefano Masi ${ }^{\mathrm{a},}$, on behalf of Pisa \\ Covid Study Group \\ a. Department of Clinical and Experimental Medicine, \\ University of Pisa, Pisa, Italy; b. School of Biomedical \\ Engineering and Imaging Sciences, King's College \\ London, London, UK
}

INTRODUCTION: High sensitivity troponin $\mathrm{T}$ (hsTnT) elevation is a strong predictor of adverse outcome during SARS-CoV-2 infection but its determinants remain unknown.

AIM: In this study, we assessed the relationship between severity of the inflammatory response/coagulation abnormalities and levels of hsTnT in Coronavirus Disease 2019 (COVID-19). We also evaluated the relevance of these pathways in defining the risk of mortality and complications during hospitalization and the capacity of the treatment to attenuate such a risk.
METHODS: 266 patients hospitalized for SARS-CoV-2 related pneumonia were included in the study. Clinical, biochemical, treatment and outcome data were extracted from the in-hospital medical records. The primary endpoint was incidence of in-hospital COVID-19 associated death. RESULTS: hsTnT was significantly associated with the risk of mortality, even after adjustment for age, creatinine, sex and history of cardiovascular disease. D-dimer and CRP presented stronger associations with hsTnT than $\mathrm{PaO}_{2}$. Changes of hsTnT were associated with changes in the D-dimer and CRP concentrations. Only D-dimer, however, was associated with increased risk of mortality, while CRP was a strong predictor of respiratory complications. Among treatments, only low molecular weight heparin showed convincing evidence for attenuation of the mortality risk in the whole population. This benefit was higher in subjects with higher levels of hsTnT.

CONCLUSIONS: The strong relationship of D-dimer with both hsTnT and mortality, as well as the greater mortality benefits obtained with anticoagulant treatment in people with higher hsTnT suggest that the SARS-CoV-2 coagulopathy might represents an important determinant of the hsTnT elevation and of its relationship with mortality in COVID-19.

\section{Coronavirus and Epidemiology}

\section{INCIDENCE AND DETERMINANTS OF AKI AND ITS IMPACT ON TOTAL MORTALITY IN PATIENTS WITH COVID-19 ADMITTED TO HOSPITAL IN GENOA-NORTHERN ITALY}

Elisa Russo ${ }^{1}$, Pasquale Esposito ${ }^{1}$, Lucia Taramasso ${ }^{2}$, Laura Magnasco ${ }^{2}$, Michela Saio ${ }^{1}$, Federica Briano ${ }^{2}$, Chiara Russo $^{2}$, Antonio Vena ${ }^{2}$, Antonio Di Biagio ${ }^{2}$, Matteo Bassetti ${ }^{2}$, Francesca Viazzi ${ }^{1}$

${ }^{1}$ Department of Internal Medicine, Clinica Nefrologica Dialisi e Trapianto, University of Genoa, Hospital Policlinico San Martino - IRCCS, Genoa, Italy; ${ }^{2}$ Infectious Diseases Clinic, Department of Health Sciences, University of Genoa, Hospital Policlinico San Martino - IRCCS, Genoa, Italy

INTRODUCTION: The prevalence of kidney involvement during SARS-CoV-2 infection has been reported to be high in several clinical records around the world. Nevertheless, depending by study population, definitions of kidney involvement and length of observation, the pictures described are inconsistent.

METHODS: We collected data on patient demographics, baseline history of comorbid conditions, home 
medications, details about triage vitals, baseline laboratory test results and in-hospital treatment in the first 854 patients who received a diagnosis of COVID-19 consecutively admitted to our Institution in Genoa-north of Italy. The diagnosis of chronic kidney disease (CKD) was based on the median serum creatinine and on the presence of proteinuria at urinalysis when available within 180 days prior to hospital admission. Acute kidney injury (AKI) was defined according to Kidney Disease: Improving Global Outcomes (KDIGO) criteria. The primary and secondary outcome were the development of AKI and death.

RESULTS: Of 777 patients admitted with Covid-19 and with available kidney data within 6 months before, AKI developed in 176 patients (22.6\%). Of these, 79 (45\%) showed an acute worsening of a pre-existing CKD, and $12.6 \%$ required kidney replacement therapy. Independent associates of AKI were CKD, PCR, the need for ventilation. Among patients with AKI, 111 died (63\%) and its occurrence increased by $60 \%$ the risk for death (HR 1.60 [95\% IC $1.21-2.49$ ] $\mathrm{p}=0.002$ ) independently by potential confounding factors including hypertension, pre-existing kidney damage, and comorbidity index. Treatment with HCQ was associated with a decreased risk of AKI and death (by 60 and 50\%, respectively). Patients with kidney damage showed a similar distribution of the causes of death as compared to the whole population except for bleeding who was significantly more frequent in AKI as compared to $\mathrm{CKD}$ and the whole population (7.5 vs 1.5 vs $3.5 \%$, respectively).

CONCLUSIONS: Awareness of kidney status, both preexisting CKD and the eventual development of AKI, may help to choose a more adequate therapeutic strategy improving the vital prognosis of patients with COVID-19.

\section{CORONAVIRUS-19 DISEASE (COVID-19) IMPACT ON THE ADMISSION IN EMERGENCY DEPARTMENT FOR CARDIOVASCULAR DISEASE}

\author{
Lucilla Crudele $^{1,2}$, Roberta Buono ${ }^{1,2}$, Sebastiano \\ Cicco $^{1,3,6}$, Rocco Guerra ${ }^{4,5}$, Vito Racanelli ${ }^{1}$, Angelo \\ Vacca $^{1}$, and Giuseppe Ranieri ${ }^{1,6}$
}

1. Unit of Internal Medicine "Guido Baccelli", Department of Biomedical Sciences and Human Oncology, University of Bari Aldo Moro Medical School, Bari (BA), Italy; 2. Emergency Department, AUOC Policlinico di Bari, Bari, Italy; 3. Emergency Department, "Don Tonino Bello" Hospital, ASL Bari, Molfetta (BA), Italy; 4. Unit of Public
Health, Department of Biomedical Sciences and Human Oncology, University of Bari Aldo Moro Medical School, Bari, Italy; 5. Health Management Department, "Umberto I" Hospital, ASL Bari, Corato (BA), Italy; 6. Centro Ipertensione, Unit of Internal Medicine "Guido Baccelli", Department of Biomedical Sciences and Human Oncology, University of Bari Aldo Moro Medical School, Bari (BA), Italy;

INTRODUCTION: Emergency Departments (ED) are the front-line setting in fighting the infection by the new coronavirus SARS-CoV-2. For the purpose of reducing SARS-CoV-2 infectiousness, whole nation lockdown was declared since March 11th, 2020. The main rule was to stay at home and not to go to ED if it was not mandatory. In Apulia, Health care was organized in 9 COVID hospitals with dedicated ED, while the remaining hospitals were selected to treat other diseases.

AIM: We performed a study on ED to check patients in three non-COVID ED compared to COVID ED. Together these hospitals take care of more than 1,000,000 residents. We compared discharges between March 11th and April 10th, 2020 to the same interval in 2019 and 2018.

METHODS: Using ICD9 discharge diagnosis code, we focused our preliminary analysis on cardiovascular diseases: ischemic cardiac pain, both as myocardial infarction and ischemic angina, Stroke, Heart failure, Cardiac Arrest, Hypertension, and Cardiac Arrhythmias. We also analyzed data on non-cardiac chest pain and Anxiety. All patients were SARS-CoV-2 negative at reverse transcriptionpolymerase chain reaction analysis on the swab sample. All listed diagnosis were related to the population cared. Variables are compared using Pearson's Chi square test. A p value $<0.05$ was considered statistically significant.

RESULTS: We found out that during 2020 lockdown the global number of patients admitted to ED significantly decreased compared to the same period of two previous years. Discharges in 2020 were significantly reduced for ischemic disease, stroke, hypertension and cardiac arrhythmias. In addition, non-cardiac chest pain and anxiety were significantly reduced in 2020 . On the contrary, heart failure was stable during years since no significant difference in 2020 compared to 2019 and 2018 were seen. We also found a significant increase in cardiac arrests in 2020 compared to 2018 and 2019. These results are the same also splitting COVID and non-COVID EDs data.

CONCLUSIONS: The difference in the 2020 diagnoses may also imply a consequence for the future. Differently to past successes, after COVID-19 a possible increase in chronic consequence like heart failure or stroke sequelae 
may result. Health system should address events that may be regarded as another consequence of COVID-19 pandemic.

\section{Coronavirus Infection}

\section{RELATIONSHIP BETWEEN HYPERTENSION, ANTI-HYPERTENSIVE THERAPY AND PROGNOSIS IN COVID-19 PATIENTS}

Boari GEM ${ }^{1}$, Chiarini $\mathrm{G}^{1,2}$, Bonetti $\mathrm{S}^{1,2}$, Malerba $\mathrm{P}^{1,2}$, Braglia Orlandini $F^{1,2}$, Bianco $G^{1,2}$, Faustini $C^{1,2}$, Turini $\mathrm{D}^{1}$, Saottini $\mathrm{M}^{1}$, Guarinoni $\mathrm{V}^{1}$, Viola $\mathrm{S}^{1}$, Desenzani $\mathrm{P}^{1}$, Bonzi $\mathrm{B}^{1}$, Nardin $\mathrm{M}^{1,2}$, Rizzoni $\mathrm{D}^{1,2}$

1-Department of General Medicine, Hospital of Montichiari, ASST Spedali Civili Brescia, Montichiari (BS), Italy; 2-Department of Clinical and Experimental Science, University of Brescia, Brescia, Italy

INTRODUCTION: It has been suggested that hypertension and anti-hypertensive therapy may influence prognosis in patients affected by SARS-CoV-2 pneumonia.

AIM: to evaluate possible relationship between hypertension, ongoing anti-hypertensive treatment and mortality in a population of 78 patients admitted from 29 February to 31 March 2020 to General Medicine ward of the Hospital of Montichiari (Bs).

METHODS: A group of 78 patients (54 males and 24 females, aged $69 \pm 13$ years) was included in the present study. In 75 patients, high-flow oxygen was required (Venturi mask), 6 patients needed orotracheal intubation and 10 were given continuous positive pressure oxygen therapy (CPAP). As of June 10th, 17 deaths had been observed. Survival curves were calculated according to Kaplan-Meier method. Comparison between groups was performed with Breslow and Mantel-Cox test

RESULTS: A significant raise in mortality was observed in hypertensive patients $(\mathrm{p}=0.008$ - see figure 12_20200610140123) at standard follow-up (from admission to 10th June); the result was confirmed even after reducing follow-up period to hospitalization time. Ongoing therapy with ACE-inhibitors at the time of admission was associated with a worse prognosis $(p=0.083)$, while no difference in survival was observed in patients under treatment with ARBs $(p=0.68)$, statins $(p=0.33)$ or antiplatelets $(\mathrm{p}=0.095)$. A trend to significance was detected between COPD and mortality $(\mathrm{p}=0.059)$. Administration during hospitalization of steroids, anti-viral drugs, antimalaric drugs or antibiotics (azithromycin and others) didn't impact on prognosis.
CONCLUSIONS: Hypertension is a negative prognostic factor in patients affected by SARS-CoV-2 pneumonia. This confirms previous observations, although some concerns about this point still remain.

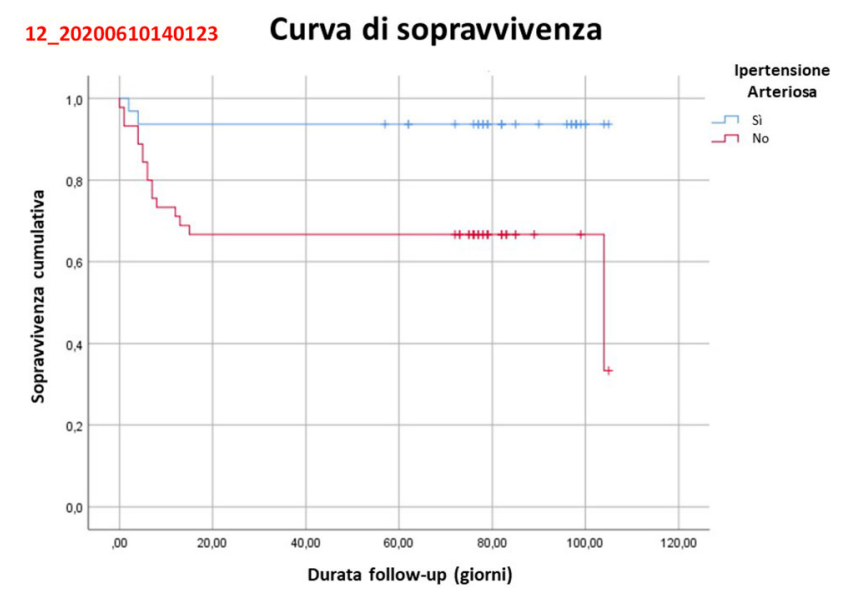

\section{HOME BLOOD PRESSURE DURING CORONAVIRUS PANDEMIC PHASE 1: PHONE INTERVIEWS AND WELL-BEING}

\author{
P. Nazzaro, A. Nardecchia, M. Contini, G. Schirosi, \\ L. Debenedittis, G. Aceto, G. Laselva, I. Vitali, \\ G. Servedio, G. Polieri, M. Biallo
}

Dept of Neurosciences, Unit of Hypertension "AM. Pirrelli", "Aldo Moro" University of Bari, Italy

INTRODUCTION: Different studies highlighted the association between home blood pressure (HBP), compliance to the treatment and hypertensive state but in very few the individual wellbeing could be detected during social stress conditions.

AIM: to verify these associations with the vascular damage markers previously found 10-14 months before the Covid 19 pandemic phase 1 .

METHODS. Between march and april 2020, 730 grade-1 hypertesives, with similar age, hypertensive state and treatment, were interviewed by phone. By adapted SF-36 questionnaire, the emotional (EMO) and physical (PHY) state, the quality of life ascribed to the hypertensive state (HTNqol) and treatment (TRATqol) were investigated. The SBP/DBP difference between last HBP and medical visit (dom-off), the frequency (FREQ) of BP measurements, the previously ascertained arterial stiffness (PWVcf), central SBP and cognitive impairment (COGtot) were also considered. 
RESULTS: Patients, divided in 425 with lower $(\mathrm{HBP}<$ ) and 305 with higher $(\mathrm{HBP}>$ ) than $135 / 85 \mathrm{mmHg}$, did not differ for SBP/DBPoff $(132 \pm 14 / 78 \pm 10$ vs $133 \pm 16 /$ $79 \pm 9$ ), compliance, weight gain, quality/time of sleep, risk of sleep apnoea and smoke habit, but for SBP/DBPhbp $(123 \pm 7 / 76 \pm 8$ vs $143 \pm 9 / 81 \pm 8, \mathrm{p}<0,001)$ and other characteristics. $\quad(\mathrm{m} \pm$ s.d.; $\quad *: \mathrm{p}<.05, \quad * *: \mathrm{p}<.01$, $* * *: \mathrm{p}<.001$ vs $\mathrm{HBP}<$ ).

\begin{tabular}{|l|l|l|l|l|l|l|}
\hline $\mathrm{pz} /$ var & SBP/DBPdom-off & FREQ & SBPcar & PWVcf & EMO & PHY \\
\hline HBP $<$ & $-8 \pm 18 / 2 \pm 12$ & $19 \pm 26$ & $116 \pm 12$ & $8.8 \pm 4.1$ & $19.1 \pm 3.5$ & $10.1 \pm 1.9$ \\
\hline HBP $>$ & $8 \pm 16 / 1 \pm 13^{* * *}$ & $36 \pm 73^{* * *}$ & $121 \pm 14^{* * *}$ & $10.5 \pm 2.6^{* * *}$ & $17.2 \pm 2.9^{* * *}$ & $9.4 \pm 1.8^{* * *}$ \\
\hline
\end{tabular}

COGtot $(24.8 \pm 7.8$ vs $29.3 \pm 8.4 * * *)$, HTNqol $(4.5 \pm 0.9$ vs $3.9 \pm 0.9 * * *)$ e TREATqol $(4.6 \pm 0.8$ vs $\left.4.1 \pm 0.9^{* * *}\right)$ were impaired in $\mathrm{HBP}>$ patients. The Pearson test showed the significant association between SBPdom-off and SBPcar $\left(0.513^{* * *}\right)$ and PWVcf $\left(0.272^{* *}\right)$.

CONCLUSIONS: The findings demonstrate that the wellbeing in hypertensives is associated to compliance, preserved vascular function and cognitive capabilities also during social stress. These variables should be considered along the follow-up of hypertensives to enhance the efficacy of treatment since early phases of the disease.

\section{RELATIONSHIP BETWEEN BLOOD PRESSURE BEHAVIOR, RAAS-I TREATMENT AND TOTAL MORTALITY IN PATIENTS WITH COVID-19 ADMITTED TO HOSPITAL IN GENOA- NORTHERN ITALY}

Elisa Russo ${ }^{1}$, Pasquale Esposito ${ }^{1}$, Lucia Taramasso ${ }^{2}$, Laura Magnasco ${ }^{2}$, Michela Saio ${ }^{1}$, Federica Briano ${ }^{2}$, Chiara Russo ${ }^{2}$, Antonio Vena ${ }^{2}$, Antonio Di Biagio ${ }^{2}$, Matteo Bassetti ${ }^{2}$, Francesca Viazzi ${ }^{1}$

\section{${ }^{1}$ Department of Internal Medicine, Clinica Nefrologica Dialisi e Trapianto, University of Genoa, Hospital Policlinico San Martino - IRCCS, Genoa, Italy; ${ }^{2}$ Infectious Diseases Clinic, Department of Health Sciences, University of Genoa, Hospital Policlinico San Martino - IRCCS, Genoa, Italy}

INTRODUCTION: Hypertension (HT) is a major risk factor of mortality and its impact in patients affected by SARS-COV-2 is further emphasized worldwide. RAAS imbalance hypothesis has been involved in the explanation of the mortality rates of compromised patients. Nevertheless, a better understanding of the relationship between blood pressure (BP) behaviour, RAAS-inhibition (RAAS-I) and death in patients affected by COVID-19 is needed.

METHODS: We collected data on patient demographics, baseline history of comorbid conditions, home medications, details about triage vitals, baseline laboratory test results and in-hospital treatment in the first 854 patients consecutively admitted to our Institution in Genoa-Northern Italy who received a diagnosis of COVID-19. Systolic blood pressure (SBP) tertiles were defined as $<120$, $120-139$ and $\geq 140 \mathrm{mmHg}$. The primary endpoint was allcause mortality.

RESULTS: Among 750 patients with all information available, 371 patients (49\%) had a history of hypertension and $266(35 \%)$ died during a $36 \pm 22$ days of follow-up. The presence of HT increased by $40 \%$ the risk for death (HR 1.43 [95\% IC 1.40-.30] $\mathrm{p}<0.0001$ ) independently by potential confounding factors including age, gender, diabetes, pre-existing kidney damage, the need for invasive ventilation and treatment with ACE-Inhibitors or Angiotensin receptor blockers. When we analysed the whole study population on the basis of BP tertiles we found a $\mathrm{j}$-shaped relationship between BP values and the occurrence of acute kidney injury and of all cause death. Patients with BP values between 120 and $140 \mathrm{mmHg}$ showed a significantly lower risk for death as compared to those with $\mathrm{BP}$ values above 140 or below $120 \mathrm{mmHg}$. This finding is shown as Kaplan-Maier plot (Log rank test $\mathrm{p}<0.0001)$.

CONCLUSIONS: HT entails a worse prognosis in COVID-19. RAAS-I is not associated with a different severity in outcome. The relationship between BP values, renal function and death seems to be J-shaped, with optimal SBP values between 120 and $140 \mathrm{mmHg}$.

\section{Coronavirus and Antihypertensive Therapy}

\section{LUNG DAMAGE IN SEVERE-ACUTE- RESPIRATORY-SYNDROME-CORONAVIRUS-2 (SARS-COV-2) INFECTION: THE RENIN- ANGIOTENSIN-SYSTEM IMBALANCE DUE TO A REDUCED ACTIVITY OF ANGIOTENSIN- CONVERTING-ENZYME-2 (ACE2) IS LIKELY TO PLAY A KEY ROLE}

Riccardo Sarzani ${ }^{1,2}$, Federico Giulietti ${ }^{1,2}$, Chiara Di Pentima $^{1}$, Piero Giordano ${ }^{2}$, Francesco Spannella ${ }^{1,2}$

1) Department of Clinical and Molecular Sciences, University "Politecnica delle Marche", Via Tronto 10/a, Ancona, Italy; 2) Internal Medicine and Geriatrics, IRCCS INRCA, Via della Montagnola 81, Ancona, Italy

INTRODUCTION: An imbalance of the Renin-Angiotensin-System (RAS) is likely to play a key role in the genesis of both lung damage and the consequent acute respiratory distress syndrome (ARDS) found in viral 
pneumonia such as that caused by the Severe-Acute-Respiratory-Syndrome-Coronavirus-2 (SARS-CoV-2) infection (COVID-19).

METHODS: We performed an accurate revision of the available literature regarding both preclinical and clinical studies on the association between RAS imbalance and the onset of acute lung damage, which is the main clinical feature of COVID-19, assuming a possible beneficial role of RAS-modulating drugs.

RESULTS: The respiratory symptoms of COVID-19 are caused by the SARS-CoV-2 infection of the pneumocytes, with subsequent involvement of both alveolar interstitium and vascular endothelial cells, mediated by the binding of the virus to its receptor: the Angiotensin-ConvertingEnzyme-2 (ACE2). The binding of the viral spike protein to ACE2 leads to its down-regulation. ACE2 plays a key role in preserving the equilibrium of the two main RAS arms: the ACE/angiotensin (Ang) II/Ang II type 1 receptor (AT1R) axis ("classic RAS") and the ACE2/Ang 1-7/ MasR axis ("anti-RAS"). Following the down-regulation of ACE2 due to the viral binding, there is an hyperactivation of the "classic RAS", with attenuation of the lung and cardiovascular protective roles of the "antiRAS", resulting in endothelial disfunction and microvascular damage with increased permeability, leaky vessels and secondary release of proinflammatory cytokines, leading to alveolar epithelium dysruption and fibrosis. The evidence of a more aggressive COVID-19 evolution in obese males, in whom the "classic RAS" is already hyperactivated due to adiposity, support this hypothesis. Both AT1R-blockers (ARB) and ACE-inhibitors (ACEi) are likely to reduce both lung damage and fibrosis by modulating the activity of the "classic RAS" and inducing ACE2, thus rebalancing the two opposing arms. Indeed, several animal models and preclinical studies support this hypothesis.

CONCLUSIONS: Clinical randomized controlled trials are needed to confirm the protective role of RAS-modulating drugs, such as ARB and ACEi, on lung and cardiovascular damage caused by SARS-CoV-2 infection, that are supported by strong pathophysiological basis and both preclinical and clinical evidences. 